\title{
Fission Product Separation from Pyrochemical Electrolyte by Cold Finger Melt Crystallization
}

Joshua R. Versey

August 2013

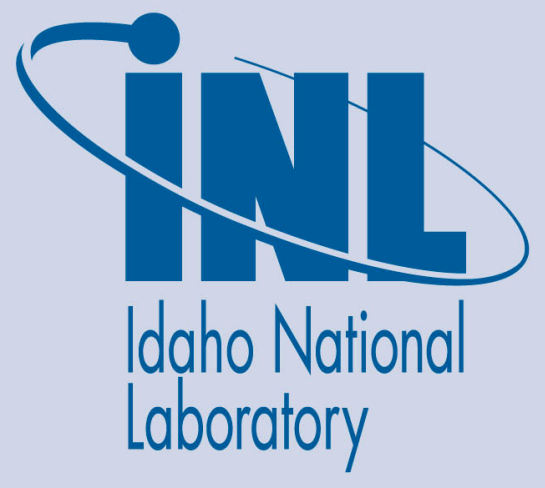

The INL is a U.S. Department of Energy National Laboratory operated by Battelle Energy Alliance 
INL/EXT-13-30068

\title{
Fission Product Separation from Pyrochemical Electrolyte by Cold Finger Melt Crystallization
}

${ }^{1}$ University of Idaho

\author{
Joshua R. Versey ${ }^{1}$
}

August 2013

\begin{abstract}
Idaho National Laboratory
Idaho Falls, Idaho 83415
\end{abstract}

http://www.inl.gov

Prepared for the

U.S. Department of Energy

Through the INL LDRD Program

Under DOE Idaho Operations Office

Contract DE-AC07-05ID14517 
FISSION PRODUCT SEPARATION FROM PYROCHEMICAL ELECTROLYTE BY COLD FINGER MELT CRYSTALLIZATION

\author{
A Thesis \\ Presented in Partial Fulfillment of the Requirements for the \\ Degree of Master of Science \\ with a \\ Major in Nuclear Engineering \\ in the \\ College of Graduate Studies \\ University of Idaho \\ by \\ Joshua R. Versey
}

August 2013

Major Professor: Supathorn Phongikaroon, Ph.D. 


\section{Authorization to Submit Thesis}

This thesis of Joshua R. Versey, submitted for the degree of Master of Science with a major in Nuclear Engineering and titled "Fission Product Separation from Pyrochemical Electrolyte by Cold Finger Melt Crystallization," has been reviewed in final form. Permission, as indicated by the signatures and dates given below, is now granted to submit final copies to the College of Graduate Studies for approval.

Major Professor

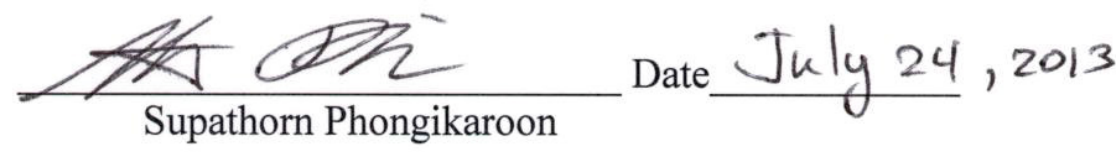

Committee Members

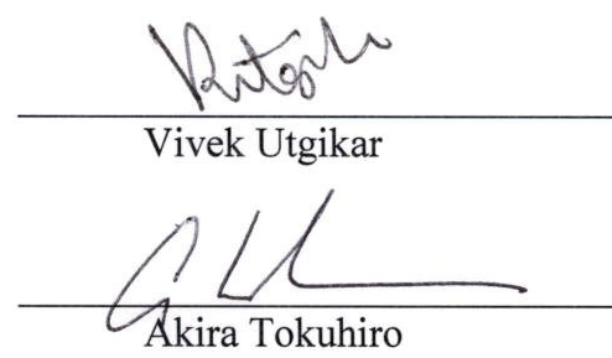

Date July 24,2013

Department

Administrator

Discipline's

College Dean

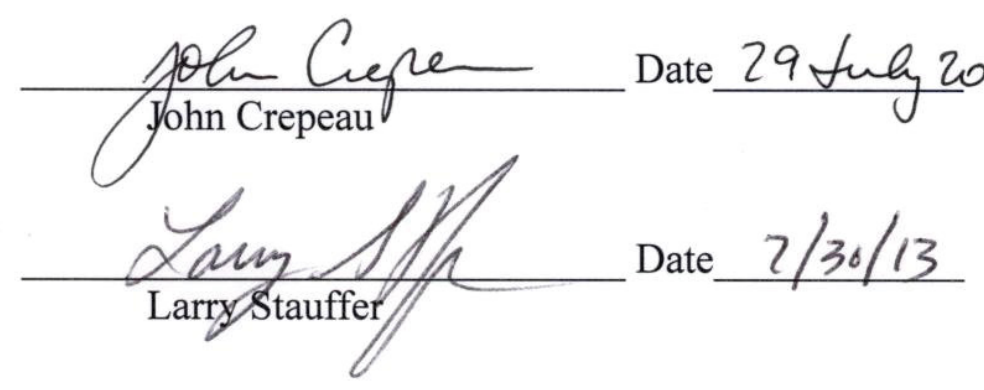

Final Approval and Acceptance by the College of Graduate Studies

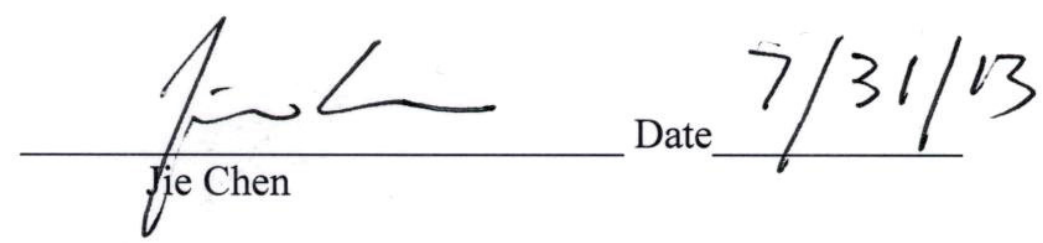




\begin{abstract}
This work contributes to the development of pyroprocessing technology as an economically viable means of separating used nuclear fuel from fission products and cladding materials. Electrolytic oxide reduction is used as a head-end step before electrorefining to reduce oxide fuel to metallic form. The electrolytic medium used in this technique is molten $\mathrm{LiCl}-\mathrm{Li}_{2} \mathrm{O}$. Groups I and II fission products, such as cesium (Cs) and strontium ( $\mathrm{Sr}$ ), have been shown to partition from the fuel into the molten $\mathrm{LiCl}-\mathrm{Li}_{2} \mathrm{O}$. Various approaches of separating these fission products from the salt have been investigated by different research groups. One promising approach is based on a layer crystallization method studied at the Korea Atomic Energy Research Institute (KAERI). Despite successful demonstration of this basic approach, there are questions that remain, especially concerning the development of economical and scalable operating parameters based on a comprehensive understanding of heat and mass transfer. This research explores these parameters through a series of experiments in which $\mathrm{LiCl}$ is purified, by concentrating $\mathrm{CsCl}$ in a liquid phase as purified $\mathrm{LiCl}$ is crystallized and removed via an argon-cooled cold finger. All experiments were conducted in an inert argon atmosphere. The experimental $\mathrm{LiCl}-\mathrm{CsCl}$ operating temperature was $650^{\circ} \mathrm{C}$ with a cold finger temperature held at the freezing temperature of $\mathrm{LiCl}\left(\approx 605^{\circ} \mathrm{C}\right)$. Molten salt and cold finger exhaust temperatures were measured throughout experiments and argon cooling gas flow was controlled manually via a needle valve. The varied parameters of interest were initial contaminant concentration, cold finger cooling rate, and separation time. $\mathrm{CsCl}$ concentration was measured via Inductively Coupled Plasma Mass Spectroscopy (ICP-MS) for purified LiCl crystals and concentrated LiCl-CsCl bulk. Preliminary results revealed that co-deposition of $\mathrm{CsCl}$ along with $\mathrm{LiCl}$ crystal formation due to slightly non-equilibrium mass transport is a problem that must be minimized. Analysis of experiments with initial $5 \mathrm{wt} \% \mathrm{CsCl}$ concentration showed that for an experimental matrix of varying cold finger coolant flow rates $(7.4,9.8,12.3$, and $14.9 \mathrm{~L} / \mathrm{min})$ and separation times $(5,10,15$, and $30 \mathrm{~min})$, the optimal cooling flow rate and separation time are $14.9 \mathrm{~L} / \mathrm{min}$ and $15 \mathrm{~min}$, respectively, producing a $0.33 \mathrm{wt} \% \mathrm{CsCl}$ crystal purity at a production rate of 0.36 $\mathrm{g} / \mathrm{min}$. Experimental results for initial concentrations of 1,3 , and $7.5 \mathrm{wt} \% \mathrm{CsCl}$ at the aforementioned coolant flow rates for separation times of 15 min were analyzed and showed
\end{abstract}


that as initial bulk concentration increased from 1 to $7.5 \mathrm{wt} \% \mathrm{CsCl}$, the change in purified crystal concentration for experiments was less than $0.5 \mathrm{wt} \% \mathrm{CsCl}$ for all but one data point. The optimal result of the initial $5 \mathrm{wt} \% \mathrm{CsCl}$ experiments along with results of initial 1, 3, and $7.5 \mathrm{wt} \% \mathrm{CsCl}$ bulk concentration experiments were used to predict a scale-up scenario that provided a potential separation rate and purity of $136 \mathrm{~g} / \mathrm{hr}$ and $0.24 \mathrm{wt} \% \mathrm{CsCl}$, respectively, to provide insight that will aid in future economic and feasibility analysis of cold finger separation as a commercial separation process.

Six crystal growth rate correlations were developed to better understand crystal growth rate behavior and determine the rate controlling physics of the cold finger melt crystallization separation process. The first three correlations accounting for heat, mass, and heat $\&$ mass transfers were proposed with the use of dimensionless Grashof, Prandtl, and Schmidt numbers. The correlation took the form of a conventional Nusselt or Sherwood correlation for a natural convective system. The correlation accounting for both heat and mass transfer in the molten salt system had the best fit to the experimental data sets with an $R^{2}$ value of 0.87 . The fitted results indicated that the dimensionless parameter accounting for mass transfer $(S c)$ had a larger contribution to the magnitude of the independent variable of this correlation than the dimensionless parameter accounting for heat transfer $(\mathrm{Pr})$. Analysis of the first three correlations also revealed that they predict experimental dimensionless crystal masses well for slower cooling gas flow rates and not as well for faster cooling rates. This is due to larger deviations from phase equilibrium temperatures during the separation process for faster cooling rates. Three additional correlations were proposed to elucidate the behavior for the components of the independent variables of the first three correlations using the experimental data performed with initial 1,3, and $7.5 \mathrm{wt} \% \mathrm{CsCl}$, and the new correlations were used to predict the rest of the initial $5 \mathrm{wt} \% \mathrm{CsCl}$ experimental data not used to curve fit the new correlations. The three new correlations provided predictions of the experimental data performed at initial $5 \mathrm{wt} \% \mathrm{CsCl}$ with an average percent change root mean square deviation $(R M S D)$ of 22.1 and $R^{2}$ of 0.848 . The overall analysis has shown the strong controlling effect that cooling gas flow rate has on crystal growth rate compared to initial $\mathrm{CsCl}$ concentration and crystal growth time. 


\section{Acknowledgments}

This work was made possible by the Laboratory Directed Research and Development (LDRD) Program of Idaho National Laboratory, administered by the Center for Advanced Energy Studies, under the Department of Energy Idaho Operations Office Contract DEAC07-05ID14517. 


\section{Table of Contents}

Authorization to Submit Thesis ........................................................................................... ii

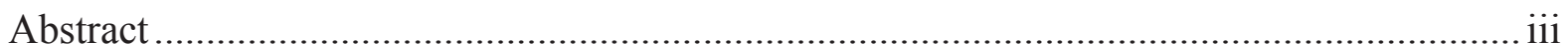

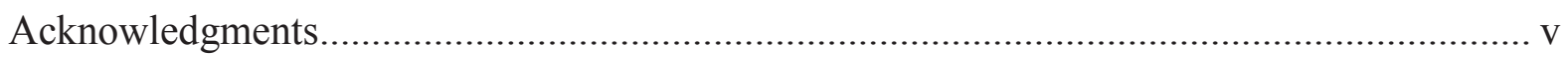

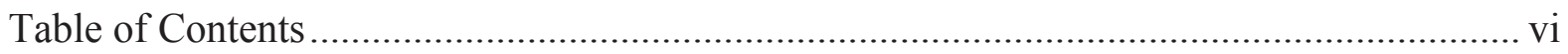

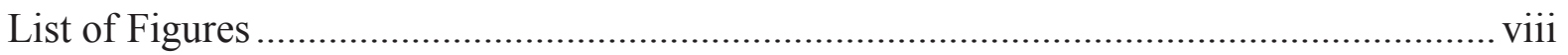

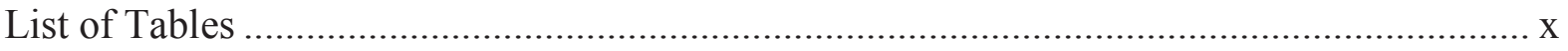

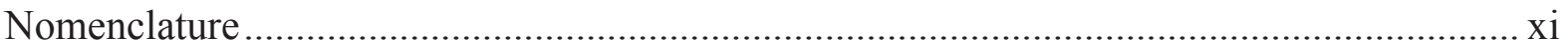

Chapter $1 \quad$ Introduction and Background ................................................................... 1

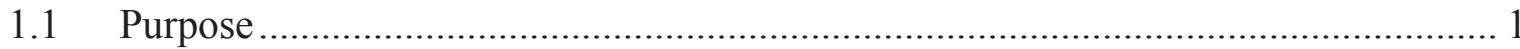

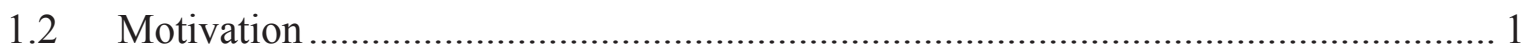

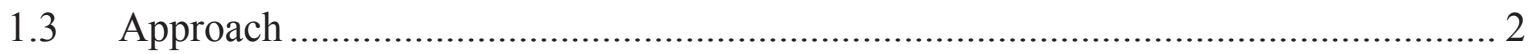

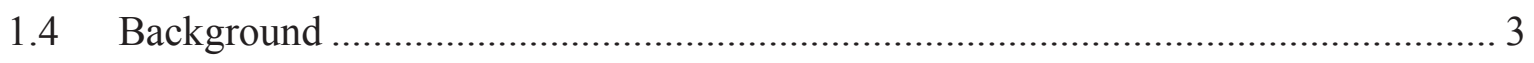

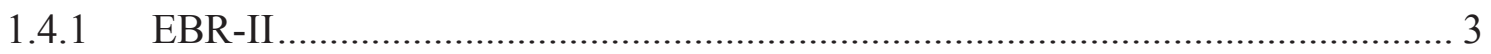

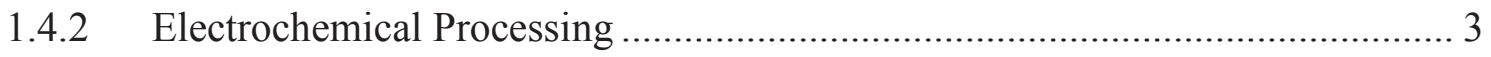

1.4.3 Electrolytic Oxide Reduction.................................................................... 4

1.4.4 Cold Finger Crystal Growth Separation ...................................................... 6

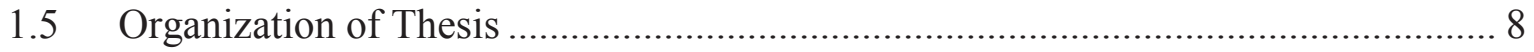

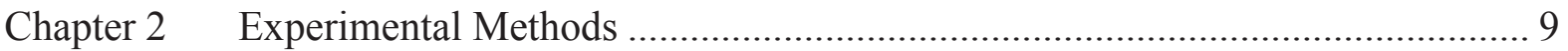

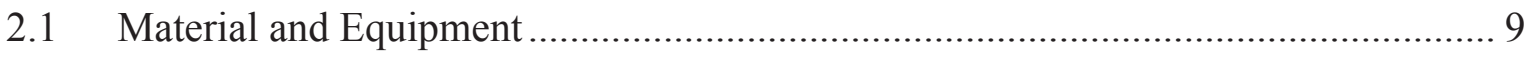

2.1.1 Cold Finger Design ............................................................................ 9

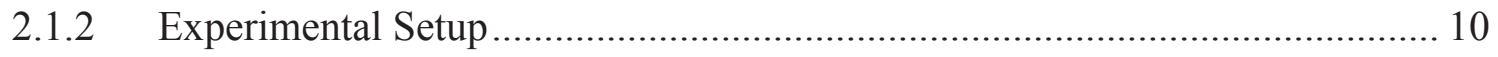

$2.2 \quad$ Experimental Procedures............................................................................... 13

2.2.1 Cold Finger Melt Crystallization Separation ............................................. 13 
2.2.2 Crystal Characterization and Preparation ................................................ 16

2.2.3 Sample Analysis and Preparation .......................................................... 17

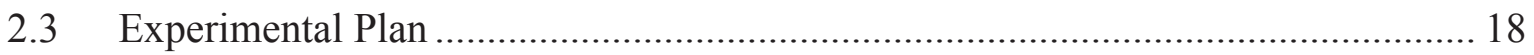

Chapter $3 \quad$ Experimental Results and Discussion ................................................ 20

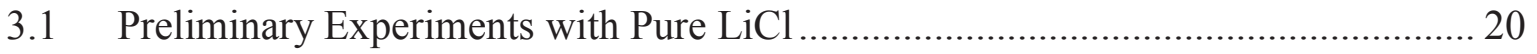

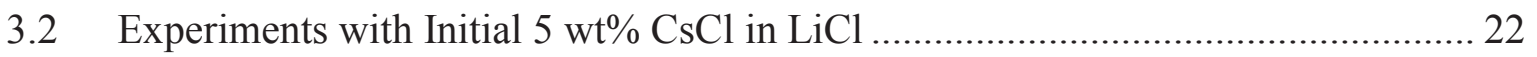

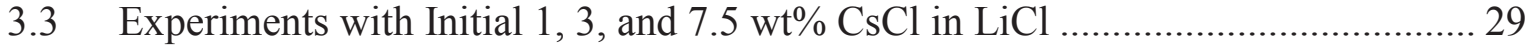

3.4 Potential Scale-up and Application ............................................................... 32

3.5 Crystal Growth Rate Correlations .................................................................. 34

Chapter 4 Conclusions and Recommendations...................................................... 50

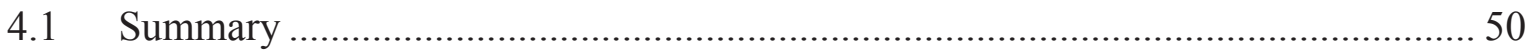

4.2 Recommendations for Future Work ......................................................... 51

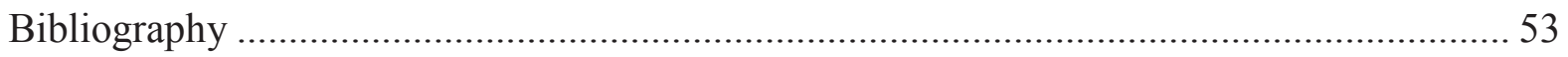

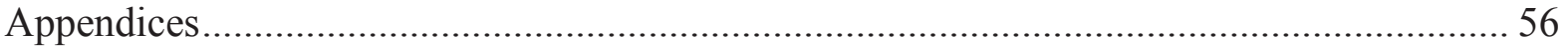

Appendix A - Experimental Mass Data ............................................................. 56

Appendix B - Experimental ICP-MS Concentration Data ....................................... 58

Appendix C - Calculated Parameters of Dimensionless Groups.................................... 61

Appendix D - Experimental Dimensionless Correlation Parameters ............................... 63

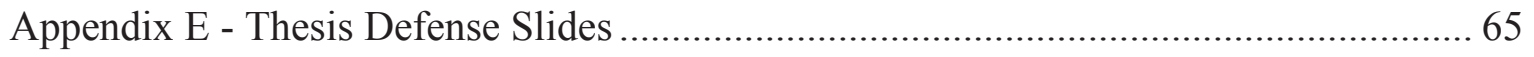




\section{List of Figures}

Figure 1.1: Conceptual schematic of current pyroprocessing technology. .......................... 4

Figure 1.2: Conceptual schematic of proposed advanced pyroprocessing technology with

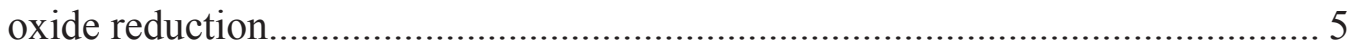

Figure 1.3: Diagram of the oxide reduction process of reducing oxide fuel to metal form... 6

Figure 1.4: Fact-Sage LiCl-CsCl binary phase diagram. ................................................ 8

Figure 2.1: Photograph of manufactured cold finger. ................................................. 9

Figure 2.2: Assembly and detailed part drawings of the cold finger design. .................... 10

Figure 2.3: Experimental setup in MBraun glovebox located in the Radiochemistry

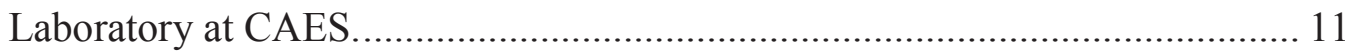

Figure 2.4: Cutaway diagram of experimental setup................................................. 12

Figure 2.5: Cold finger alignment diagram. .......................................................... 15

Figure 2.6: Experimental crystal representation showing color regions and dimensions

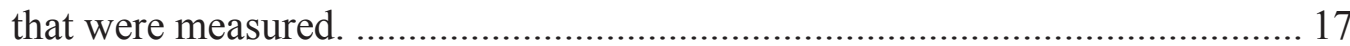

Figure 3.1: Crystal mass vs. crystal growth time for pure $\mathrm{LiCl}$ experiments at an

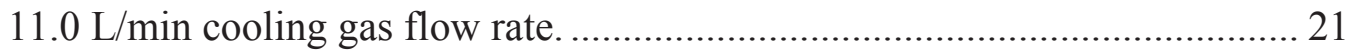

Figure 3.2: A montage of pure $\mathrm{LiCl}$ experiments for varying growth times at an $11.0 \mathrm{~L} / \mathrm{min}$ cooling gas flow rate. 21

Figure 3.3: Crystal mass vs. cooling gas flow rate for pure $\mathrm{LiCl}$ experiments at a $30 \mathrm{~min}$ crystal growth time.

Figure 3.4: A montage of pure $\mathrm{LiCl}$ experiments for varying cooling gas flow rates at a 30 min crystal growth time.

Figure 3.5: Crystal concentration results for experiments performed at an initial bulk concentration of $5 \mathrm{wt} \% \mathrm{CsCl}$ in $\mathrm{LiCl}$..

Figure 3.6: Crystal mass comparison for pure $\mathrm{LiCl}(\mathrm{A}$ and $\mathrm{B}$ ) and $\mathrm{LiCl}$ with an initial $5 \mathrm{wt} \% \mathrm{CsCl}(\mathrm{C}$ and $\mathrm{D})$ at the same cold finger operating conditions and parameters with lines highlighting an opaque residue drip formation. 25

Figure 3.7: Mass vs. time for crystal and drip with an initial $5 \mathrm{wt} \% \mathrm{CsCl}$ in $\mathrm{LiCl}$ for varying cooling gas flow rates compared to pure $\mathrm{LiCl}$ experiments at a cooling gas flow rate of $11.0 \mathrm{~L} / \mathrm{min}$ 
Figure 3.8: Purified crystal and drip concentration vs. crystal growth time for initial $5 \mathrm{wt} \% \mathrm{CsCl}$ in $\mathrm{LiCl}$ for varying cooling gas flow rates.

Figure 3.9: Purified crystal and drip concentration vs. crystal growth rate for initial $5 \mathrm{wt} \% \mathrm{CsCl}$ in $\mathrm{LiCl}$ and 15 min crystal growth times for varying cooling gas flow rates

Figure 3.10: Drip concentration vs. bulk concentration for experiments performed at initial concentrations of 1,3 , and $7.5 \mathrm{wt} \% \mathrm{CsCl}$ in $\mathrm{LiCl}$ with 15 min crystal growth times for varying cooling gas flow rates.

Figure 3.11: Purified crystal and drip mass vs. initial bulk concentration for experiments performed with 15 min crystal growth times for varying cooling gas flow rates.

Figure 3.12: Purified crystal and drip concentration vs. initial bulk concentration for experiments performed with 15 min crystal growth times for varying cooling gas flow rates.

Figure 3.13: Scale-up scenario and application of cold finger melt crystallization with existing bench scale oxide reduction equipment at INL [5].

Figure 3.14: Dimensionless crystal mass vs. respective dimensionless parameter including the effects of heat, mass, and heat \& mass transfers for experimental data and correlation predictions of experiments with varying initial compositions, crystal growth times, and cooling gas flow rates.

Figure 3.15: Experimental vs. correlation dimensionless crystal mass, where the correlation is a function of dimensionless $G r, P r$, and $S c$ for experiments with varying initial compositions, crystal growth times, and cooling gas flow rates.

Figure 3.16: Residual vs. predicted $\eta$ for: (A) heat transfer correlation, (B) mass transfer correlation, and $(\mathrm{C})$ heat and mass transfer correlation.

Figure 3.17: $\eta$ vs. $G r$ for new: (A) heat transfer, (B) mass transfer, and (C) heat and mass transfer correlations fitted to experimental data performed with initial 1, 3, and $7.5 \mathrm{wt} \% \mathrm{CsCl}$.

Figure 3.18: $\eta$ vs. $G r$ for new correlations predicting experimental data performed with initial $5 \mathrm{wt} \% \mathrm{CsCl}$. 


\section{List of Tables}

Table 2.1: Experiments performed for this work....................................................... 19

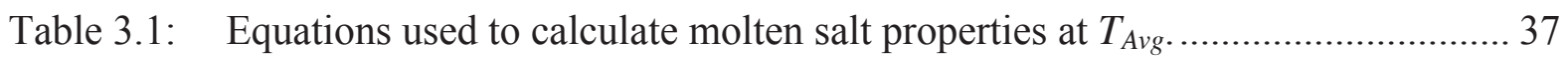

Table 3.2: $\quad$ Three proposed dimensionless purified crystal mass correlations with respective $a, b$, correlation coefficient $(R)$, correlation of determination $\left(R^{2}\right)$, and probability function $\left(P_{C}\right)$ values discussed later. .................................. 39

Table 3.3: The correlation coefficients $(R)$, coefficients of determination $\left(R^{2}\right)$, and probability functions $P_{C}(R, N)$ for all three correlations.............................. 42

Table 3.4: Average $h_{t h}$ and $h_{m}$ for the three correlations............................................. 44

Table 3.5: Three new proposed dimensionless purified crystal mass correlations with respective $a, b, \mathrm{c}, \mathrm{d}, R^{2}$, and $R M S D$ values for experiments performed with initial 1,3 , and $7.5 \mathrm{wt} \% \mathrm{CsCl}$.

Table 3.6: $\quad R^{2}$ values for new correlations fitted to experiments performed with initial 1,3 , and $7.5 \mathrm{wt} \% \mathrm{CsCl}$ for various combinations progressively including more cooling gas flow rate data sets.

Table 3.7: Three new proposed dimensionless purified crystal mass correlations with respective $a, b, \mathrm{c}, \mathrm{d}, R^{2}$, and $R M S D$ values of predicting experiments performed with initial $5 \mathrm{wt} \% \mathrm{CsCl}$. 


\section{Nomenclature}

\section{Chemical Symbols}

$\begin{array}{ll}\mathrm{Ar} & \text { Argon } \\ \mathrm{Cs} & \text { Cesium } \\ \mathrm{CsCl} & \text { Cesium chloride } \\ \mathrm{e}^{-} & \text {Electron } \\ \mathrm{HNO}_{3} & \text { Nitric acid } \\ \mathrm{H}_{2} \mathrm{O} & \text { Water } \\ \mathrm{Li}^{\mathrm{LiCl}} & \text { Lithium } \\ \mathrm{Li}_{2} \mathrm{O} & \text { Lithium chloride } \\ \mathrm{MgO} & \text { Lithium oxide } \\ \mathrm{O} & \text { Magnesium oxide } \\ \mathrm{O}_{2} & \text { Oxygen } \\ \mathrm{Pt} & \text { Oxygen gas } \\ \mathrm{Sr} & \text { Platinum } \\ \mathrm{U} & \text { Strontium } \\ \mathrm{UO} & \text { Uranium } \\ & \text { Uranium dioxide }\end{array}$

$\begin{array}{lll}\text { Symbol Description Unit } & \text { Un }\end{array}$

a Constant in correlations none

$b \quad$ Constant in correlations none

$B i$ Dimensionless Biot number none

c Constant in correlations none

$c_{P} \quad$ Specific heat capacity of molten salt $\quad \mathrm{J} /(\mathrm{kg} \mathrm{K})$

$\begin{array}{lll}c_{P c} & \text { Specific heat capacity of solid crystal } & \mathrm{J} /(\mathrm{kg} \mathrm{K})\end{array}$

d Constant in correlations none

$D_{A B} \quad$ Species diffusion coefficient $\quad \mathrm{m}^{2} / \mathrm{s}$

Fo Dimensionless Fourier number none 
$g$

Gr

$h_{c f}$

$h_{m}$

$h_{t h}$

$\mathrm{Ja}$

$k$

$k_{c}$

$L$

Le

$m$

$m_{0}$

$n$

$N$

$\mathrm{Nu}$

$P_{C}$

$\operatorname{Pr}$

$R$

$R^{2}$

$R a$

RMSD

Sc

Sh

$T_{A v g}$

$T_{\text {Bulk }}$

$T_{\text {Freeze }}$

$\alpha$

$\beta$

$\Gamma(k)$
Gravitational constant

Dimensionless Grashof number

Latent heat of fusion for $\mathrm{LiCl}$

Mass transfer coefficient

Heat transfer coefficient

Dimensionless Jakob number

Thermal conductivity of molten salt

Thermal conductivity of solid crystal

Characteristic length

Dimensionless Lewis number

Mass of purified crystal

Mass of initial $\mathrm{LiCl}$ in $30 \mathrm{~g}$ of $\mathrm{LiCl}-\mathrm{CsCl}$

Number of experimental data points

Number of uncorrelated experimental data points

Dimensionless Nusselt number

Probability functions

Dimensionless Prandtl number

Correlation coefficient

Coefficients of determination

Dimensionless Rayleigh number

Root mean square percent change deviation

Dimensionless Schmidt number

Dimensionless Sherwood number

Average temperature of molten salt between bulk and

solid/liquid interface

Bulk temperature of molten salt

Freezing temperature of molten salt

Thermal diffusivity of molten salt

Thermal expansion coefficient

The Euler gamma function to the factorial function $k$ ! $\mathrm{m} / \mathrm{s}^{2}$

none

$\mathrm{m} / \mathrm{s}$

$\mathrm{m} / \mathrm{s}$

$\mathrm{W} /\left(\mathrm{m}^{2} \mathrm{~K}\right)$

none

$\mathrm{W} /(\mathrm{m} \mathrm{K})$

$\mathrm{W} /(\mathrm{m} \mathrm{K})$

m

none

$\mathrm{kg}$

$\mathrm{kg}$

none

none

none

none

none

none

none

none

none

none

none

K

K

K

$\mathrm{m}^{2} / \mathrm{s}$

$\mathrm{K}^{-1}$

none 


\section{Chapter 1 Introduction and Background}

\subsection{Purpose}

The purpose of this thesis research is to elucidate a fundamental study of cold finger separation to optimize the separation of cesium chloride $(\mathrm{CsCl})$ from a lithium chloride $(\mathrm{LiCl})$ electrolyte. Experimental data sets showing effects of initial $\mathrm{CsCl}$ concentration, cold finger coolant flow rate, and separation time will aid in future optimization of process scaleup.

\subsection{Motivation}

The management and disposal of used nuclear fuel is a growing challenge in the United States (U.S.) today due to its continued use of a once through fuel cycle. In 1977, a once through fuel cycle was adopted because of President Carter's veto preventing the authorization to construct a breeder reactor and reprocessing facility in Barnwell, South Carolina, for non-proliferation reasons [1]. The once through fuel cycle is wasteful as supported by the fact that less than five percent of used uranium produces electric power [2]. In 2001, it was recommended by President Bush's National Energy Policy that the U.S. consider developing cleaner and more proliferation-resistant reprocessing technologies [1]. In response to President Bush's National Energy Policy recommendation, the U.S. Department of Energy (DOE) has since organized The Office of Nuclear Energy's Fuel Cycle Research and Development (FCR\&D) program in an effort to close the fuel cycle by recycling used nuclear fuel [3].

Pyroprocessing is one reprocessing technology that the U.S. DOE is exploring because of its non-proliferation and safety benefits. The pyroprocessing of both oxide and metallic uranium fuel has non-proliferation benefits because it is more difficult to separate out pure plutonium metal than it is for aqueous processing. In addition, it has some safety benefits over aqueous reprocessing such as more stable process liquids and improved criticality safety [4].

Oxide reduction is the head end process of pyroprocessing that enables the process to accommodate reprocessing of oxide fuel $\left(\mathrm{UO}_{2}\right)$. Here, electrolytic oxide reduction is an 
electrochemical process involving a cathode, anode, and electrolyte that reduces oxide fuel to metallic form. The electrolyte is a lithium chloride - lithium oxide $\left(\mathrm{LiCl}-\mathrm{Li}_{2} \mathrm{O}\right)$ salt which becomes contaminated over time with fission products that diffuse from the used nuclear fuel into the electrolyte during operation [5].

Cold finger crystal growth separation is a proposed method for removing fission products from oxide reduction electrolyte in an effort to reduce nuclear waste volume. Cold finger crystal growth separation is similar to a melt crystallization process called layer crystallization that concentrates used electrolyte as purified electrolyte is solidified on a cooled wall and removed. Layer crystallization was proposed and explored by the Korea Atomic Energy Research Institute (KAERI) and was experimentally proven to minimize the amount of lithium chloride $(\mathrm{LiCl})$ salt waste [6]. Despite the successful demonstration of this technique, there are still missing fundamental aspects to be explored such as heat and mass transfers and process optimization. These fundamental aspects provide the motivation for studying cold finger melt crystallization separation to optimize the process and safely reduce oxide reduction electrolyte waste.

\subsection{Approach}

The experimental approach is to investigate a $\mathrm{LiCl}$ salt contaminated with cesium chloride $(\mathrm{CsCl})$. $\mathrm{LiCl}$ was selected for the oxide reduction electrolyte to simplify the system because only $1 w t \%$ (U.S.) [5] and 3wt\% (South Korea) [7] of the $\mathrm{LiCl}-\mathrm{Li}_{2} \mathrm{O}$ electrolyte is lithium oxide $\left(\mathrm{Li}_{2} \mathrm{O}\right)$. To further simplify the system non-radioactive $\mathrm{CsCl}$ was selected to represent Group I actinides because of its abundant accumulation in the oxide reduction electrolyte [5]. Experiments were performed at varying initial bulk $\mathrm{CsCl}$ concentration, cold finger cooling gas flow rate, and crystal growth separation time. For each experiment the cooling gas exhaust and molten salt bulk temperatures were measured over time. The cold finger cooling gas was manually controlled via a needle valve flow control. The cooling gas was metered manually instead of electronically in an effort to simplify the experimental setup in the glovebox and eliminate unnecessary electric feed-through into the glovebox. The chloride salts used in this study are hygroscopic, so experiments and sample preparation were performed in an inert argon (Ar) atmosphere glove box. To be compatible with the glove box atmosphere, argon was used as the cold finger cooling gas. Concentration data was 
obtained via Inductively Coupled Plasma Mass Spectroscopy (ICP-MS) for crystal formations formed on cold finger and $\mathrm{CsCl}$ concentrated salt left behind in crucible.

Three crystal growth rate correlations were developed to better understand crystal growth rate behavior and determine the rate controlling physics of the cold finger melt crystallization separation process. Three scenarios (heat, mass, and both heat \& mass transfers) were proposed with the use of dimensionless Grashof, Prandtl, and Schmidt numbers where the length scale is the cubed root of purified crystal volume. The correlation took the form of a conventional Nusselt or Sherwood correlation for a natural convective system.

\subsection{Background}

\subsubsection{EBR-II}

The Experimental Breeder Reactor-II (EBR-II) was designed and constructed to advance the technology of Experimental Breeder Reactor-I (EBR-I) with an objective of future commercialization [8]. EBR-II is a sodium cooled fast reactor located at the Idaho National Laboratory (INL), formerly known as Argonne National Laboratory-West (ANLWest) [9]. The reactor was in power operation from 1964 to 1994 and had a maximum power level of 62.5 MWt while generating $20 \mathrm{MWe}$ [8] and [9]. The reactor was designed to operate on recycled fuel that was reprocessed onsite to close this reactors fuel cycle. From 1965 to 1969 , used EBR-II fuel was reprocessed with a melt refining operation at the ANLWest Fuel Cycle Facility (FCF) and from 1969 to 1994 was treated at the Idaho Chemical Processing Plant. In 1994, the fuel began being reprocessed with a pyrochemical process at the ANL-West FCF, currently known as INL FCF [9].

\subsubsection{Electrochemical Processing}

Pyroprocessing is a "dry" high temperature electrochemical process of treating used nuclear fuel (see Figure 1.1). The term "dry" refers to a process that does not involve any aqueous components. Pyroprocessing in the U.S. was originally developed to treat used metallic fuel produced by EBR-II. At the heart of the pyroprocess is the electrorefiner where metallic fuel is oxidized in an anode basket, dissolved into a molten salt electrolyte, and reduced at a cathode where uranium is deposited and recovered for reuse. The fuel assembly 
cladding, noble metals, and fission products remaining in the anode basket are blended with zirconium and cast into a metal waste form. The used electrolyte contaminated with fission products, rare earths, and transuranics (TRU) is blended with zeolite and glass, then cast into a ceramic waste form [ [10], [11], [12], \& [13]].

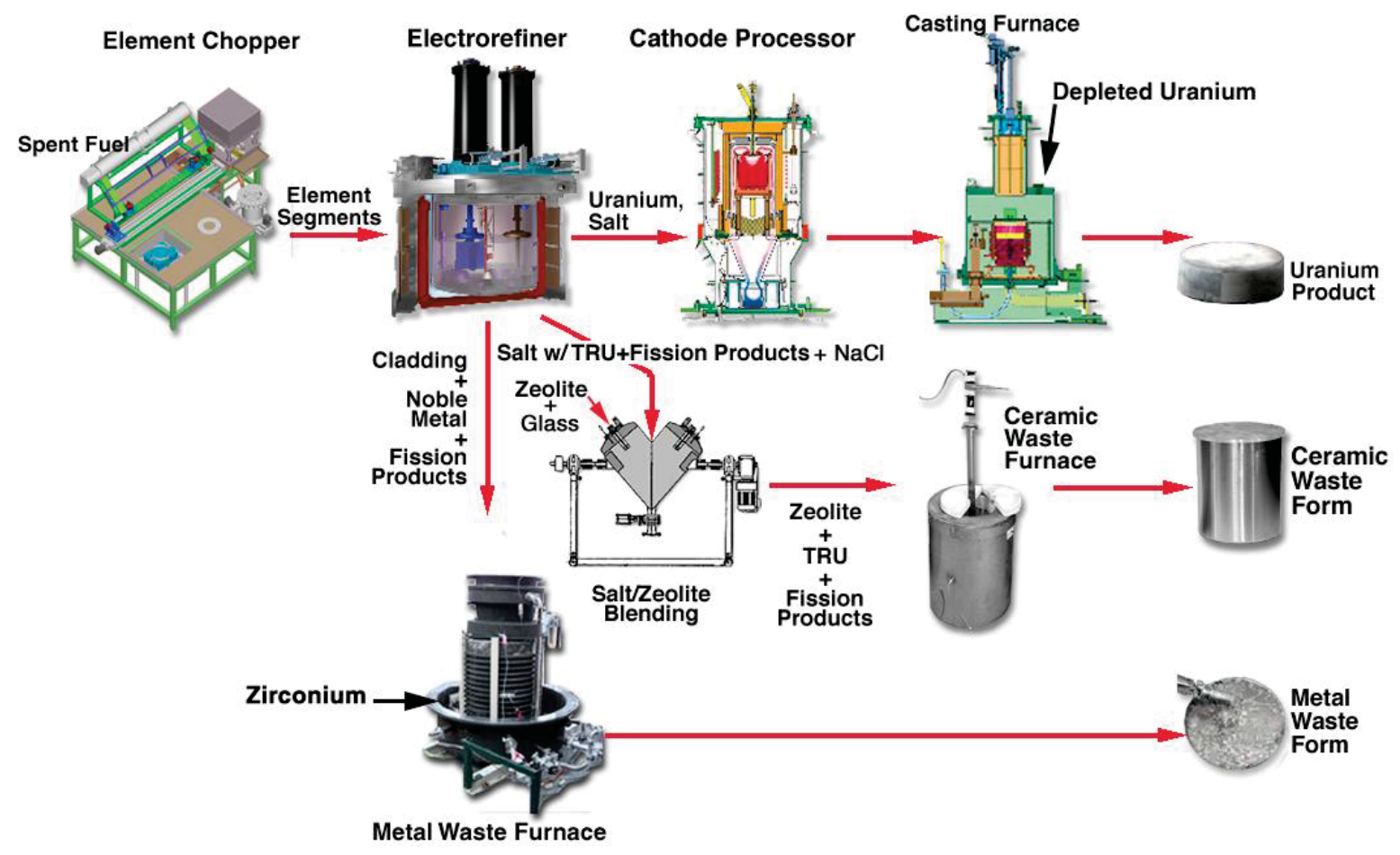

Figure 1.1: Conceptual schematic of current pyroprocessing technology.

\subsubsection{Electrolytic Oxide Reduction}

Although pyroprocessing in the U.S. was originally developed to treat metallic fuel, it has been adapted to treat commercial oxide fuel with a head-end step of oxide reduction just before electrorefining (see Figure 1.2). Oxide fuel is used in all U.S. commercial reactors, motivating the adaptation of pyroprocessing to treat oxide fuel. Electrolytic Oxide Reduction is a process that reduces oxide fuel to its metal form through electrochemistry. In the U.S., the reduction takes place in a molten salt electrolyte $\left(\mathrm{LiCl}-\mathrm{Li}_{2} \mathrm{O} 1 \mathrm{wt} \% \mathrm{Li}_{2} \mathrm{O}\right)$ at $650^{\circ} \mathrm{C}$, where a submerged stainless steel cathode basket filled with crushed oxide fuel is reduced to metal at the cathode while oxygen gas is formed at the surface of a submerged platinum $(\mathrm{Pt})$ wire 
anode (see Figure 1.3). The reactions that take place at the cathode and anode, to produce the aforementioned net reaction, are as follows.

Cathode: $\quad \mathrm{UO}_{2}+4 \mathrm{e}^{-} \rightarrow \mathrm{U}+2 \mathrm{O}^{2-}$

Anode: $\quad 2 \mathrm{O}^{2-} \rightarrow \mathrm{O}_{2}(\mathrm{~g})+4 \mathrm{e}^{-}$

Net Reaction: $\mathrm{UO}_{2} \rightarrow \mathrm{U}+\mathrm{O}_{2}(\mathrm{~g})$

Experiments have shown that, as oxide fuel is reduced to metal in the oxide reduction process, group I fission products such as cesium partition from the fuel and diffuse into the molten salt electrolyte [5]. Fission product accumulation in the molten salt electrolyte affects the oxide reduction process efficiency by changing the melting temperature of the electrolyte. It is reasonable to say based on phase equilibrium that as fission products build-up in the molten salt electrolyte its melting temperature decreases. To prevent faster cooling rates required by lower melting temperatures, it is necessary to replace and dispose of contaminated electrolyte.

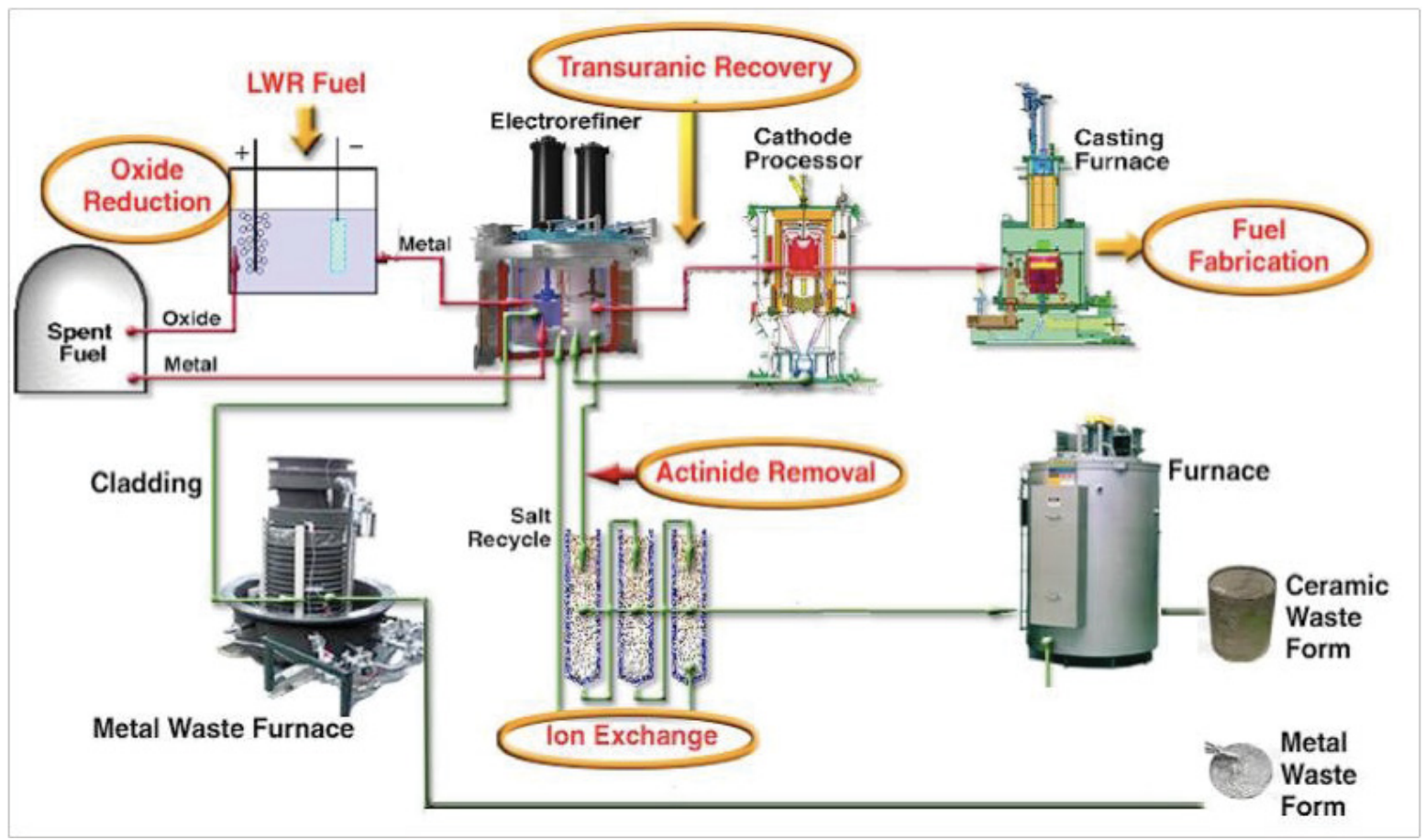

Figure 1.2: Conceptual schematic of proposed advanced pyroprocessing technology with oxide reduction. 


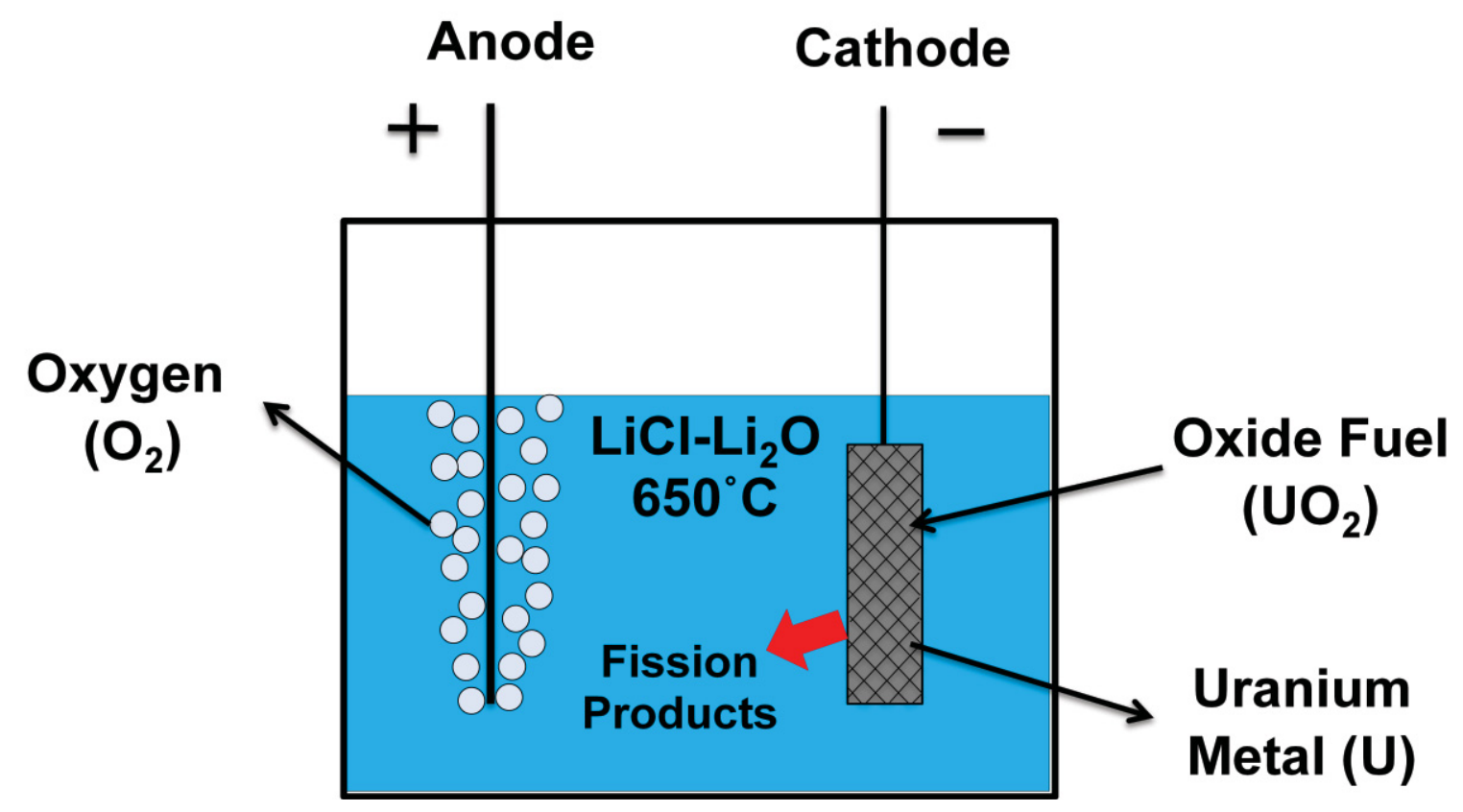

Figure 1.3: Diagram of the oxide reduction process of reducing oxide fuel to metal form.

\subsubsection{Cold Finger Crystal Growth Separation}

Two melt crystallization separating techniques have been explored to reduce used electrolyte waste volume and lower operating expenses by recycling used electrolyte. Zone freezing has been explored by KAERI and University of Idaho (U of I) where KAERI studied a LiCl salt system [6] and U of I studied a LiCl-KCl salt system [14]. Layer crystallization, a separation technique similar to cold finger crystal growth, has been explored by KAERI for a $\mathrm{LiCl}$ salt system [6].

Cold finger and layer crystallization have two major benefits over zone freezing. The first benefit is they are faster while maintaining comparable separation purity and the second is they are easier to scale-up for commercial applications. The zone freezing process requires upward translation of a batch of used electrolyte from a hot zone to a cold zone to slowly freeze purified electrolyte from top to bottom. The solid crystalline formation is purer at the top than at the bottom because the electrolyte thermodynamically solidifies before the fission product contaminate in the electrolyte. As the electrolyte freezes from top to bottom the contaminate is forced away from the solid phase into the melt phase leaving the crystalline formation more concentrated in contaminate nearer the bottom of the solid crystalline 
formation. This process is reported to be long with translation velocities from a hot to a cool zone ranging from 1.7 to $5.7 \mathrm{~mm} / \mathrm{h}$ for KAERI [6] and 1.8 to $5.0 \mathrm{~mm} / \mathrm{h}$ for $\mathrm{U}$ of I [14] resulting in process times ranging from 1 to 3 days compared to layer crystallization process times reported to be less than $1 \mathrm{~h}$ by KAERI [6]. Zone freezing is difficult to scale-up because increasing batch size increases the time required for heat to diffuse away from the center of the batch requiring slower translation velocities to maintain comparable separation purities to lab scale results. On the contrary, cold finger and layer crystallization can be scaled up by simply increasing the effective cooling surface area.

The current study, proposed by $\mathrm{U}$ of $\mathrm{I}$, is a cold finger melt crystallization separation technique that concentrates $\mathrm{CsCl}$ in a $\mathrm{LiCl}-\mathrm{CsCl}$ salt system as solid $\mathrm{LiCl}$ is removed via a cold finger. The cold finger is cooled to the melting temperature of $\mathrm{LiCl}\left(605^{\circ} \mathrm{C}\right)$ to selectively freeze $\mathrm{LiCl}$ to the cold finger so it can be removed as purified electrolyte [15]. Melt crystallization techniques are based on principles of thermodynamic phase change and phase equilibrium. The separation theory is best described with the use of a binary phase diagram of $\mathrm{LiCl}-\mathrm{CsCl}$ provided by Fact-Sage [16] (see Figure 1.4). The binary phase diagram includes additional figures that show how molten salt electrolyte is concentrated with $\mathrm{CsCl}$ as $\mathrm{LiCl}$ is selectively removed, via the cold finger, over time. An initial time and concentration of $\mathrm{CsCl}$ is depicted as stage one. Stage two represents an intermediate time where the molten salt is more concentrated having a lower liquidus temperature. Stage three would be the final time where a relatively pure $\mathrm{LiCl}$ crystal is removed from the $\mathrm{CsCl}$ concentrated melt for reuse. The cold finger was designed to have a controlled surface temperature that targets the liquidus melting temperature of a given molten salt composition in order to selectively freeze out $\mathrm{LiCl}$ from contaminated oxide reduction electrolyte. KAERI has shown for their similar process of layer crystallization that $90 \%$ of processed electrolyte can be recycled back to the electrolytic oxide reduction process at crystal growth rates less than $5 \mathrm{~g} / \mathrm{min}$ with at least $90 \% \mathrm{LiCl}$ electrolyte purity [6]. 


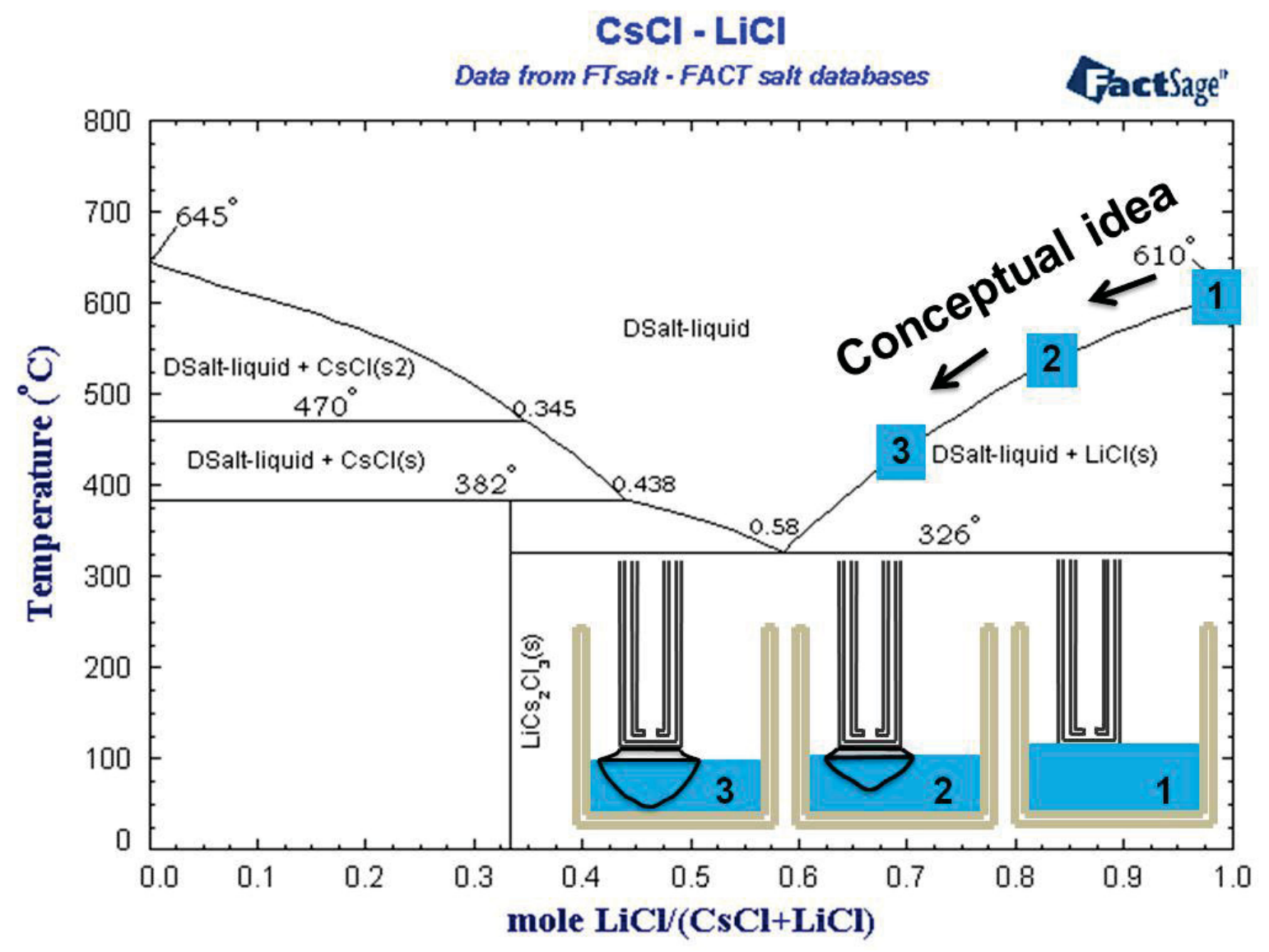

Figure 1.4: Fact-Sage LiCl-CsCl binary phase diagram.

\subsection{Organization of Thesis}

Chapter 1 of this thesis provides the purpose, motivation, and background information of this work. Chapter 2 describes experimental conditions, cold finger design, experimental setup, equipment, procedures, and experiments performed. In Chapter 3, the experimental results are presented, analyzed, discussed, and compared to cross-validate the proposed correlations. Chapter 4 contains a summary and recommendations for this thesis work. 


\section{Chapter 2 Experimental Methods}

\subsection{Material and Equipment}

\subsubsection{Cold Finger Design}

The cold finger was designed and manufactured as an instrument to selectively solidify LiCl by controlling the cold finger surface temperature to target LiCl's freezing temperature. The cold finger design consists of two coaxial stainless steel tubes positioned with six set screws (see Figure 2.1). The smaller diameter tube is held in position within the large diameter tube by the set screws. The large diameter tube is closed at the bottom with a welded disk and the smaller diameter tube is caped at its bottom with a welded disk equipped with a $1 / 8$ " hole drilled in its center. The caped ends of the tubes were positioned 1/32" from each other allowing for a coolant passage. The coolant flow through the cold finger enters the cold finger from the top of the smaller diameter tube and exits the annular opening between the tubes at the top of the large diameter tube (see Figure 2.2). This cold finger was designed for simplicity, ease of use, and ease of cleaning; as it is to eventually be used in a radiological environment. Before the cold finger was placed into operation it was wiped clean of any oil from fabrication with acetone and then heated for 1 hour in a furnace at $650^{\circ} \mathrm{C}$ to further clean and dry it.

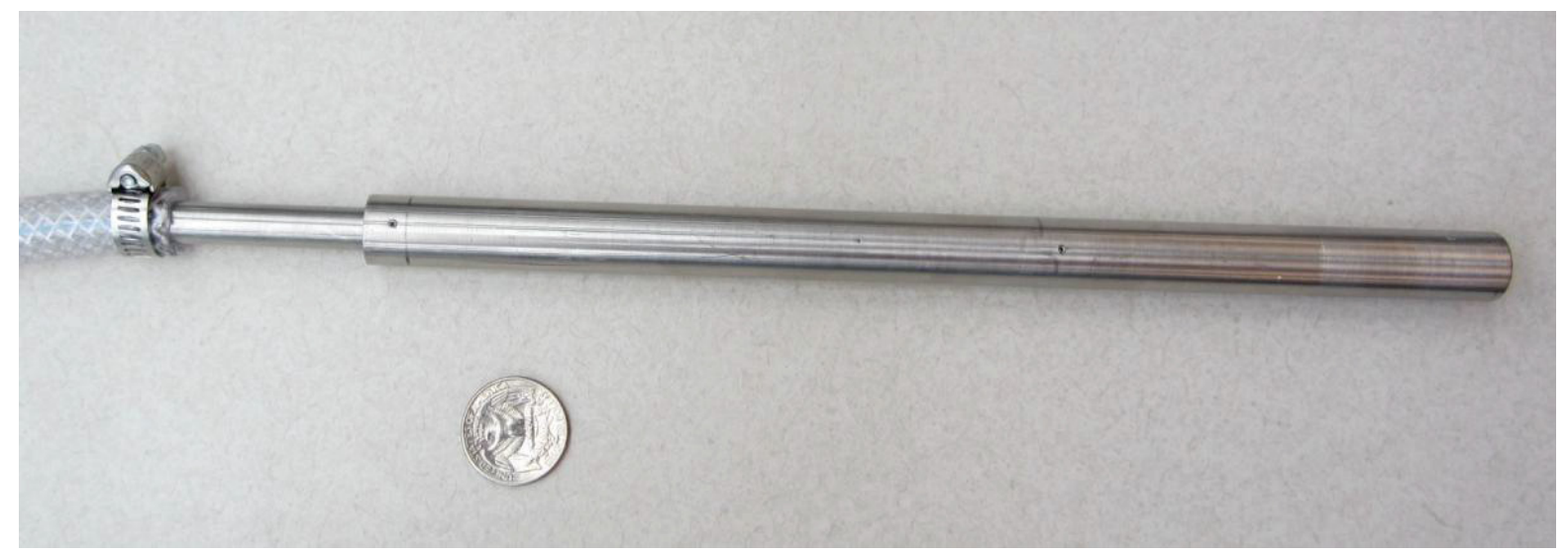

Figure 2.1: Photograph of manufactured cold finger. 


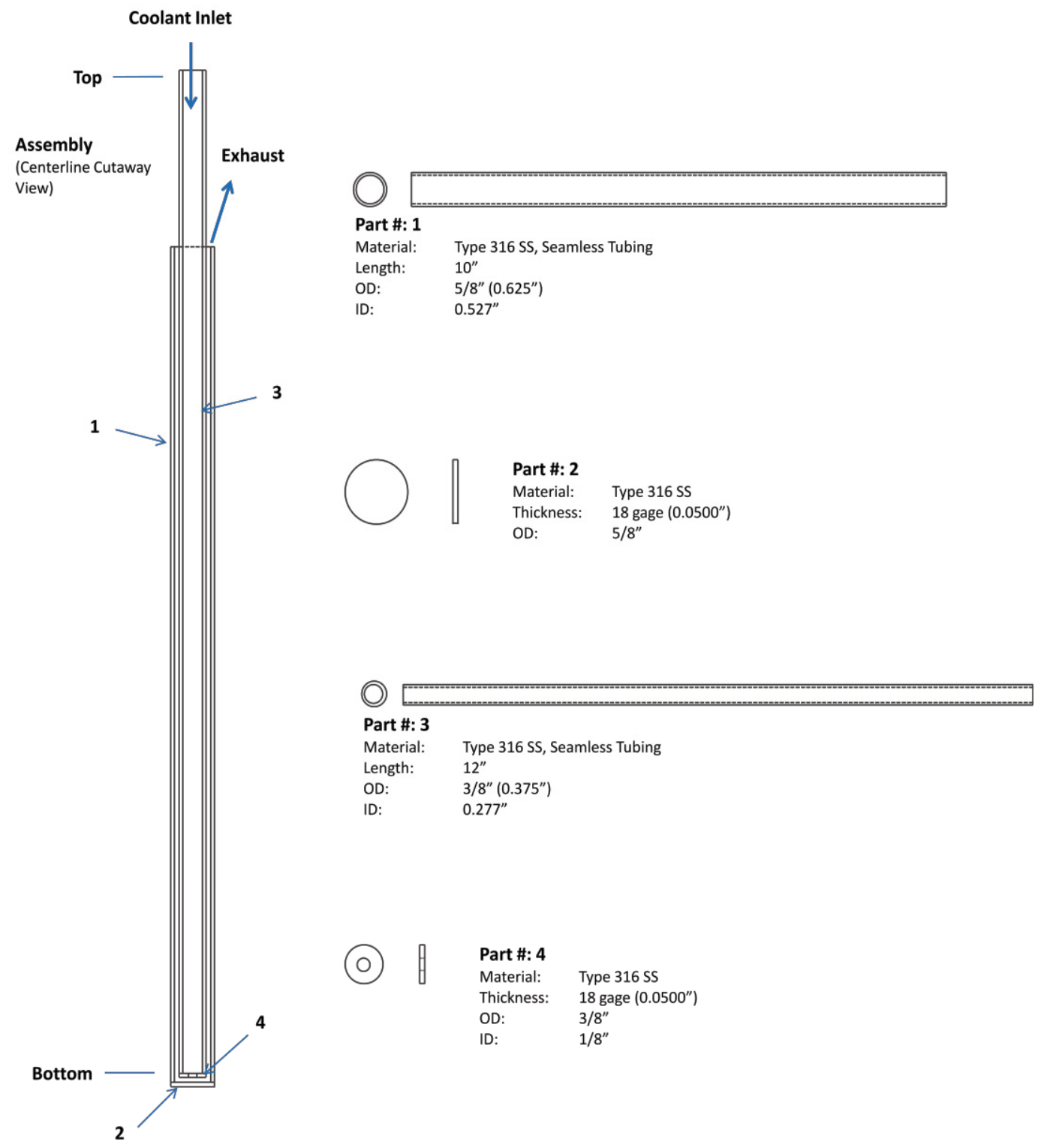

Figure 2.2: Assembly and detailed part drawings of the cold finger design.

\subsubsection{Experimental Setup}

The experimental equipment was set up in an MBraun glovebox located in the Radiochemistry Laboratory at the Center for Advanced Energy Studies (CAES). The glovebox provides an inert argon gas atmosphere maintaining oxygen and moisture levels below $5 \mathrm{ppm}$. The experimental set up consists of an Air Gas flow meter, custom cold finger, Idaho Laboratory type-K thermocouples, Ozark Technical Ceramics magnesium 
oxide $(\mathrm{MgO})$ crucible, Alfa Aesar lithium chloride salt ( $\mathrm{LiCl})$, Aldrich Chemistry cesium chloride salt $(\mathrm{CsCl})$, Kerr Electro-Melt furnace, and type-K Omega thermometers (see Figure 2.3). The flow meter provides the desired amount of argon cooling gas required to cool the tip of the cold finger to the freezing temperature of $\mathrm{LiCl}$. The cold finger is placed in the crucible containing $30 \mathrm{~g}$ of molten salt so its tip is flush with the molten salt contained by the crucible measuring 2 inches in diameter and 2 inches in height (see Figure 2.4). Both Figure 2.3 and Figure 2.4 show three stainless steel discs mounted near the cooling gas exhaust opening of the cold finger. The three discs were added as heat shields and cooling fins to prevent melting of the tygon cooling gas supply hose. The experiments were operated and controlled at $650^{\circ} \mathrm{C}$, which is the same operating temperature for the oxide reduction process.
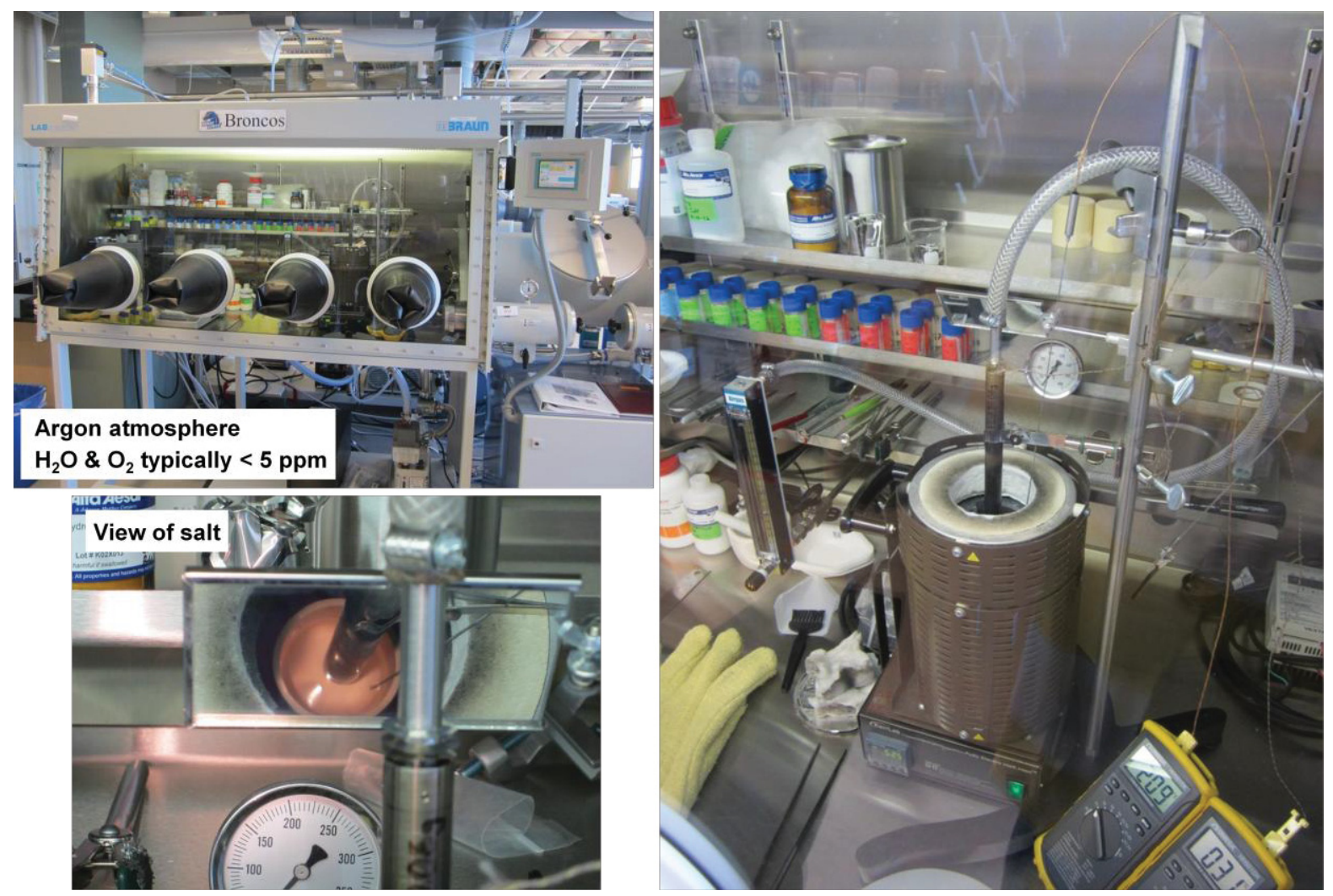

Figure 2.3: Experimental setup in MBraun glovebox located in the Radiochemistry Laboratory at CAES. 


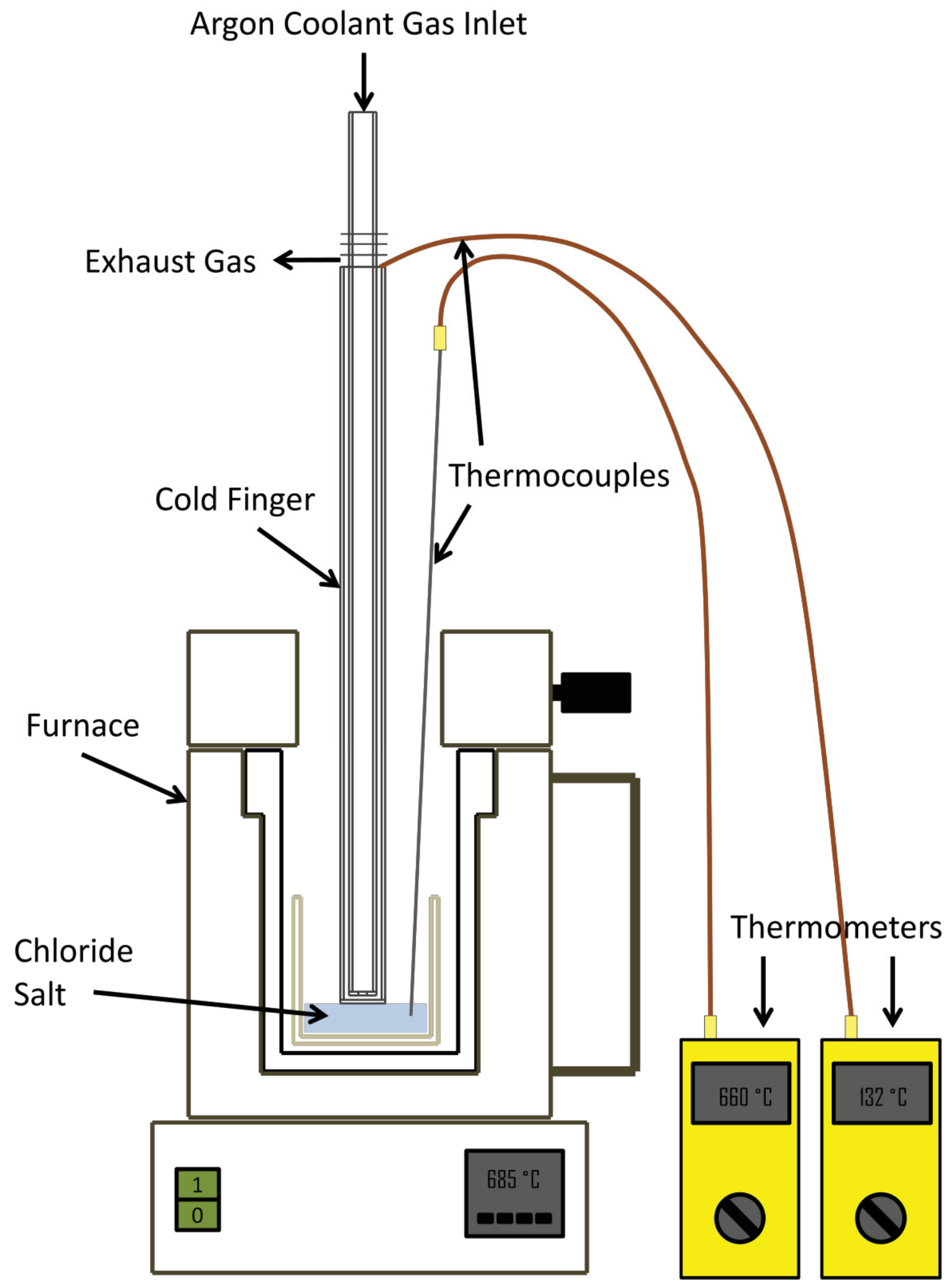

Figure 2.4: Cutaway diagram of experimental setup. 


\subsection{Experimental Procedures}

Routine procedures were followed for each experiment to ensure that experiments were consistent as to minimize variance between experiments. The importance for consistency between experiments is primarily due to the difficulty of sampling over time for one continuous experiment over time, creating a need to break one continuous experiment into multiple experiments for different time intervals within the overall experimental time interval. The multiple experiments provide time averages for the different time intervals within the overall time interval representing one continuous experiment over time. There were three major experimental procedures that were developed and followed. These are:

- The cold finger melt crystallization separation process

- The crystal characterization and preparation

- The sample preparation for ICP-MS elemental analysis

\subsubsection{Cold Finger Melt Crystallization Separation}

The experimental cold finger melt crystallization separation procedure is outlined and discussed as follows. In preparation for each experiment, the argon cooling gas supply was regulated to $100 \mathrm{psi}$ and the glove box pressure was brought to a gage pressure of zero units. The mass of the crucible was measured. Thirty grams of $\mathrm{LiCl}-\mathrm{CsCl}$ were weighed out with different concentrations of $\mathrm{CsCl}$ at $1,3,5$, and $7.5 \mathrm{wt} \%$. The measured mixture was then transferred into the crucible. The crucible containing the salt was placed in the melt furnace and a thermocouple was inserted into the salt. The cold finger's initial mass was measured, and then connected to the argon cooling gas supply hose with a stainless steel hose clamp. The cold finger was then mounted to a lab stand with a thermometer clamp so the cold finger was positioned above the furnace so as to not obstruct the furnace opening. The furnace opening was covered with two pieces of insulation to help the furnace heat up faster and reduce the amount of heat that goes into the glovebox environment. The maximum glovebox environment temperature is $40^{\circ} \mathrm{C}$ to ensure proper functionality of the glovebox. The furnace was powered on and set to reach a set point of $685^{\circ} \mathrm{C}$ resulting in a molten salt operating temperature of roughly $650^{\circ} \mathrm{C}$. A stop watch was started when the furnace was powered on to record various times to reach specific experimental conditions. The time for the furnace to 
reach its set point of $685^{\circ} \mathrm{C}$ (approximately $18 \mathrm{~min}$ ) and the time for the molten salt to reach $655^{\circ} \mathrm{C}$ (approximately $45 \mathrm{~min}$ ) was recorded to indicate any variance of equipment operation comparable to other experiments. Once the molten salt reached $655^{\circ} \mathrm{C}$ the insulation was removed and the molten salt mixture was manually stirred with a stainless steel stirring utensil for about 1 min to make sure that all salt was melted and well mixed. A small amount of salt mixture residue adhering to the stir rod was removed with the stir rod. The residue mass was considered to be insignificant with a mass estimated to be around $0.1 \mathrm{~g}$ and was not measured. After stirring, the insulation was placed back over the furnace opening and the molten salt was allowed to reach $655^{\circ} \mathrm{C}$ again. Once this temperature was reached, the cold finger was positioned so its bottom surface meets flush with the exposed surface of the molten salt (see Figure 2.4). The surface tension of the molten salt was approximated as pure $\operatorname{LiCl}\left(\gamma=125 \mathrm{mN} / \mathrm{m} @ 650^{\circ} \mathrm{C}\right)[17]$ and used in order to ensure consistency of cold finger positioning flush with the exposed surface of the molten salt. The surface of the molten salt was reflective, so with a mirror and flashlight the meniscus formed by the surface tension of the molten salt could be seen. By slightly moving the cold finger up and down, just after it made contact with the molten salt, the cold finger was visually positioned so the reflection of the meniscus appeared to have no curvature indicating that the cold finger and molten salt surface were flush with each other (see Figure 2.5). A second thermocouple was placed at the exhaust opening of the cold finger (see Figure 2.4). At this point the molten salt was allowed to heat for about 2 hours to further promote mixing and diffusion of species as a steady state condition was achieved. The mass of a glass sample vial with lid and sampling cup were measured to determine the mass of a bulk sample collected later. After the furnace had been on for a total of 3 hours, the bulk molten salt was assumed to be at its steady state operating temperature and ready for processing. 

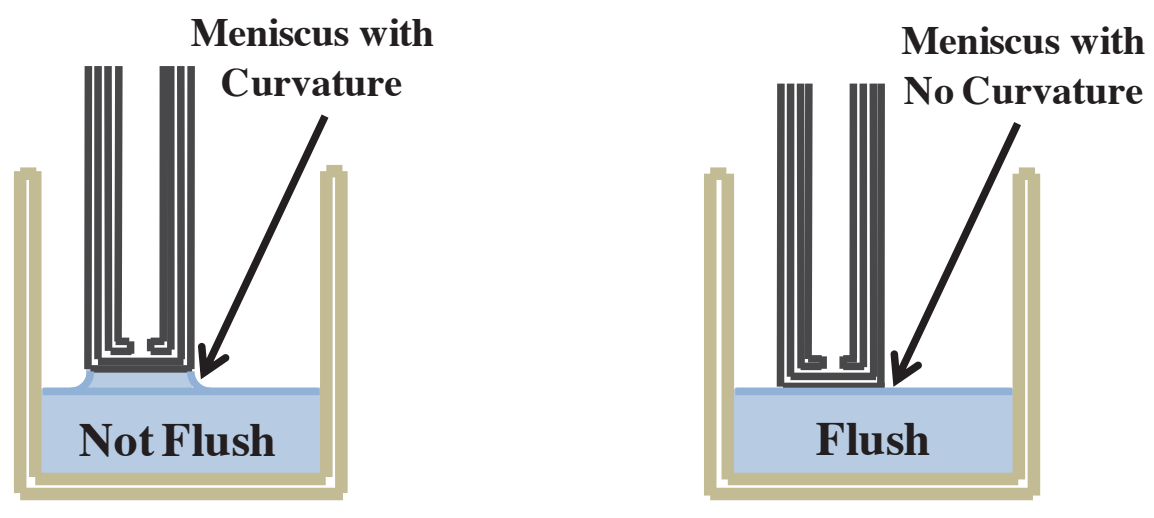

Figure 2.5: Cold finger alignment diagram.

With the completion of experimental preparation the cold finger melt crystallization process could begin. The steady state initial molten salt bulk and argon cooling gas exhaust temperatures (roughly $660^{\circ} \mathrm{C}$ and $131^{\circ} \mathrm{C}$ respectively) were recorded. Another stop watch was started as the argon cooling gas was turned on and regulated to different flow rates (7.4, 9.8, 12.3, and $14.9 \mathrm{~L} / \mathrm{min}$ ) by manually adjusting a needle valve flow controller.

Temperature measurements of the molten salt bulk and the argon cooling gas exhaust were recorded by hand at $1 \mathrm{~min}$ increments for different time intervals $(5,10,15$, and $30 \mathrm{~min})$. Midway through the crystal growth time, the glovebox atmosphere $\mathrm{H}_{2} \mathrm{O}$ and $\mathrm{O}_{2}$ levels (roughly $5 \mathrm{ppm}$ for both) were recorded along with its temperature (about $32^{\circ} \mathrm{C}$ ). At the end of the crystal growth time, the cold finger was removed from the molten salt by sliding it up in the lab stand clamp until the grown crystal was completely removed from the molten salt; however, its position is still inside the heat envelope of the furnace to reduce crystal cracking due to thermal shock. A bulk salt sample was collected with the previously weighed sample cup by dipping the sample cup into the molten salt. The bulk salt sample was allowed to cool then placed in the previously weighed vial to be weighed again to determine the bulk sample mass. The thermocouple previously placed in the molten salt was removed from the salt and left in the furnace well. This action was performed to (1) prevent it from freezing into the bulk salt as it cooled and (2) indicate the furnace well temperature during cool down. Once the thermocouple was removed from the molten salt the furnace was powered off and the furnace opening was covered with the two pieces of insulation. The furnace well was allowed to gradually cool to $575^{\circ} \mathrm{C}$ while cooling gas continued to cool the cold finger to ensure that the crystal did not melt away in the furnace. The cooling gas was shut off once 
the furnace well had cooled to $575^{\circ} \mathrm{C}$ which is about $30^{\circ} \mathrm{C}$ below the melting point of $\mathrm{LiCl}$ $\left(605^{\circ} \mathrm{C}\right)[15]$. The furnace, crystal, and salt were then cooled naturally to a safe and comfortable handling temperature of less than $50^{\circ} \mathrm{C}$, at which point the salt and crystal were removed from the furnace. The cool down process required at least 4 hours to cool to $50^{\circ} \mathrm{C}$ and usually cooled over night.

\subsubsection{Crystal Characterization and Preparation}

Nondestructive and destructive mass and dimension measurements were gathered. The masses of both the cold finger with crystal and the crucible with bulk salt were measured. These two mass measurements were used to determine the crystal mass growth rate and the remaining concentrated bulk salt mass. Dimensions a, b, d, and e shown in Figure 2.6 were measured to understand and locate the solid/liquid surface area interface throughout the crystal growth process. Dimension "c" was measured later once a portion of the crystal's edge was chipped away from the cold finger. Here, dimension "a" is the overall diameter of the crystal, "b" is the overall height of the crystal formation, "c" is the height the crystal grow up the side of the cold finger, " $\mathrm{d}$ " is the diameter of an opaque film of concentrated bulk salt that freezes at the bottom of the crystal in the form of a drip, and "e" is the distance between the crystals highest point up the side of the cold finger and its point of largest diameter. The white portion of the crystal drip was removed by a machining file (see Figure 2.6). The cold finger and crystal mass was measured again without this portion of the drip. The filed crystal drip portion generally weighed about $0.03 \mathrm{~g}$ and was collected in a 15 $\mathrm{mL}$ vial and analyzed by ICP-MS. This portion of the crystal represented by the double hatch marks in Figure 2.6 was assumed to be only drip, the single hatch marks represents a drip affected region of the purified crystal, and the region with no hatch marks represents the portion that would be only purified $\mathrm{LiCl}$ crystal. A portion of the crystal's edge was now chipped away and dimension "c" could then be measured and was measured. The rest of the crystal was chipped away from the cold finger and crushed in a mortar and pestle to small granules. The crushed crystal granules were then placed in a vial for analysis by ICP-MS. Following each experiment at this point the cold finger was cleaned by scraping salt residue from the cold finger until its mass returned to where it was at the beginning of the 
experiment. The cold finger was also wiped clean with parafilm to remove any salt powder remains. The post processing of purified crystals up to this point required 3 to 4 hours.

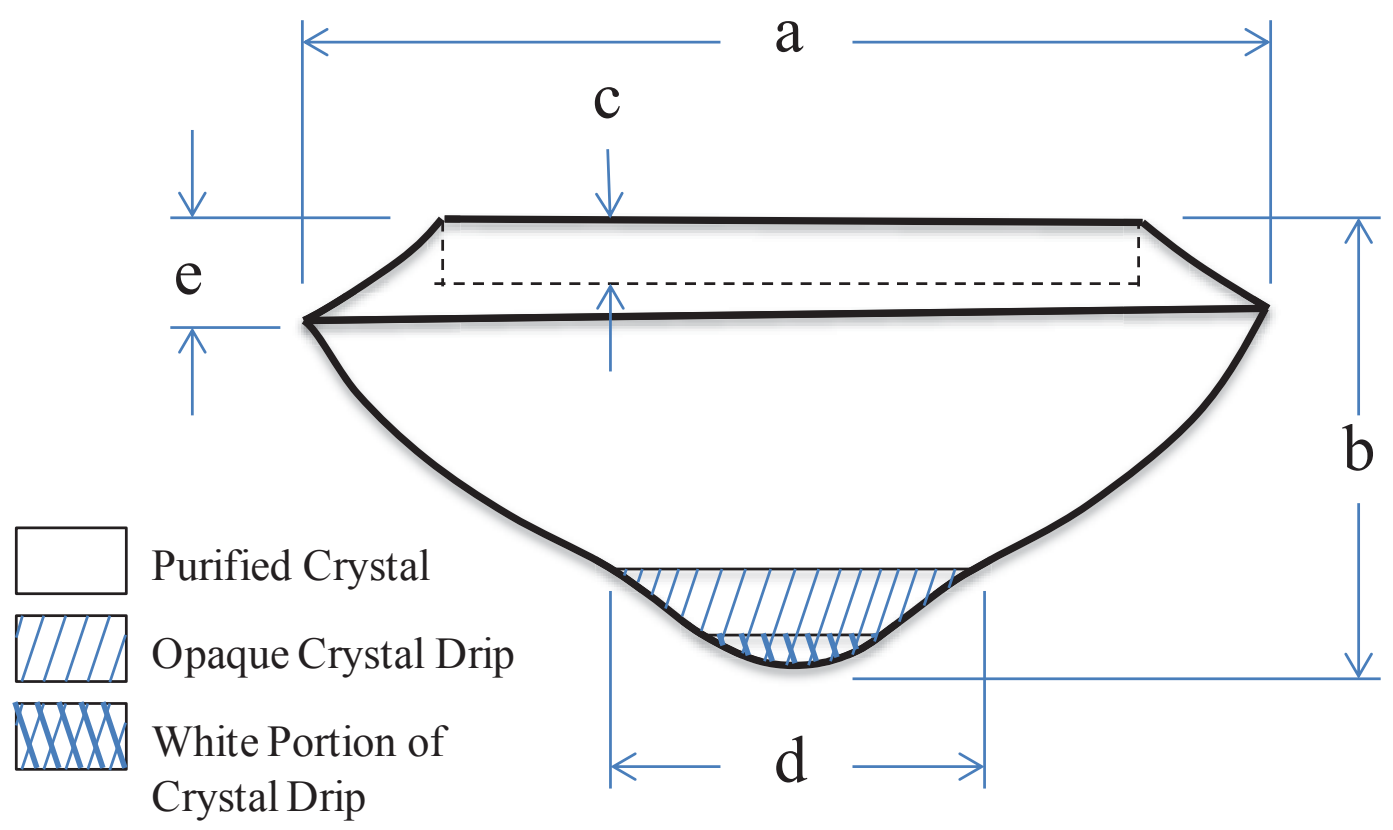

Figure 2.6: Experimental crystal representation showing color regions and dimensions that were measured.

\subsubsection{Sample Analysis and Preparation}

The sample preparation for ICP-MS elemental analysis is outlined and discussed as follows. There were three basic routines used in ICP-MS analysis; they were for (1) the bulk, (2) the drip on the crystal, and (3) the crystal without the drip. The bulk sample came from the $\mathrm{CsCl}$ concentrated bulk salt at the end of experiments. The drip sample was obtained from the drip filings removed from the crystals. The crystal sample was from the remaining crystal after crystal drip had been removed for experiments performed at initial concentrations of 1,3 , and $7.5 \mathrm{wt} \% \mathrm{CsCl}$. For experiments with initial concentration of 5 $\mathrm{wt} \% \mathrm{CsCl}$, the crystal sample came from the entire crystal including the drip. For each sample, the assumption was made that as samples were crushed or filed they were mixed sufficiently to represent the average composition of the total salt volumes. The samples were weighed out and placed in $15 \mathrm{~mL}$ vials. The sample masses were $0.1 \mathrm{~g}$ for both the bulk and 
crystal samples and $0.01 \mathrm{~g}$ to $0.05 \mathrm{~g}$ for the drip samples, depending on the amount of drip that could be removed without destroying the crystal's structural integrity. The samples were digested for more than 12 hours in $3 \mathrm{~mL}$ of nitric acid $\left(\mathrm{HNO}_{3}\right)$. After that, $10 \mathrm{~mL}$ of $18 \mathrm{~m} \Omega$ nanopure water was added to the digested samples, which were then agitated to ensure a homogenous mixture. Then $50 \mu \mathrm{L}$ of the solution was placed in another $15 \mathrm{~mL}$ vial and diluted 241 times by adding $12 \mathrm{~mL}$ of $18 \mathrm{~m} \Omega$ nanopure water. Then $1 \mathrm{~mL}$ of this solution was placed in another $15 \mathrm{~mL}$ vial and was diluted 10 times by adding $9 \mathrm{~mL}$ of $5 \% \mathrm{HNO}_{3}$. This final diluted sample was then analyzed for elemental composition of Li and Cs. Each experiment required a total of 2 to 3 days to complete.

\subsection{Experimental Plan}

Experiments were performed for 5 initial salt compositions of $\mathrm{CsCl}(0,1,3,5$, and 7.5 $\mathrm{wt} \%$ ) to optimize and understand cold finger crystal growth separation (see Table 2.1). Exp. No. 1a was done by varying crystal growth separation time $(1,2,3,4,5,10,15,30$, and 60 min) at a constant cooling gas flow rate of $11.0 \mathrm{~L} / \mathrm{min}$. Exp. No. $1 \mathrm{~b}$ was performed by fixing the crystal growth time at $30 \mathrm{~min}$ and varying the cooling gas flow rate $(5.1,6.2,6.8,7.4,8.5$, 9.8, and $11.0 \mathrm{~L} / \mathrm{min})$. Both experimental runs were performed with pure $\mathrm{LiCl}$. The results of these experiments provided an experimental base line and operational limitations of the experimental setup. The second series of experiments (Exp. No. 4) was with an initial $5 \mathrm{wt} \%$ $\mathrm{CsCl}$ in $\mathrm{LiCl}$ for various separation times $(5,10,15$, and $30 \mathrm{~min})$ and cooling rates $(7.4,9.8$, 12.3, and $14.9 \mathrm{~L} / \mathrm{min}$ ), that provided optimum cold finger separation operating variables. The other series of experiments varied cooling rate $(7.4,9.8,12.3$, and $14.9 \mathrm{~L} / \mathrm{min})$, and showed how crystal drip residue and initial concentration effected purified electrolyte production and purity at a constant crystal growth time of 15 minutes (Exp. Nos. 2, 3, and 5).

From now on, the discussion throughout this work will refer to Table 2.1. To facilitate discussion, a standard notation will be used. This notation specifies the experiment number (\#) and its specific crystal growth time (x) and cooling gas flow rate (y) operating conditions in the form of Exp\#(x, y). For example, Exp4(15, 14.9) refers to the experiment performed with initial $5 \mathrm{wt} \% \mathrm{CsCl}$ at a crystal growth time of $15 \mathrm{~min}$ and cooling gas flow

rate of $14.9 \mathrm{~L} / \mathrm{min}$. For a more detailed list of experiments performed see Appendix A and B, containing raw mass measurements and ICP-MS analysis data, respectively. 
Table 2.1: Experiments performed for this work.

\begin{tabular}{cccc}
\hline Exp. No. & $\begin{array}{c}\text { Initial LiCl-CsCl } \\
\text { Concentration }\left(\mathrm{wt}^{2} \mathrm{CsCl}\right)\end{array}$ & $\begin{array}{c}\text { Crystal Growth Time } \\
(\mathrm{min})\end{array}$ & $\begin{array}{c}\text { Cooling Gas Flow Rate } \\
(\mathrm{L} / \mathrm{min})\end{array}$ \\
\hline $1 \mathrm{a}$ & 0 & $1-60$ & 11.0 \\
\hline $1 \mathrm{~b}$ & 0 & 30 & $5.1-11.0$ \\
\hline 2 & 1 & 15 & $7.4-14.9$ \\
\hline 3 & 3 & 15 & $7.4-14.9$ \\
4 & 5 & $5-30$ & $7.4-14.9$ \\
\hline 5 & 7.5 & 15 & $7.4-14.9$ \\
\hline
\end{tabular}




\section{Chapter 3 Experimental Results and Discussion}

\subsection{Preliminary Experiments with Pure LiCl}

Two series of experiments were conducted using pure $\mathrm{LiCl}$ to provide a base line for crystal growth behavior and to identify experimental setup limitations by determining feasible ranges for operating variables (crystal growth time and cooling gas flow rate). The first series of experiments were conducted at a fixed cooling gas flow rate of $11.0 \mathrm{~L} / \mathrm{min}$ while crystal growth time was varied from 1 to $60 \mathrm{~min}$ (see Figure 3.1). The plotted data in Figure 3.1 shows that the crystal growth rate exhibits exponential growth decay. The fixed cooling gas flow rate was selected as an upper limit to determine a maximum crystal growth time, were the cooling gas flow rate was in the upper region of the flow control range. The maximum crystal growth time was determined based on the geometric limitations of the experimental set up. Photographic representations of the data set in Figure 3.1 reveal that for crystal growth times above 30 min the natural crystal growth geometry begins to be obstructed by the geometric constraints of the molten salt depth $(\sim 12 \mathrm{~mm})$ in the crucible of the experimental setup (see Figure 3.2). As a result, the maximum crystal growth time is 30 min for an upper cooling gas flow rate limit of $11.0 \mathrm{~L} / \mathrm{min}$ for this given geometry. When making reference to crystal growth in this work it refers to freezing. Freezing is a phenomenon of phase change from liquid to solid induced by energy removal from the liquid phase until it thermodynamically favors a solid phase. 


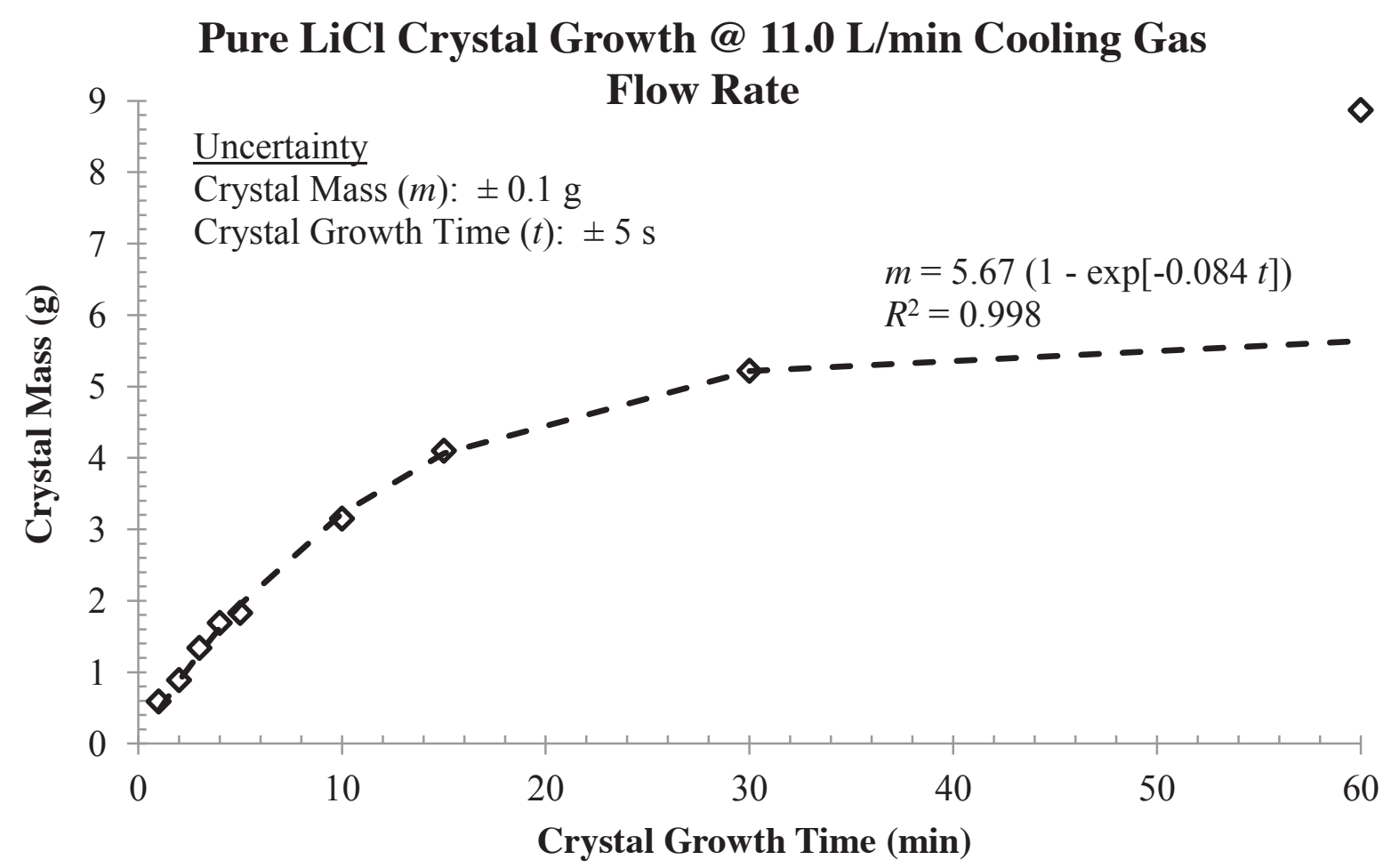

Figure 3.1: Crystal mass vs. crystal growth time for pure $\mathrm{LiCl}$ experiments at an $11.0 \mathrm{~L} / \mathrm{min}$ cooling gas flow rate.

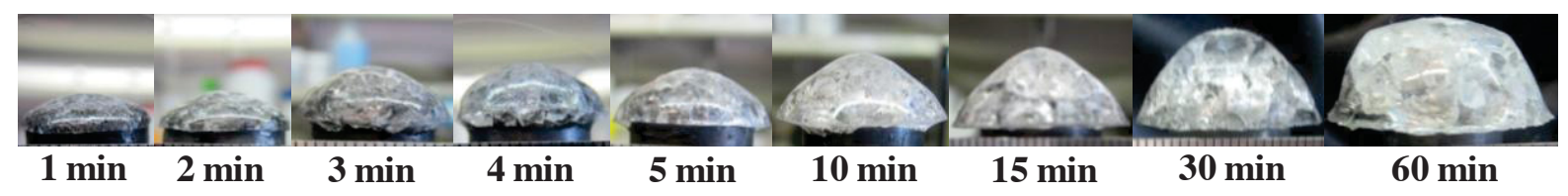

Figure 3.2: A montage of pure $\mathrm{LiCl}$ experiments for varying growth times at an $11.0 \mathrm{~L} / \mathrm{min}$ cooling gas flow rate.

The second experimental set (Exp. No. 1b) was conducted using the maximum crystal growth time of 30 min while varying the cooling gas flow rates, to determine a lower limit for cooling gas flow rate. The cooling gas flow rate was incrementally decreased until an insignificant amount of crystal was formed on the cold finger after $30 \mathrm{~min}$ of crystal growth (see Figure 3.3). The data in Figure 3.3 shows that at a cooling gas flow rate of $5.1 \mathrm{~L} / \mathrm{min}$ the grown crystal mass is approaching an asymptote near zero, indicating the lowest limit for cooling gas flow rate. Photographs of the data plotted in Figure 3.3 give a visual representation of the insignificant amount of crystal formed at a cooling gas flow rate of 5.1 $\mathrm{L} / \mathrm{min}$ (see Figure 3.4). The results of both series of pure $\mathrm{LiCl}$ experiments were used to determine operating variable ranges for experiments using a $\mathrm{LiCl}-\mathrm{CsCl}$ salt system. 


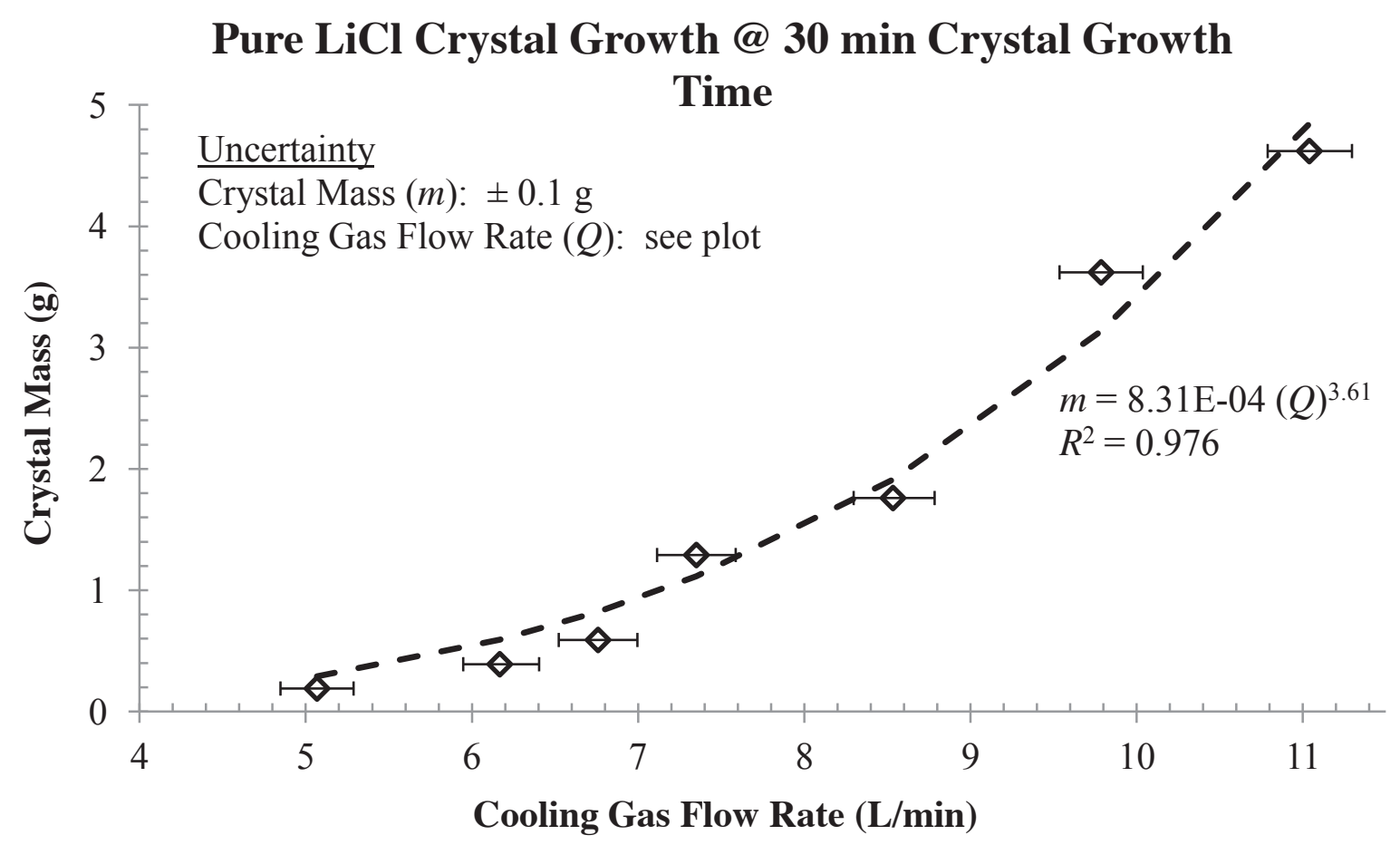

Figure 3.3: Crystal mass vs. cooling gas flow rate for pure $\mathrm{LiCl}$ experiments at a 30 min crystal growth time.

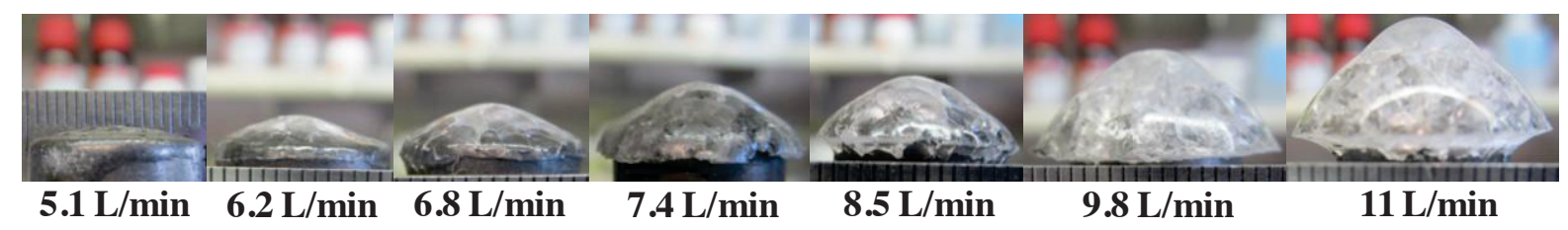

Figure 3.4: A montage of pure $\mathrm{LiCl}$ experiments for varying cooling gas flow rates at a $\mathbf{3 0}$ min crystal growth time.

\subsection{Experiments with Initial $5 \mathrm{wt} \% \mathrm{CsCl}$ in $\mathrm{LiCl}$}

Experiments with an initial $5 \mathrm{wt} \% \mathrm{CsCl}$ in $\mathrm{LiCl}$ were conducted to determine the effects of crystal growth time and cooling gas flow rate on crystal growth rate and purity. The initial $5 \mathrm{wt} \% \mathrm{CsCl}$ concentration in $\mathrm{LiCl}$ was selected as a representative concentration that would be found in electrolytic oxide reduction electrolyte that is due for replacement or purification [5]. The effects of crystal growth time and cooling gas flow rate on crystal growth and purity were analyzed with results from Exp. No. 4 (see Figure 3.5). The variable ranges were selected with the aid of preliminary pure $\mathrm{LiCl}$ experiments in order to produce 
crystal geometry within geometric constraints of experimental setup. The experimental matrix in Figure 3.5 reports $\mathrm{CsCl}$ concentrations as $\mathrm{wt} \% \mathrm{CsCl}$ (located on the upper left hand corner of each image) and shows crystal sizes and shapes. The concentrations reported as wt $\% \mathrm{CsCl}$ were calculated with mole balances using raw ICP-MS data.

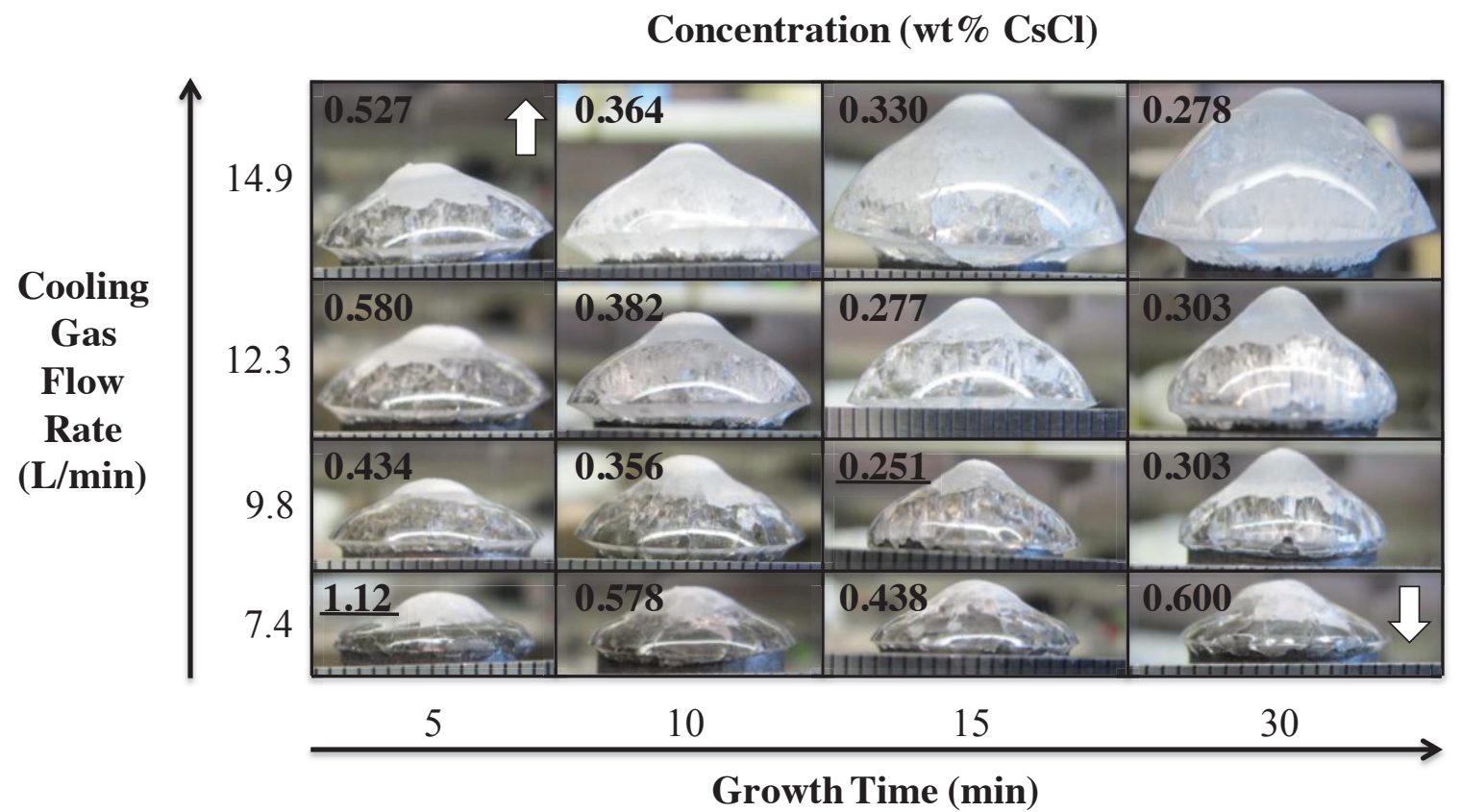

Figure 3.5: Crystal concentration results for experiments performed at an initial bulk concentration of 5 wt \% CsCl in LiCl.

Figure 3.5 shows both assumed and experimental highest and lowest concentrations with white arrows and underlined data. Here, assumed concentrations were based on ideal assumptions for thermodynamic equilibrium before experiments were performed and compared with actual results of experiments. The grown crystal mass was assumed to increase as both crystal growth time and cooling gas flow rate increase. The highest $\mathrm{CsCl}$ concentration for purified crystals was assumed to be the experimental crystal with the shortest growth time and largest cooling gas flow rate indicated with a white arrow pointing upward in Figure 3.5. The lowest $\mathrm{CsCl}$ concentration for purified crystals was assumed to be the experimental crystal with the longest crystal growth time and smallest cooling gas flow rate indicated with a white arrow pointing downward in Figure 3.5. The experimental crystal growth rate matched assumed results, but the experimental purified crystal concentration did 
not match assumed results. The theory of phase equilibrium as it relates to crystal purity guided the intuition of crystal concentrations. The theory states that (1) if a salt system is at equilibrium throughout an entire experiment the crystal would be $100 \%$ pure and (2) if forced from equilibrium by heat removal the salt system impurities would freeze with pure species before the system could return to an equilibrium state. The impure and pure species freeze together because the heat removal occurs faster than the diffusion of impure species away from pure species, so higher cooling rates would result in higher impurity concentrations. Contrary to the assumed crystal concentrations, the highest $\mathrm{CsCl}$ concentration was for the experimental crystal with the shortest growth time and smallest cooling gas flow rate $(\operatorname{Exp} 4(5,7.4))$ and the lowest $\mathrm{CsCl}$ concentration was for $\operatorname{Exp} 4(15,9.8)$ shown as bolded data in Figure 3.5.

Detailed observation of the crystal formations reveals a potential explanation for why the experimental crystal concentration results are different from the expected results. The assumption with the expected guess was based on the following two reasons: (1) the crystal was formed only while submerged in the molten salt during cold finger operation and (2) there was no bulk salt residue on the crystal when removed from the molten salt. Here, it was discovered that crystal mass formed during cold finger operation was transparent and crystal formed during a more rapid cool down was opaque. An examination of each crystal showed that a portion of the crystal, shaped in the form of a drip, was opaque indicating that bulk salt residue was removed with crystals (see Figure 3.6). Figure 3.6 shows a side and bottom view of a pure $\mathrm{LiCl}$ and $\mathrm{LiCl}-\mathrm{CsCl}$ experiment at a common growth time of $30 \mathrm{~min}$ and a cooling gas flow rate of $11.0 \mathrm{~L} / \mathrm{min}$. The figure includes black lines highlighting the opaque residue drip formation. The most likely cause for the opaque coloring of the drip is that it freezes faster than the rest of the crystal formed during normal cold finger operation, creating more grain boundaries in its formation.

A potential explanation for the unexpected concentrations is that the drip was formed with $\mathrm{CsCl}$ concentrated bulk salt that froze to the crystal as the cold finger and crystal were removed from the concentrated molten salt. This explains why the smaller crystals have higher $\mathrm{CsCl}$ concentrations; because the mass fractions of the $\mathrm{CsCl}$ concentrated drip is large for smaller crystals and smaller for larger crystals. It can be seen that the crystal mass for 
$\mathrm{LiCl}-\mathrm{CsCl}$ (initial $5 \mathrm{wt} \% \mathrm{CsCl}$ ) decreases by one half the crystal mass for pure $\mathrm{LiCl}$ at the same cold finger operating conditions and parameters (see Figure 3.6). This change in mass is due to the diffusive and convective effects of heat and mass transfers. The heat transfer between the pure $\mathrm{LiCl}$ and $\mathrm{LiCl}-\mathrm{CsCl}$ experiments would be similar, suggesting that the primary reason for the decrease in crystal mass for the $\mathrm{LiCl}-\mathrm{CsCl}$ experiment is a lower melting point at the crystal surface affected by mass transfer as $\mathrm{CsCl}$ moves into the bulk via convection and diffusion.

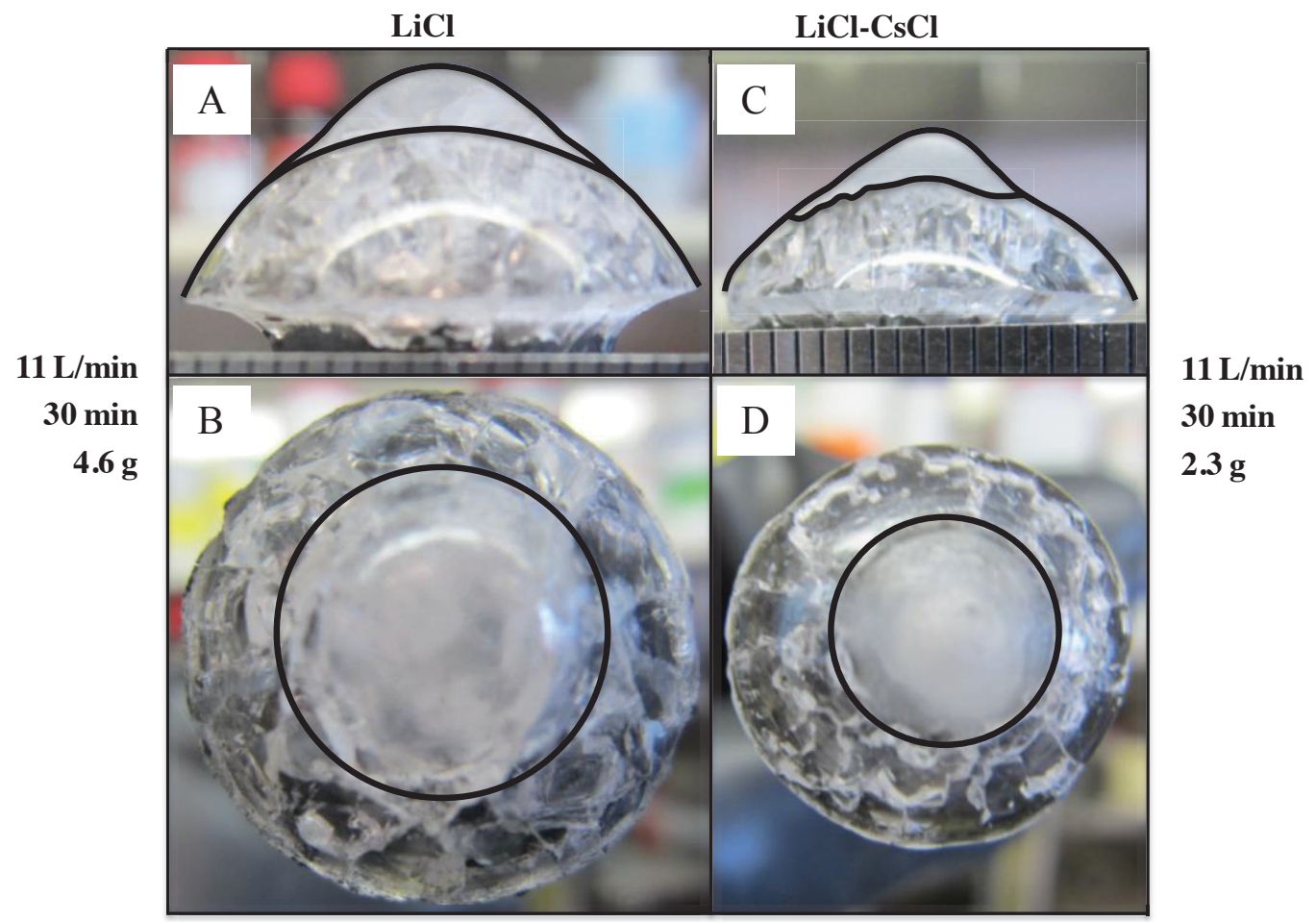

Figure 3.6: Crystal mass comparison for pure $\mathrm{LiCl}(\mathrm{A}$ and $\mathrm{B}$ ) and $\mathrm{LiCl}$ with an initial 5 wt \% $\mathrm{CsCl}$ (C and $\mathrm{D}$ ) at the same cold finger operating conditions and parameters with lines highlighting an opaque residue drip formation.

The crystal growth rate for both pure $\mathrm{LiCl}$ and $\mathrm{LiCl}-\mathrm{CsCl}$ experiments were analyzed and compared with each other to explore potential production rates using cold finger melt crystallization separation. The crystal and drip mass data for pure $\mathrm{LiCl}$ and $\mathrm{LiCl}-\mathrm{CsCl}$ with 5 $\mathrm{wt} \% \mathrm{CsCl}$ experiments were plotted versus growth time, to study the effect both cooling rate and initial $\mathrm{CsCl}$ concentration have on crystal growth rate (see Figure 3.7). As expected the mass versus time plot shows an increase in crystal mass as crystal growth time increases. Each of the mass profiles follows uniform and predictable exponential trends that increase 
linearly in magnitude with increasing cooling gas flow rate. The mass profiles for $5 \mathrm{wt} \%$ $\mathrm{CsCl}$ experiments at 7.4, 9.8, and $12.3 \mathrm{~L} / \mathrm{min}$ cooling gas flow rates all appear to reach a steady state crystal and drip mass within 30 min of crystal growth time. The crystal and drip data series for pure $\mathrm{LiCl}$ includes masses for crystal growth times ranging from 1 to $60 \mathrm{~min}$, which gives an idea of projected mass versus time profiles for experiments with $5 \mathrm{wt} \% \mathrm{CsCl}$ in $\mathrm{LiCl}$. For each mass versus time profile the mass is zero when crystal growth time is zero. Figure 3.7 also provides a graphical representation of the two experiments that are presented in Figure 3.6 for pure $\mathrm{LiCl}$ and $\mathrm{LiCl}-\mathrm{CsCl}$ with crystal growth times at $30 \mathrm{~min}$ and cooling gas flow rates at $11.0 \mathrm{~L} / \mathrm{min}$.

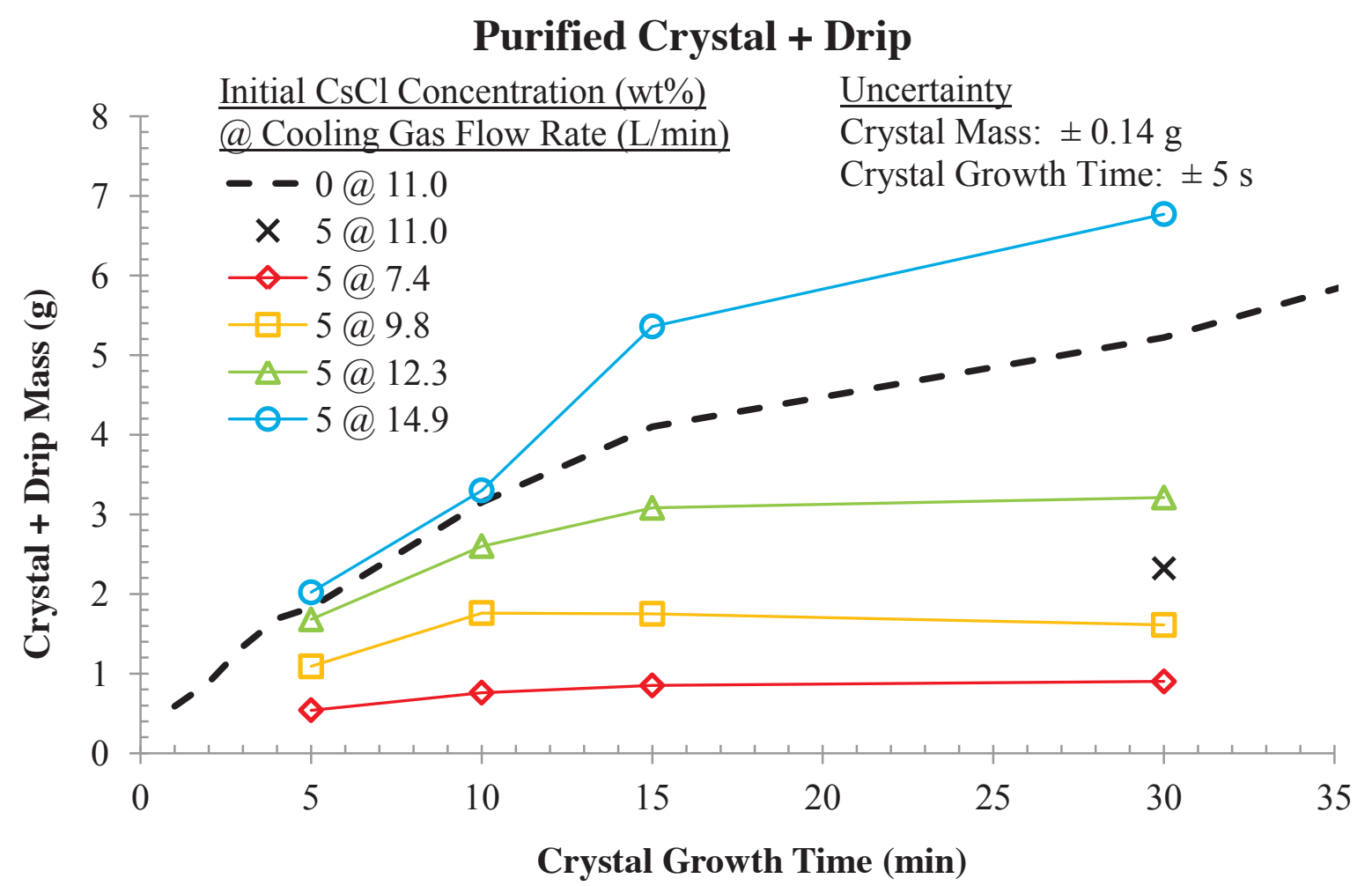

Figure 3.7: Mass vs. time for crystal and drip with an initial $5 \mathrm{wt} \% \mathrm{CsCl}$ in $\mathrm{LiCl}$ for varying cooling gas flow rates compared to pure $\mathrm{LiCl}$ experiments at a cooling gas flow rate of $11.0 \mathrm{~L} / \mathrm{min}$.

The concentration data for initial $5 \mathrm{wt} \% \mathrm{CsCl}$ in $\mathrm{LiCl}$ experiments were analyzed to discover optimum cold finger crystal growth operating parameters. The optimum crystal growth time will be the time that results in the lowest $\mathrm{CsCl}$ concentration representing the best crystal purity. A plot of crystal and drip concentration versus crystal growth time showed a $\mathrm{CsCl}$ concentration minimum at a growth time of $15 \mathrm{~min}$ for three of the four 
cooling gas flow rate data series (see Figure 3.8). The other data series had a minimum $\mathrm{CsCl}$ concentration at a $30 \mathrm{~min}$ growth time. However, it is possible within uncertainty to have a minimum at a $15 \mathrm{~min}$ growth time for this data series. So within uncertainty, ranging from \pm 0.039 to $\pm 0.074 \mathrm{wt} \% \mathrm{CsCl}$, the optimum growth time is $15 \mathrm{~min}$ for the cooling rate data sets in Figure 3.8. Now that an optimum crystal growth time of $15 \mathrm{~min}$ has been selected, an optimum cooling gas flow rate can now be selected with this crystal growth time optimum.

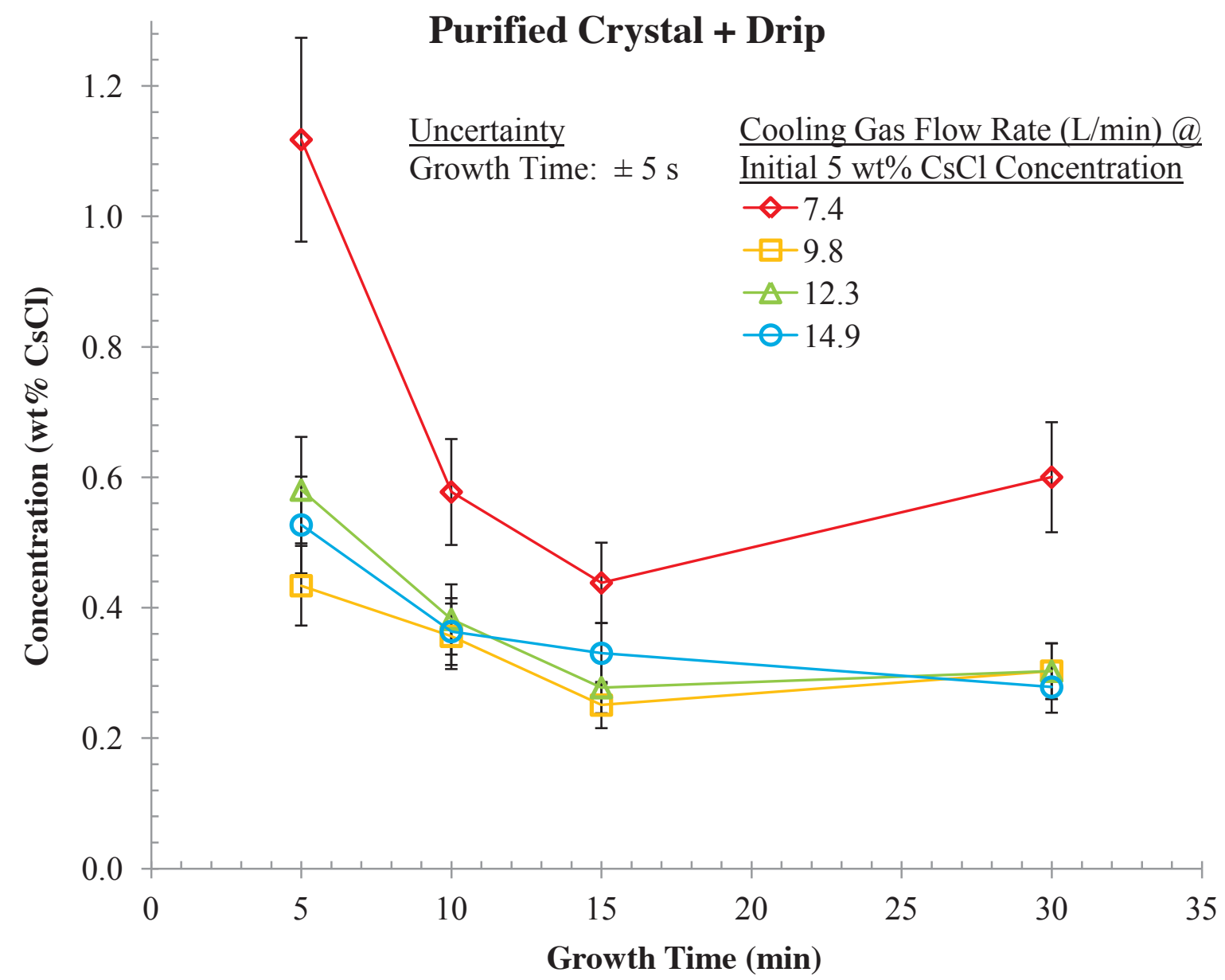

Figure 3.8: Purified crystal and drip concentration vs. crystal growth time for initial $5 \mathrm{wt} \% \mathrm{CsCl}$ in $\mathrm{LiCl}$ for varying cooling gas flow rates.

To select an optimum cooling gas flow rate, both crystal purity and production rate must be considered to make a well rounded economical selection. Both these parameters were analyzed with a plot of crystal and drip concentration versus mass growth rate (see Figure 3.9). The plot shows that there are two potential optimum values for cooling gas flow rate determined by whether separation purity or separation production rate is more important. 
If separation purity is more important, the optimum cooling gas flow rate would be the data point with the lowest $\mathrm{CsCl}$ concentration $(\operatorname{Exp} 4(15,9.8))$ producing $0.12 \mathrm{~g} / \mathrm{min}$ at $0.25 \mathrm{wt} \%$ $\mathrm{CsCl}$. If separation production rate is more important, the optimum cooling gas flow rate would be the data point with the highest crystal growth rate $(\operatorname{Exp} 4(15,14.9))$ producing 0.36 $\mathrm{g} / \mathrm{min}$ at $0.33 \mathrm{wt} \% \mathrm{CsCl}$. Assuming separation production rate is more important than separation purity due to the relatively low $\mathrm{CsCl}$ concentrations of data at optimum crystal growth time of $15 \mathrm{~min}$, the optimum cooling gas flow rate was selected to be $14.9 \mathrm{~L} / \mathrm{min}$.

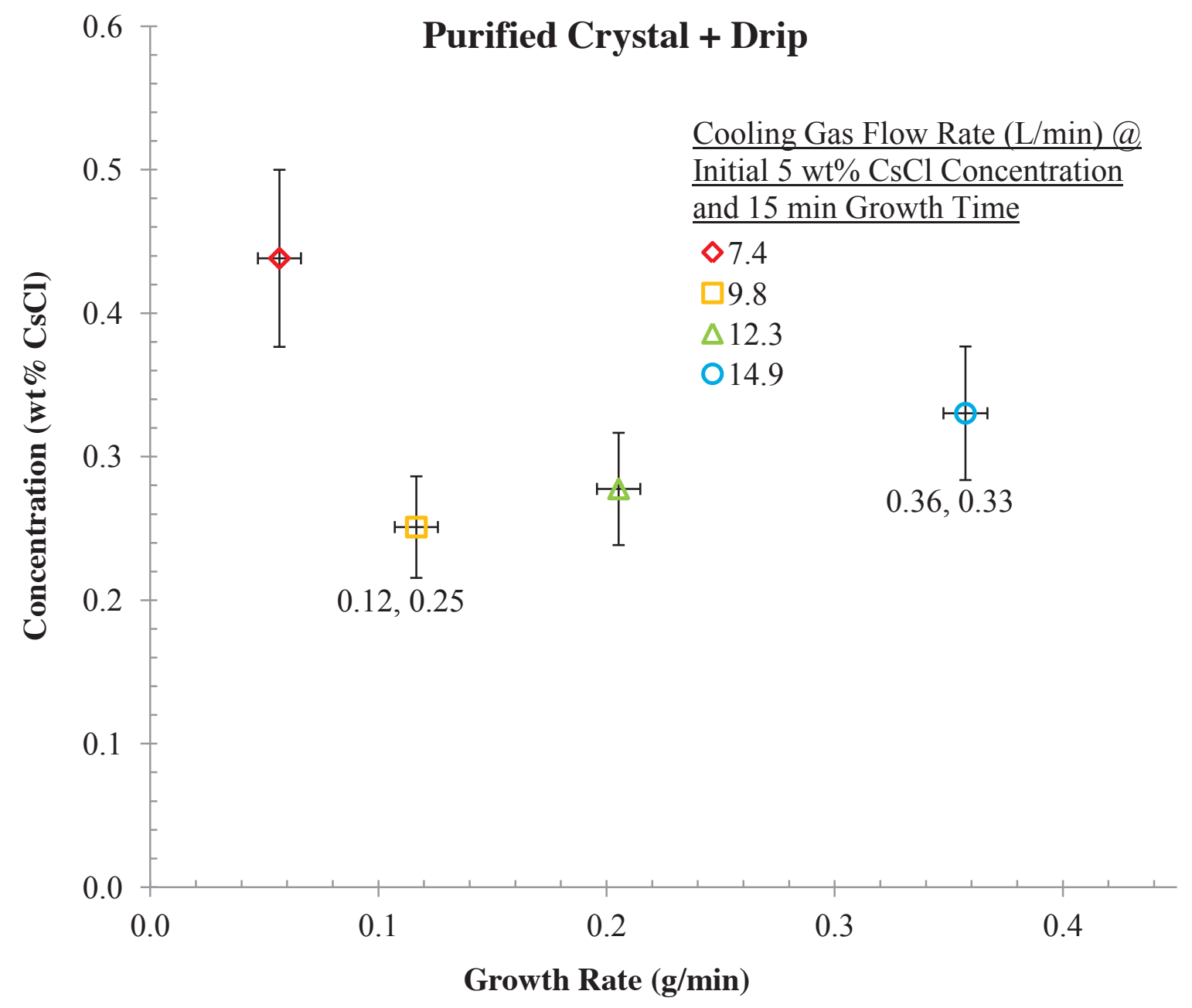

Figure 3.9: Purified crystal and drip concentration vs. crystal growth rate for initial $5 \mathrm{wt} \% \mathrm{CsCl}$ in $\mathrm{LiCl}$ and 15 min crystal growth times for varying cooling gas flow rates. 


\subsection{Experiments with Initial 1, 3, and $7.5 \mathrm{wt} \% \mathrm{CsCl}$ in $\mathrm{LiCl}$}

Experiments were performed with initial 1,3, and $7.5 \mathrm{wt} \% \mathrm{CsCl}$ in $\mathrm{LiCl}$ to explore how $\mathrm{CsCl}$ concentrated crystal drip formation effects purified crystal concentration and how initial $\mathrm{CsCl}$ concentration of molten salt effects crystal growth rate and purity. The concentrated crystal drip formation was assumed to be approximately the same concentration as the concentrated bulk and a plot of crystal drip versus concentrated bulk supports this assumption (see Figure 3.10). The plot shows that some crystal drip concentrations are higher and some lower than the concentrated bulk concentration. The diagonal line in the plot represents equality between drip and bulk concentrations; that is, the data that lie above the line indicates that the drip has a higher $\mathrm{CsCl}$ concentration than the bulk and vice versa. A potential explanation for data that lie below the line is in the procedure for removing the crystal drip. The drip was filed off and it is likely that a portion of the purified crystal was removed with the drip which would lower the drip sample $\mathrm{CsCl}$ concentration. A potential explanation for data that lies above the line is due to a diffusion boundary layer at the solid liquid interface of the crystal during cold finger operation. When the purified crystal is removed from the concentrated bulk, the diffusion boundary layer contained in a thin film is removed with the crystal. Thus, it freezes to the purified crystal surface before it can drip away from the cooling crystal. Within the diffusion boundary layer, there must be a higher concentration of $\mathrm{CsCl}$ at the crystal's surface than the bulk, otherwise there would be no concentration gradient resulting in no separation. The freezing of this diffusion boundary layer with a higher $\mathrm{CsCl}$ concentration would cause the drip concentration to be higher than the bulk concentration [18]. This diffusion boundary layer concentration would apply to all experimental crystals suggesting that the $\mathrm{CsCl}$ concentration of the drips should all be greater than the bulk. This would indicate that the drip removal process is the main reason for variance between crystal drip concentrations relative to the bulk concentrations. 


\section{Concentrated Drip vs. Bulk}

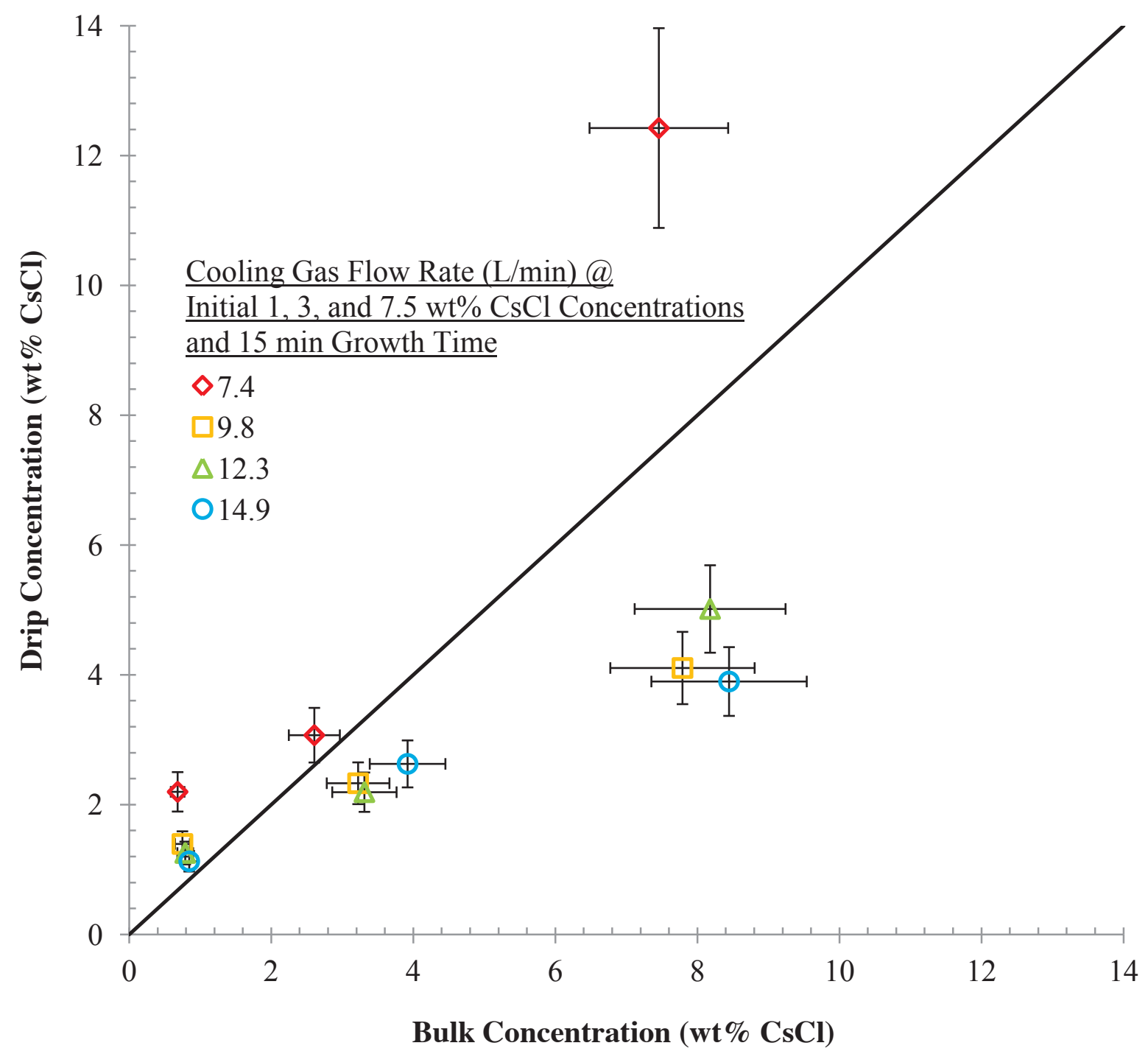

Figure 3.10: Drip concentration vs. bulk concentration for experiments performed at initial concentrations of 1,3 , and $7.5 \mathrm{wt} \% \mathrm{CsCl}$ in $\mathrm{LiCl}$ with 15 min crystal growth times for varying cooling gas flow rates.

Using data from experiments with initial bulk concentrations of $1,3,5$, and $7.5 \mathrm{wt} \%$ $\mathrm{CsCl}$ in $\mathrm{LiCl}$, the effects of initial bulk concentration on crystal growth rate and purified crystal and drip concentration were explored. A plot of crystal and drip mass versus initial bulk concentration shows that crystal and drip mass decrease as initial bulk $\mathrm{CsCl}$ concentration increases for all cooling gas flow rates with crystal growth times of $15 \mathrm{~min}$ (see Figure 3.11). Figure 3.12 shows a plot of purified crystal and drip concentration versus initial bulk concentration. The results reveal that as initial bulk $\mathrm{CsCl}$ concentration increases, 
purified crystal and drip concentration increases by less than $0.5 \mathrm{wt} \% \mathrm{CsCl}$ for the initial bulk concentration range of 1 to $7.5 \mathrm{wt} \% \mathrm{CsCl}$ with the exception of one data point. The data point that has a crystal and drip concentration increase larger than $0.5 \mathrm{wt} \% \mathrm{CsCl}$ is $\mathrm{Exp} 5(15$, 7.4). The larger concentration of this data point is because it has the smallest crystal and drip mass as shown in Figure 3.11 and would have the most unstable crystal and drip concentration. The instability is because the drip mass fraction would be largest for this data point and the drip's $\mathrm{CsCl}$ concentration would be relatively higher than other experiments ran at initial $\mathrm{CsCl}$ concentrations lower than $7.5 \mathrm{wt} \% \mathrm{CsCl}$. With this discussion, it is safe to say that initial bulk concentration has a relatively small effect on crystal growth purity within an initial bulk concentration range of 1 to $7.5 \mathrm{wt} \% \mathrm{CsCl}$ in $\mathrm{LiCl}$.

\section{Purified Crystal + Drip}

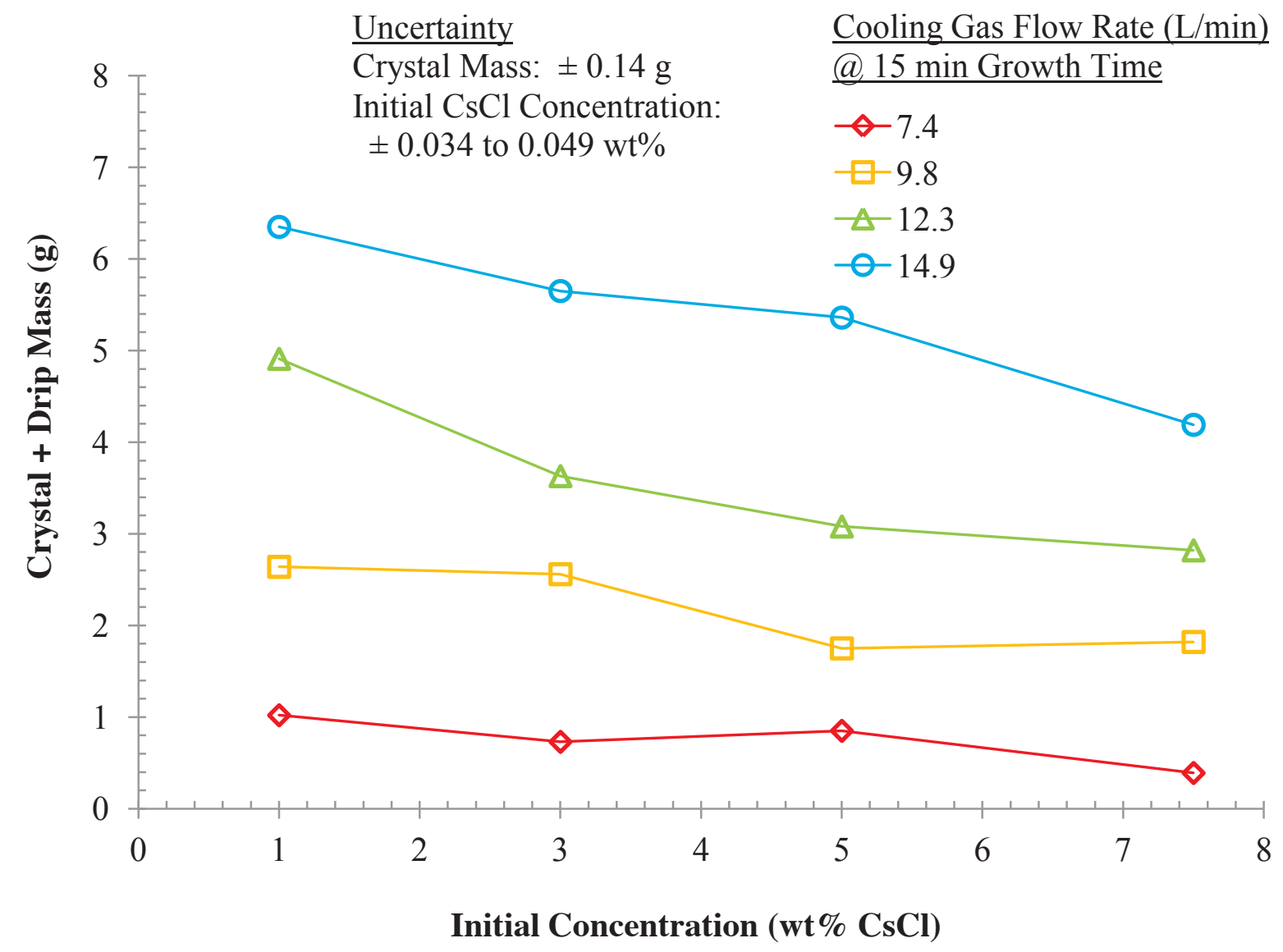

Figure 3.11: Purified crystal and drip mass vs. initial bulk concentration for experiments performed with 15 min crystal growth times for varying cooling gas flow rates. 


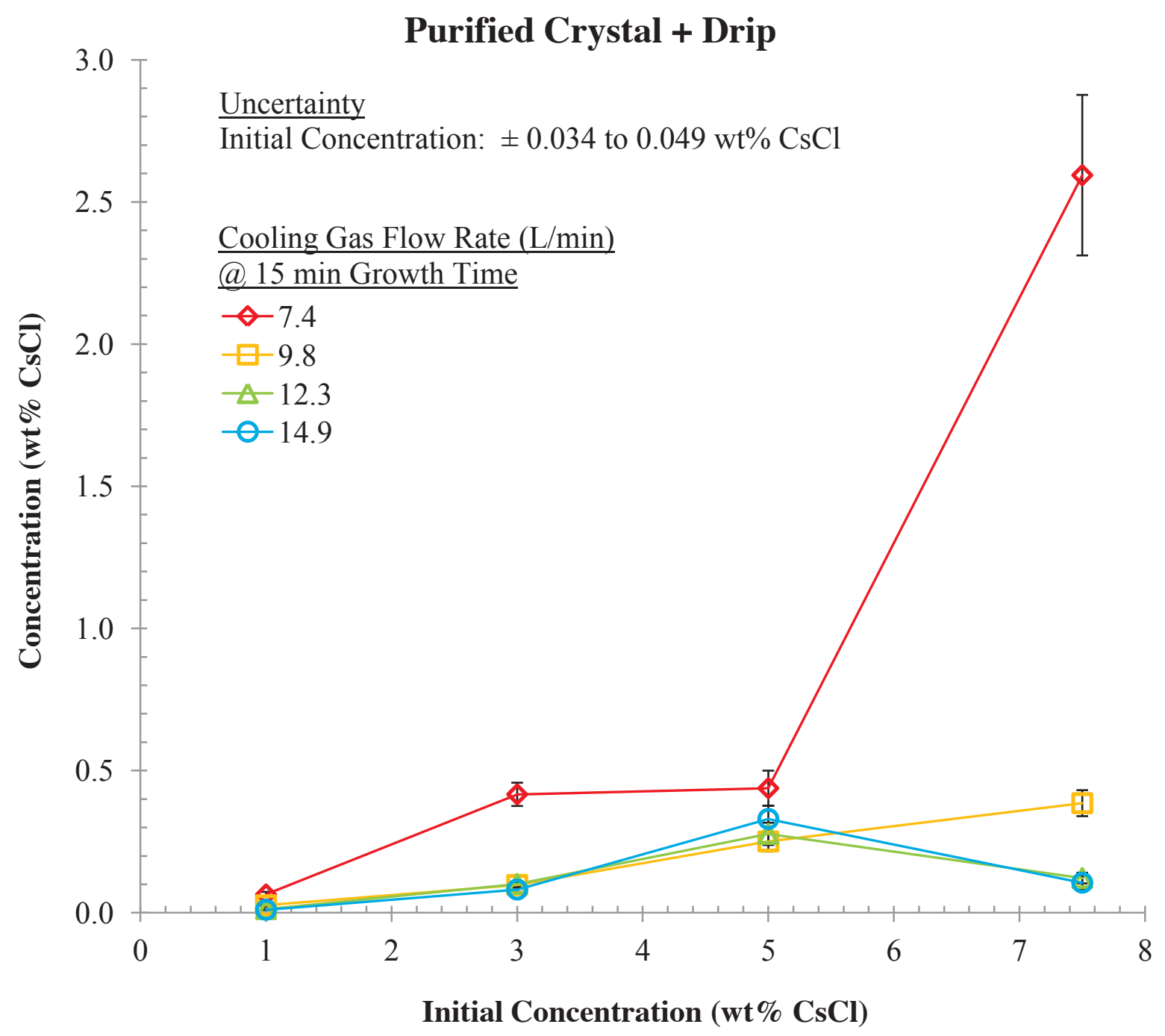

Figure 3.12: Purified crystal and drip concentration vs. initial bulk concentration for experiments performed with 15 min crystal growth times for varying cooling gas flow rates.

\subsection{Potential Scale-up and Application}

A potential scale-up scenario for cold finger melt crystallization separation was applied to an existing bench scale oxide reduction laboratory setup at INL to explore potential purified electrolyte production rates. This bench scale oxide reduction laboratory setup was used to process used nuclear fuel. The used nuclear fuel was placed in a $700 \mathrm{~g}$ batch of $\mathrm{LiCl}-\mathrm{Li}_{2} \mathrm{O}$ electrolyte with $1 \mathrm{wt} \% \mathrm{Li}_{2} \mathrm{O}$ in a $\mathrm{MgO}$ crucible that measured $10 \mathrm{~cm}$ in diameter and $11 \mathrm{~cm}$ in height [5]. The oxide reduction anode and cathode assembly is 
removable so a cold finger assembly can be used to purify a batch of used electrolyte while the oxide reduction process is off line and removed from the crucible. The maximum number of cold fingers that could fit in the crucible without changing the natural crystal growth shape is seven (see Figure 3.13). Figure 3.13 includes a top view looking into the crucible with a potential cold finger arrangement. The white ring around each cold finger represents the space occupied by the purified crystal. The scale-up scenario of this work was designed to concentrate a $700 \mathrm{~g}$ batch of used $\mathrm{LiCl}-\mathrm{CsCl}$ electrolyte from 5 to $7.5 \mathrm{wt} \% \mathrm{CsCl}$ with 7 cold fingers operating at the aforementioned optimum crystal growth time of $15 \mathrm{~min}$ and cooling gas flow rate of $14.9 \mathrm{~L} / \mathrm{min}$. The electrolyte was concentrated to $7.5 \mathrm{wt} \% \mathrm{CsCl}$ so that the effects of initial $\mathrm{CsCl}$ concentration could be accounted for by interpolating purified crystal mass and concentration values for each crystal growth cycle. It would require 7 growth cycles with an approximate total operation time of $105 \mathrm{~min}$ to concentrate used electrolyte from 5 to $7.5 \mathrm{wt} \% \mathrm{CsCl}$. Given this scale-up scenario, a potential purified electrolyte production rate of $136 \mathrm{~g} / \mathrm{hr}$ was calculated with an associated purity calculation of $0.24 \mathrm{wt} \% \mathrm{CsCl}$.

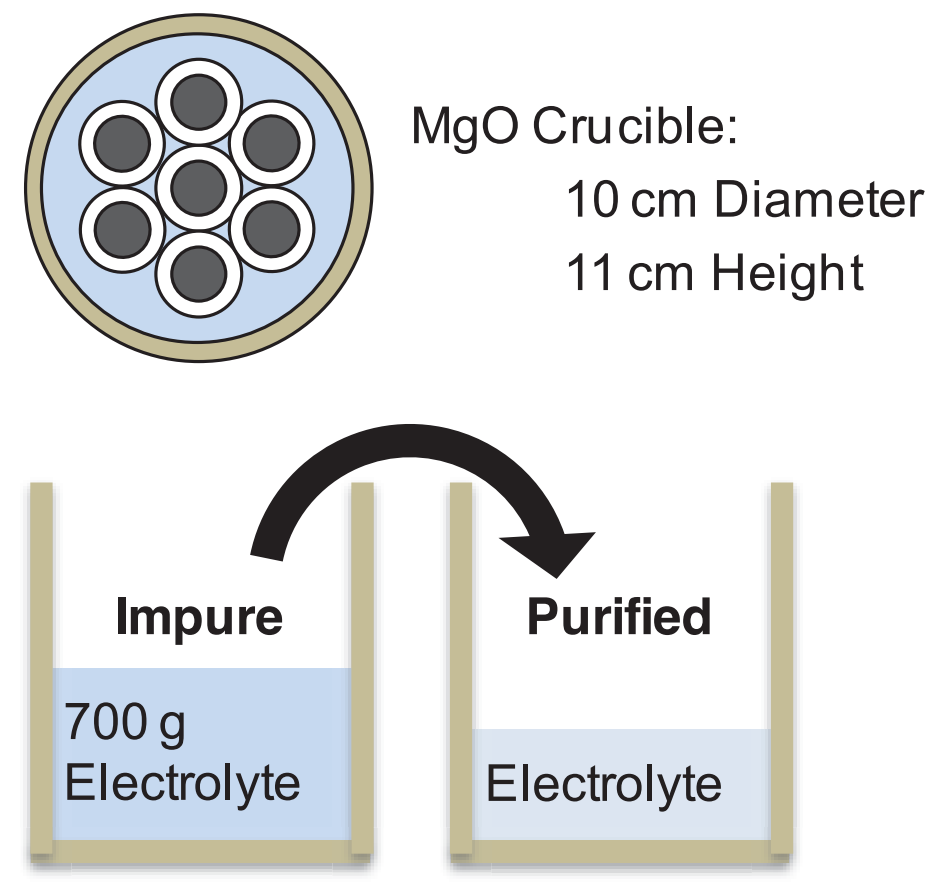

Figure 3.13: Scale-up scenario and application of cold finger melt crystallization with existing bench scale oxide reduction equipment at INL [5]. 


\subsection{Crystal Growth Rate Correlations}

Both heat and mass transfers affect growth rate through convection and a form of diffusion for any crystal growth process. Here, the system is transient with four independent variables (temperature, concentration, position, and time) and is correlated through different dimensionless groups. Transient systems of equations for systems including heat and mass transfer are difficult to solve but with the use of dimensionless groups the crystal growth rate can be correlated with system variables.

The dimensionless groups of the crystal growth rate correlations are derived from dimensional analysis, to generalize the systems' correlations to apply to similar melt crystallization systems. Dimensional analysis is a method of determining dimensionless groups consisting of parameters that describe a system of interest. Any individual dimensionless group of a system is a function of the other dimensionless groups of that system [19]. After obtaining proper dimensionless groups, the groups are used to formulate correlations that are fitted to given data sets to obtain unknown coefficients and/or exponent powers of the correlations. Common dimensionless groups for heat transfer in a natural convective system like cold finger crystal growth are Biot (Bi), Fourier (Fo), Jakob ( $\mathrm{Ja}$ ), Nusselt (Nu), Grashof ( $G r)$, and Prandtl (Pr) numbers while $G r$, Sherwood (Sh), and Schmidt $(S c)$ numbers are the common dimensionless groups for natural convective mass transfer and the Lewis number $(L e)$ relates heat and mass transfer together. The dimensionless groups based on the crystal growth system in this work are defined as follows:

$$
B i=\frac{h_{t h} L}{k_{c}}=\frac{\text { Internal thermal resistance }}{\text { Surface thermal resistance }}
$$

where $h_{t h}$ is the heat transfer coefficient, $L$ is the characteristic length defined as the cuberoot of purified crystal volume (that is, $L=V^{1 / 3}$ ), and $k_{c}$ is the thermal conductivity of the purified crystal, approximated as pure $\mathrm{LiCl}$ [20]. The characteristic length is based on a length scale that directly relates to heat and mass transfers to provide a relative change as both heat and mass transfer change.

$$
F o=\frac{k t}{\rho c_{P} L^{2}}=\frac{\alpha t}{L^{2}}=\frac{\text { Physical time }}{\text { Thermal diffusion time }}
$$


where $k$ is the average thermal conductivity at the average temperature $\left(T_{A v g}\right)$ of the molten salt between the bulk and solid/liquid interface of the purified crystal, approximated as pure $\mathrm{LiCl}[21], t$ is the crystal growth time, $\rho$ is the average density at $T_{A v g}$ of the molten salt between the bulk and solid/liquid interface of the purified crystal, approximated as pure $\mathrm{LiCl}$, $c_{P}$ is the average specific heat capacity at $T_{A v g}$ of the molten salt at constant pressure between the bulk and solid/liquid interface of the purified crystal, approximated as pure $\mathrm{LiCl}$, and $\alpha$ is the thermal diffusivity at $T_{A v g}$ of the molten salt at constant pressure between the bulk and solid/liquid interface of the purified crystal, approximated as pure $\mathrm{LiCl}$. The average temperature in Kelvin is defined as: $T_{\text {Avg }}=\left(T_{\text {Freeze }}+T_{\text {Bulk }}\right) / 2$ where $T_{\text {Freeze }}$ is the freezing temperature of $\mathrm{LiCl}-\mathrm{CsCl}$ obtained from a phase diagram for given $\mathrm{LiCl}$ mole fractions [16] and $T_{B u l k}$ is the measured molten salt temperature.

$$
J a=\frac{c_{P c}\left(T_{\text {Freeze }}-T_{\text {Bulk }}\right)}{h_{c f}}=\frac{\text { Sensible energy }}{\text { Latent energy }}
$$

where $c_{P c}$ is the specific heat capacity of the purified crystal, approximated as pure $\mathrm{LiCl}$ and $h_{c f}$ is the latent heat of fusion for the purified crystal, approximated as pure $\mathrm{LiCl}$ [22]. Next,

$$
\begin{aligned}
& N u=\frac{h_{t h} L}{k}=\frac{\text { Convection heat transfer }}{\text { Conduction heat transfer }} \\
& G r=\frac{g \beta|\Delta T| L^{3} \rho^{2}}{\mu^{2}}=\frac{\text { Buoyancy force }}{\text { Viscous force }}
\end{aligned}
$$

where $g$ is the gravitational constant $\left(9.81 \mathrm{~m} / \mathrm{s}^{2}\right), \beta$ is the thermal expansion coefficient defined as the negative of the partial derivative of density with respect to temperature at constant pressure divided by the average density of the molten salt $\left(\beta=-(\partial \rho / \partial T)_{p} / \rho\right)[23], \Delta T$ is the temperature difference between the bulk and solid/liquid interface of the purified crystal $\left(\Delta T=T_{\text {Bulk }}-T_{\text {Freeze }}\right)$, and $\mu$ is the average dynamic viscosity at $T_{A v g}$ of the molten salt between the bulk and solid/liquid interface of the purified crystal, approximated as pure LiCl. Here, $\operatorname{Pr}$ is defined as

$$
\operatorname{Pr}=\frac{\mu c_{P}}{k}=\frac{\text { Viscous diffusion }}{\text { Thermal diffusion }}
$$

Then, 


$$
S h=\frac{h_{m} L}{D_{A B}}=\frac{\text { Overall mass diffusion }}{\text { Species diffusion }}
$$

where $h_{m}$ is the mass transfer coefficient and $D_{A B}$ is the species diffusion coefficient of $\mathrm{CsCl}$ into LiCl. Also,

$$
S c=\frac{\mu}{\rho D_{A B}}=\frac{\text { Viscous diffusion }}{\text { Species diffusion }}
$$

Lastly, $L e$ is defined as

$$
L e=\frac{S c}{P r}=\frac{\alpha}{D_{A B}}=\frac{\text { Thermal diffusion }}{\text { Species diffusion }}
$$

The physical equations used to calculate material properties in Eqs. (3.1) - (3.9) are given in Table 3.1, and a complete list of calculated parameters of the dimensionless groups can be found in Appendix C. Experimental values of $\mathrm{Bi}, \mathrm{Fo}, \mathrm{Ja}, \mathrm{Gr}, \mathrm{Pr}, \mathrm{Sc}$, and $\mathrm{Le}$ were calculated on average to be on the order of $0.39,2.8,0.099,71,000,3.5,390$, and 0.009 , respectively, indicating that (1) lumped capacitance cannot be used to model the system because the internal thermal resistance is not sufficiently smaller than the surface thermal resistance (that is, Bi must be less than 0.1 in order to use lumped capacitance with small error by assuming uniform temperature of the solid crystal [23]), (2) experimental crystal growth times allow sufficient time for thermal diffusion to take place, (3) sensible energy is less than latent energy indicating a thermodynamic equilibrium upon phase change, (4) buoyant forces are larger than viscous forces, (5) viscous heat diffusion is dominant over heat conduction, (6) viscous mass diffusion is dominant over species diffusion in the molten salt system, and (7) species diffusion dominates thermal diffusion suggesting that the species diffusion boundary layer is thicker than the thermal diffusion boundary layer [23]. A complete list of calculated $G r, P r$, and $S c$ can be found in Appendix D.

The $N u$ and $S h$ are analogous for heat and mass transfer, respectively, along with $P r$ and $S c$. For an isothermal condition, it has been reported that $N u=f(G r, P r)$ and $S h=$ $f(G r, S c)$ [23]. If the condition is not isothermal and there are gradients in both temperature and concentration, which is the case for cold finger crystal growth separation, heat and mass transfer will occur concurrently by natural convection and would be more accurately described with either $N u=f(G r, P r, S c)$ or $S h=f(G r, S c, P r)$ [23]. It is not acceptable to 
directly substitute $P r$ with $S c$ and say that $N u=S h$ because the diffusion boundary layers were so different. However, it is acceptable to say that $N u$ and $S h$ would have the same family of curves that correlate to experimental data.

Table 3.1: Equations used to calculate molten salt properties at $\boldsymbol{T}_{\text {Avg }}$.

\begin{tabular}{llcc}
\hline \multicolumn{1}{c}{ Variable } & \multicolumn{1}{c}{ Equation } & Units & References \\
\hline Average density & $\rho=1.88 \mathrm{E}+3-0.433 \times T_{A v g}$ & $\mathrm{~kg} / \mathrm{m}^{3}$ & {$[17]$} \\
$\begin{array}{l}\text { Average dynamic } \\
\text { viscosity }\end{array}$ & $\mu=1.09 \mathrm{E}-4 \times \exp \left[1.91 \mathrm{E}+4 /\left(8.31 \times T_{A v g}\right)\right]$ & $\mathrm{kg} /(\mathrm{s} \mathrm{m})$ & {$[17]$} \\
$\begin{array}{l}\text { Average specific } \\
\text { heat capacity }\end{array}$ & $c_{P}=\left(7.32 \mathrm{E}+4-9.05 \times T_{A v g}\right) / 42.4$ & $\mathrm{~J} /(\mathrm{kg} \mathrm{K})$ & {$[24]$} \\
$\begin{array}{l}\text { Species diffusion } \\
\text { coefficient }\end{array}$ & $D_{A B}=9.30 \mathrm{E}-8 \times \exp \left[-6.44 \mathrm{E}+3 /\left(1.99 \times T_{A v g}\right)\right]$ & $\mathrm{m}^{2} / \mathrm{s}$ & {$[25] \&[26]$} \\
\hline
\end{tabular}

The most common use of $\mathrm{Nu}$ and $\mathrm{Sh}$ correlations is to determine convection transfer coefficients $\left(h_{t h}\right.$ and $\left.h_{m}\right)$. However, in this study, the variable of interest is the purified crystal mass. This can be related to the length scale of both $\mathrm{Nu}$ and $\mathrm{Sh}$. The correlations, $\mathrm{Nu}$ or $S h=a(\operatorname{Gr} \operatorname{Pr} S c)^{b}$, can then be correlated to a dimensionless purified crystal mass ( $\left.\eta\right)$ [23] which is defined as

$$
\eta=\frac{m}{m_{0}}
$$

where $m$ is the purified crystal mass and $m_{0}$ is the initial $\mathrm{LiCl}$ mass in a $30 \mathrm{~g} \mathrm{LiCl}-\mathrm{CsCl}$ molten salt batch. The length scale and mass of the crystal are related with the purified crystal volume $(V)$ relationship to length scale $\left(L=V^{1 / 3}\right)$. The $V$ can be defined as the purified crystal mass divided by the purified crystal density $\left(\rho_{s}=2068 \mathrm{~kg} / \mathrm{m}^{3}\right.$ [15]) resulting in a length scale defined in terms of purified crystal mass; that is,

$$
L=\left(\frac{m}{\rho_{s}}\right)^{1 / 3}
$$

Thus, $\eta$ can be represented by

$$
\eta \propto N u^{3} \text { and } S h^{3}
$$


in terms of mass. With these proportionality relationships, it is reasonable to say that a typical form of a Nusselt or Sherwood correlation for natural convection can be used to describe the dimensionless purified crystal mass,

$$
\eta=a(\operatorname{Gr} \operatorname{Pr} S c)^{b}
$$

where the third power is being consumed into the constants $a$ and $b$. This correlation is only valid if constants $a$ and $b$ are greater than zero, because mass cannot be negative and zero mass is trivial.

The three crystal growth rate correlations were developed based on Eq. (3.13) to better understand crystal growth rate behavior and determine the rate controlling physics of the cold finger melt crystallization separating process. The first correlation assumes that purified crystal growth rate is controlled primarily by heat transfer whereas the second one assumes that purified crystal growth rate is controlled primarily by mass transfer. Finally, the third correlation takes into account both the heat and mass transfer effects on purified crystal growth rate. Fitted parameters for all three correlations are listed in Table 3.2. The $R^{2}$ values for all correlations seem to be on the same order and show no significant differences. However, by observing carefully, it can be seen that Eq. (3.13) provides the best fit indicating that both heat and mass transfers must be considered in scale-up processes. The results indicate that mass transfer has a more controlling effect on purified crystal growth rate than heat transfer. Also, by combining both effects, the exponent $(b)$ is closer to the mass transfer exponent than the heat transfer exponent.

These three correlations and their fitted lines to experimental data sets are compared in a log-log scale (see Figure 3.14). All three correlations are non-linear in nature, but by taking the log of the correlations they can be compared in a linear fashion. It is evident that the fits are similar between correlations with $R^{2}$ values having a maximum variance of 0.013 from each other and; indeed, the mass transfer component has the largest contribution to the magnitude of the independent variable of the correlation accounting for both heat and mass transfers. The evidence of this is shown by the closeness of the heat and mass transfer and mass transfer correlations' independent variable magnitudes (see Figure 3.14). 
Table 3.2: Three proposed dimensionless purified crystal mass correlations with respective $a, b$, correlation coefficient $(R)$, correlation of determination $\left(R^{2}\right)$, and probability function $\left(P_{C}\right)$ values discussed later.

\begin{tabular}{llccccc}
\hline Effects & Correlation & $a$ & $b$ & $R$ & $R^{2}$ & $P_{C}$ \\
\hline $\begin{array}{l}\text { Heat } \\
\text { Transfer }\end{array}$ & $\eta=a(G r P r)^{b}$ & $1.41 \mathrm{E}-10$ & 1.624 & 0.925 & 0.855 & $3.85 \mathrm{E}-13$ \\
$\begin{array}{l}\text { Mass } \\
\text { Transfer }\end{array}$ & $\eta=a(G r S c)^{b}$ & $1.26 \mathrm{E}-13$ & 1.586 & 0.930 & 0.864 & $1.64 \mathrm{E}-13$ \\
$\begin{array}{l}\text { Heat \& Mass } \\
\text { Transfers }\end{array}$ & $\eta=a(G r P r S c)^{b}$ & $2.52 \mathrm{E}-14$ & 1.565 & 0.932 & 0.868 & $1.05 \mathrm{E}-13$ \\
\hline
\end{tabular}

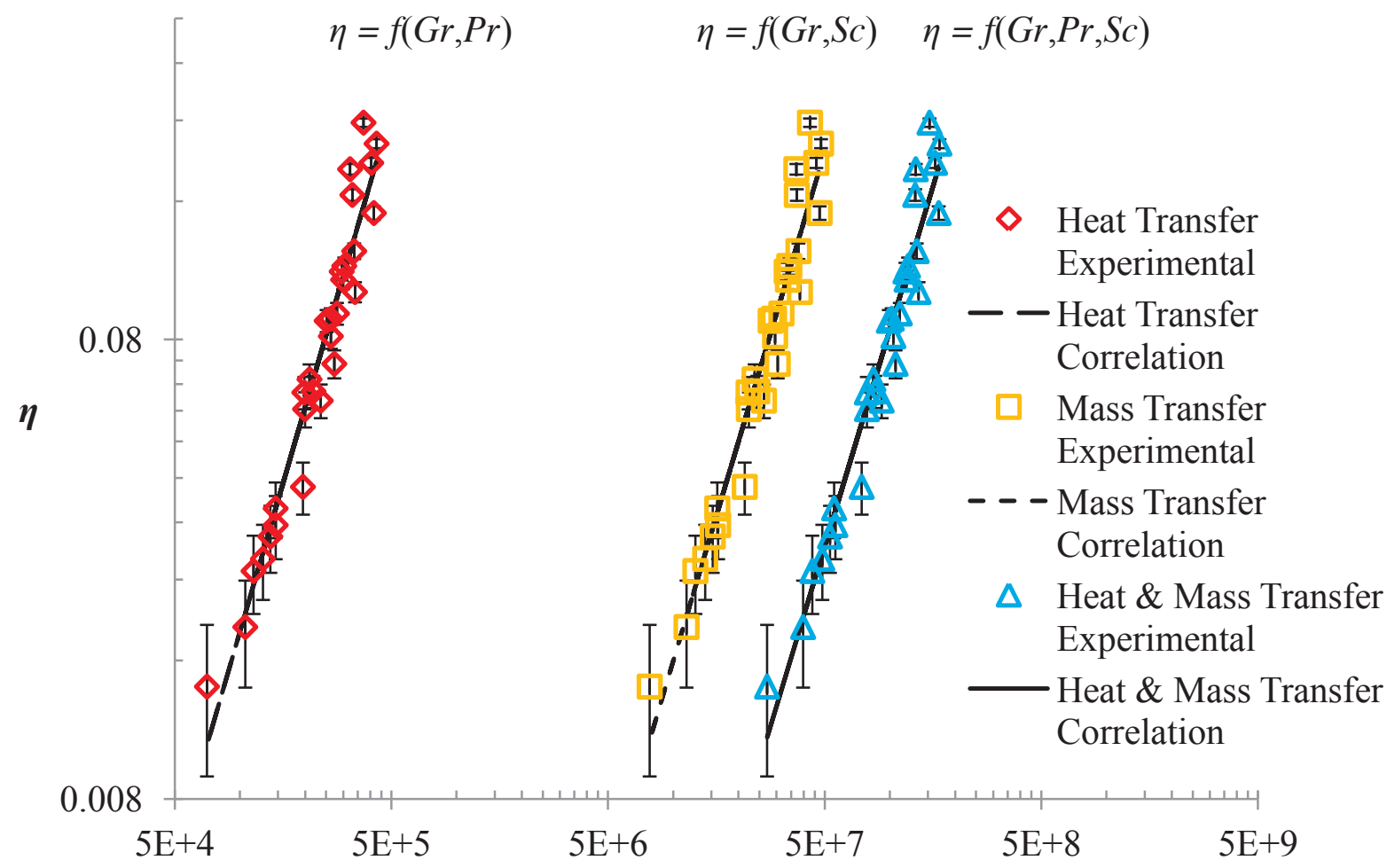

Dimensionless Parameter

Figure 3.14: Dimensionless crystal mass vs. respective dimensionless parameter including the effects of heat, mass, and heat \& mass transfers for experimental data and correlation predictions of experiments with varying initial compositions, crystal growth times, and cooling gas flow rates.

The correlation accounting for both heat and mass transfer effects was selected to represent all three correlations for the following discussion because it has the best fit to the experimental data of the three. The accuracy of the fit is shown in Figure 3.15, which compares the predicted $\eta$ from Eq. (3.13) to the measured $\eta$ from the experiments. Data that 
is above the line is under predicted by the correlation and the opposite is true for data underneath the line. It is readily apparent that the correlation fits the given data well at the slower cooling gas flow rate cases. Slightly scattered groups of data from the faster cooling gas flow rate cases can be observed upon careful inspection.

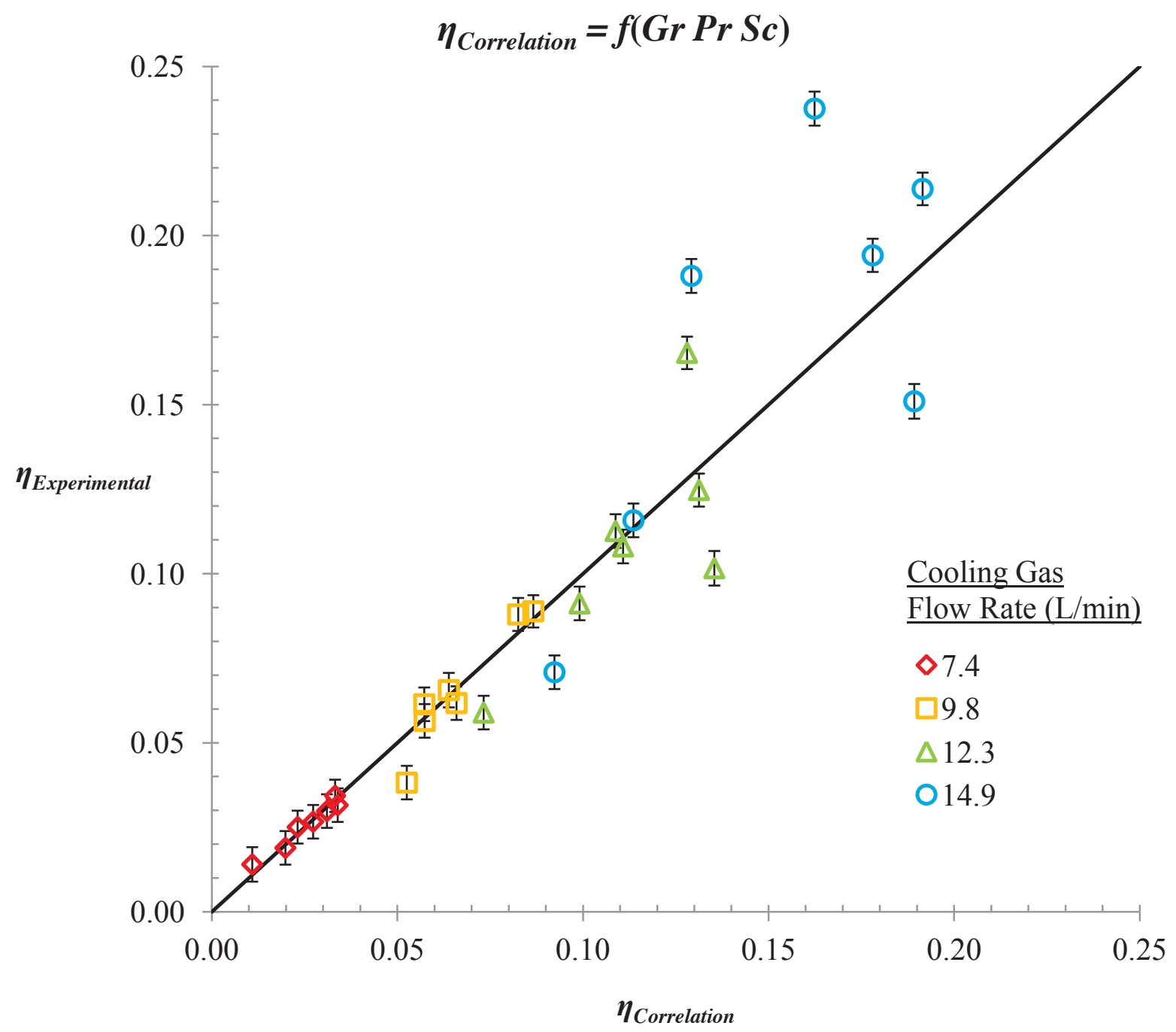

Figure 3.15: Experimental vs. correlation dimensionless crystal mass, where the correlation is a function of dimensionless $\mathrm{Gr}, \mathrm{Pr}$, and $S c$ for experiments with varying initial compositions, crystal growth times, and cooling gas flow rates.

The general comparison of the three correlations has provided useful insights as to how the correlations correlate to the data, it is also important to look at the statistical probability of correlation between the correlation predictions and the experimental data. Even though the three correlations are non-linear in nature, the correlation coefficient $(R)$ can 
be calculated based on a linear interpretation between experimental and predicted $\eta$. The expression of $R$ is given by:

$$
R=\frac{N \sum_{i}\left(\eta_{\text {exp }, i} \eta_{p, i}\right)-\left(\sum_{i} \eta_{\exp , i}\right)\left(\sum_{i} \eta_{p, i}\right)}{\sqrt{N \sum_{i} \eta_{\text {exp }, i}^{2}-\left(\sum_{i} \eta_{\text {exp }, i}\right)^{2}} \sqrt{N \sum_{i} \eta_{p, i}^{2}-\left(\sum_{i} \eta_{p, i}\right)^{2}}}
$$

where the subscripts exp,i and $p, i$ denote experimental and predicted values, respectively. To indicate the degree of correlation, a common test of the $R$ value with the probability distribution is derived from an uncorrelated parent population. In this case, a probability $P_{C}(R, N)$ test is utilized to determine the degree of correlation from $N$ uncorrelated experimental data points. The expression is given by [27]:

$$
P_{C}(R, N)=\frac{1}{\sqrt{\pi}} \frac{\Gamma\left(\frac{v+1}{2}\right)}{\Gamma\left(\frac{v}{2}\right)} \int_{|R|}^{1}\left(1-x^{2}\right)^{(v-2) / 2} d x
$$

where $\Gamma(k)$ is the Euler gamma function to the factorial function $k$ ! extended to non-integral arguments, and $v=N-2$. If the probability $P_{C}$ at the given $R$ and $N$ is less than 0.001 , then the probability is high that the experimental and predicted values are correlated and that the fit between them is justified. The values of $R, R^{2}$, and $P_{C}$ for different cooling gas flow rates of the system and the overall system were calculated for each correlation and are listed in Table 3.3 for comparison.

Of the three correlations for the overall system, it is apparent that the heat and mass transfer correlation has the highest $R$ value, with the lowest $P_{C}$ of $1.05 \mathrm{E}-13$ in comparison to the other correlations. It is important to, first, notice that high values of $R$ can be obtained by using a wide range of data, although the data may not be more accurate than for a narrow range [27]; and second, not to forget the underlying assumptions in deriving each correlation. Despite this consideration the data sets for the two fastest cooling gas flow rates have a relatively lower probability of correlation between experimental and predicted $\eta$ with $P_{C}>$ 0.001 (see Table 3.3). Therefore, to provide another meaningful comparison and to distinguish these models in statistical fashion, the residual plots $\left(\eta_{\text {exp }}-\eta_{p}\right)$ are shown in Figure 3.16 for all three correlations. The results indicate that all three correlations over and under predict experiments with the two fastest cooling gas flow rates for higher $\eta$ values. 
The analysis has shown that for slower cooling gas flow rates the correlations predict experimental $\eta$ well and that discretion should be used when predicting $\eta$ for faster cooling gas flow rates. The analysis has also shown that the correlation accounting for heat and mass transfer fits the experimental data best.

Table 3.3: The correlation coefficients $(R)$, coefficients of determination $\left(R^{2}\right)$, and probability functions $P_{C}(R, N)$ for all three correlations.

\begin{tabular}{|c|c|c|c|c|c|c|c|c|c|}
\hline \multirow{2}{*}{$\begin{array}{l}\text { Correlation } \\
\text { System } \\
\text { Description }\end{array}$} & \multicolumn{3}{|c|}{$\begin{array}{l}\text { Heat Transfer } \\
\eta=a(G r P r)^{b}\end{array}$} & \multicolumn{3}{|c|}{$\begin{array}{l}\text { Mass Transfer } \\
\eta=a(G r S c)^{b}\end{array}$} & \multicolumn{3}{|c|}{$\begin{array}{l}\text { Heat \& Mass Transfer } \\
\qquad \eta=a(G r \operatorname{Pr} S c)^{b}\end{array}$} \\
\hline & $R$ & $R^{2}$ & $P_{C}(R, N)$ & $R$ & $R^{2}$ & $P_{C}(R, N)$ & $R$ & $R^{2}$ & $P_{C}(R, N)$ \\
\hline \multicolumn{10}{|c|}{$\underline{\text { Cooling Gas Flow Rate }}$} \\
\hline $7.4 \mathrm{~L} / \mathrm{min}$ & 0.980 & 0.961 & $5.28 \mathrm{E}-05$ & 0.979 & 0.959 & 5.79E-05 & 0.979 & 0.958 & $6.22 \mathrm{E}-05$ \\
\hline $9.8 \mathrm{~L} / \mathrm{min}$ & 0.913 & 0.833 & $2.05 \mathrm{E}-03$ & 0.938 & 0.881 & $8.77 \mathrm{E}-04$ & 0.950 & 0.903 & $5.20 \mathrm{E}-04$ \\
\hline $12.3 \mathrm{~L} / \mathrm{min}$ & 0.756 & 0.571 & $2.47 \mathrm{E}-02$ & 0.754 & 0.568 & $2.52 \mathrm{E}-02$ & 0.752 & 0.566 & $2.55 \mathrm{E}-02$ \\
\hline $14.9 \mathrm{~L} / \mathrm{min}$ & 0.682 & 0.465 & $4.58 \mathrm{E}-02$ & 0.707 & 0.499 & $3.79 \mathrm{E}-02$ & 0.720 & 0.518 & $3.42 \mathrm{E}-02$ \\
\hline \multicolumn{10}{|l|}{ Overall } \\
\hline & 0.925 & 0.855 & $3.85 \mathrm{E}-13$ & 0.930 & 0.864 & $1.64 \mathrm{E}-13$ & 0.932 & 0.868 & $1.05 \mathrm{E}-13$ \\
\hline
\end{tabular}



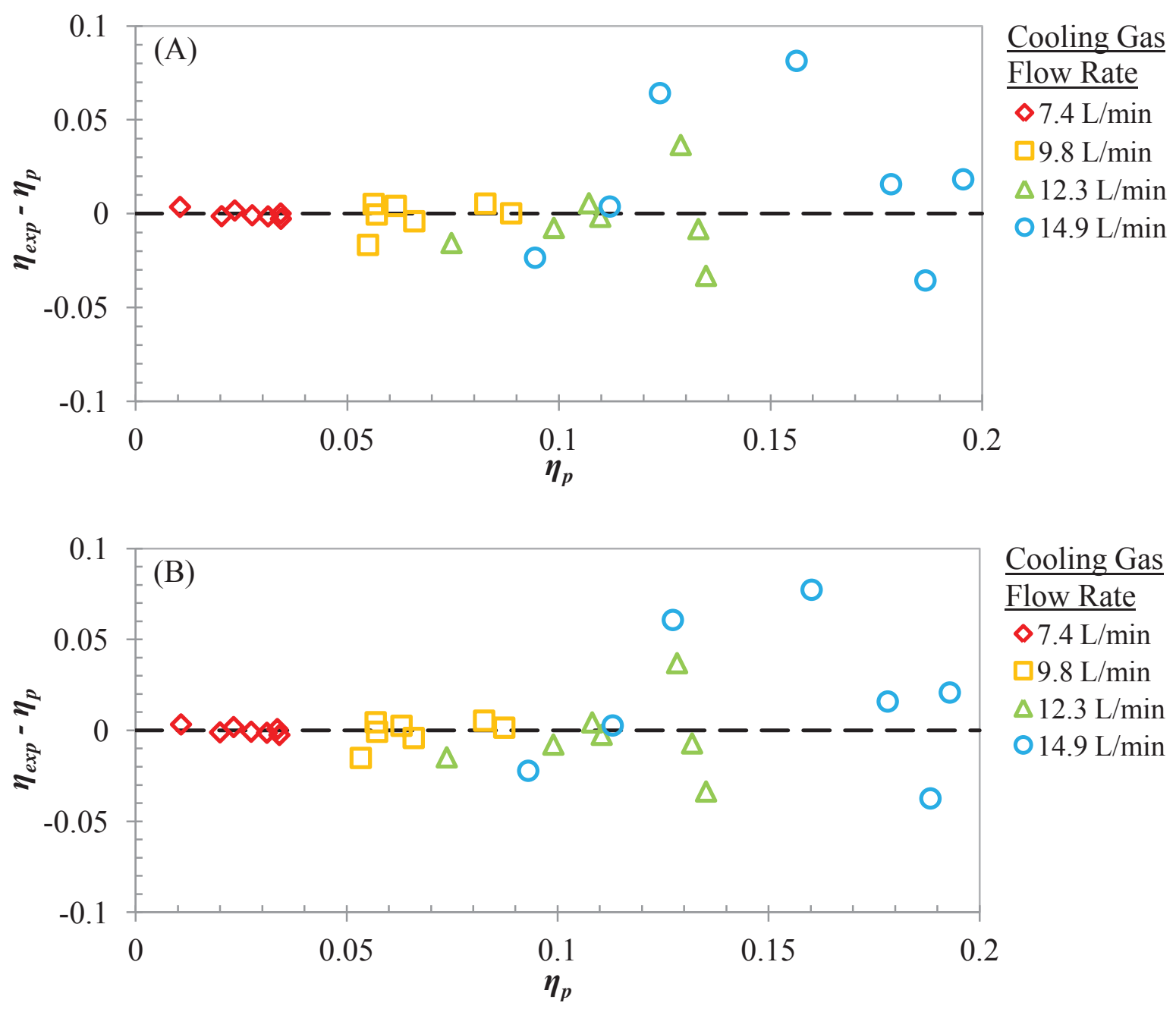

Cooling Gas

Flow Rate

$\diamond 7.4 \mathrm{~L} / \mathrm{min}$

$\square 9.8 \mathrm{~L} / \mathrm{min}$

$\triangle 12.3 \mathrm{~L} / \mathrm{min}$

$014.9 \mathrm{~L} / \mathrm{min}$

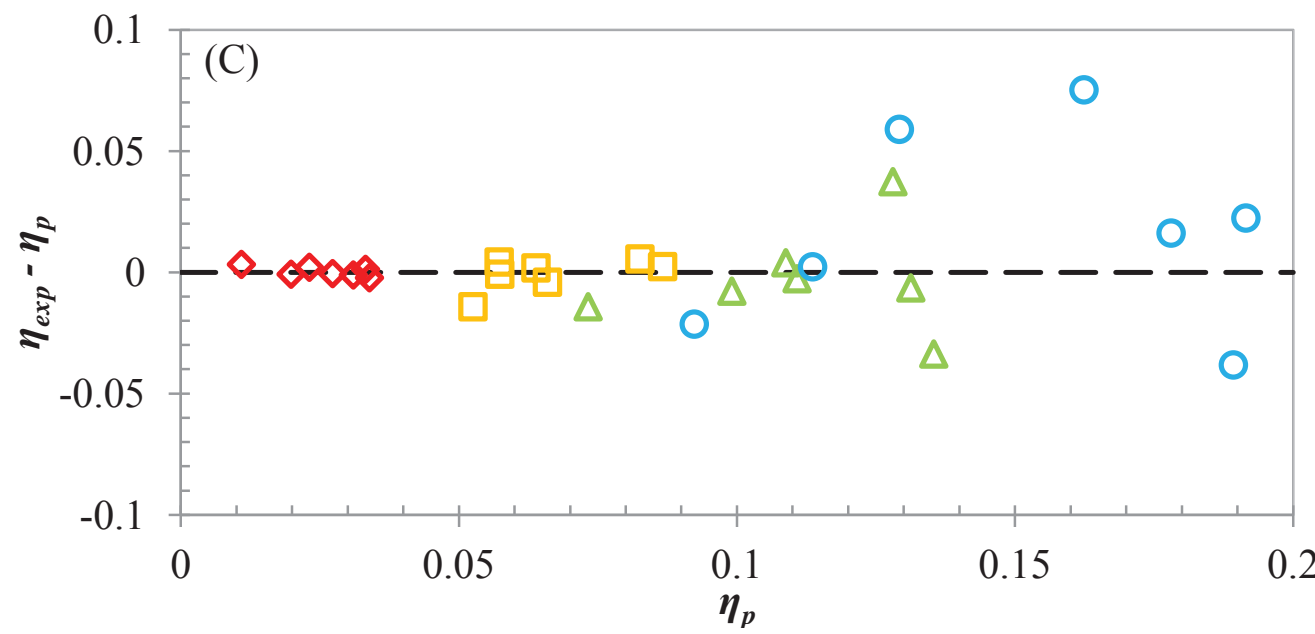

Cooling Gas

Flow Rate

$\diamond 7.4 \mathrm{~L} / \mathrm{min}$

$\square 9.8 \mathrm{~L} / \mathrm{min}$

$\triangle 12.3 \mathrm{~L} / \mathrm{min}$

$014.9 \mathrm{~L} / \mathrm{min}$

Figure 3.16: Residual vs. predicted $\boldsymbol{\eta}$ for: (A) heat transfer correlation, (B) mass transfer correlation, and $(\mathrm{C})$ heat and mass transfer correlation. 
With the given degrees of confidence, it would be interesting to determine the relative magnitudes of both heat and mass transfer coefficients. Here, estimated values of $h_{t h}$ and $h_{m}$ could be obtained as if $\eta=N u^{3}$ and $S h^{3}$. Although the proportionality in Eq. (3.12) does not imply equality, it can be used to obtain estimated values of $h_{t h}$ and $h_{m}$ as relative magnitudes. Knowing that $h_{t h}$ values for natural convective systems are reported to be within the range of $50-1000 \mathrm{~W} /\left(\mathrm{m}^{2} \mathrm{~K}\right)$ for liquids [23], the magnitude of the values were calculated on average as if Eq. (3.8) was an equality expression to see if calculated $h_{t h}$ averages for three correlations were within the reported range (see Table 3.4). The three correlations were for growth rate and the results show that the average relative $h_{t h}$ values are lower than the reported range. The average $h_{m}$ calculations for the correlations are also reported in Table 3.4 to show magnitudes of $10^{-7} \mathrm{~m} / \mathrm{s}$ for each correlation.

Table 3.4: Average $\boldsymbol{h}_{t h}$ and $\boldsymbol{h}_{m}$ for the three correlations.

\begin{tabular}{llccc}
\hline Effects & Correlation & Average $\eta$ & $\begin{array}{c}\text { Average } h_{t h} \\
\left(\mathrm{~W} / \mathrm{m}^{2} \mathrm{~K}\right)\end{array}$ & $\begin{array}{c}\text { Average } h_{m} \\
(\mathrm{~m} / \mathrm{s})\end{array}$ \\
\hline Heat Transfer & $\eta=a(G r P r)^{b}$ & $0.0893 \pm 0.0520$ & $26.2 \pm 1.64$ & $1.02 \mathrm{E}-07 \pm 7.03 \mathrm{E}-09$ \\
Mass Transfer & $\eta=a(G r S c)^{b}$ & $0.0889 \pm 0.0520$ & $26.1 \pm 1.57$ & $1.02 \mathrm{E}-07 \pm 6.72 \mathrm{E}-09$ \\
$\begin{array}{l}\text { Heat \& Mass } \\
\text { Transfers }\end{array}$ & $\eta=a(G r P r S c)^{b}$ & $0.0895 \pm 0.0524$ & $26.2 \pm 1.55$ & $1.02 \mathrm{E}-07 \pm 6.58 \mathrm{E}-09$ \\
\hline
\end{tabular}

It would now be interesting to know how the components of the independent variables of the three correlations behave throughout the least squares curve fitting process using the experiments performed at initial 1, 3, and $7.5 \mathrm{wt} \% \mathrm{CsCl}$ and determine the accuracy of its fitted correlations by predicting the experiments performed at initial $5 \mathrm{wt} \% \mathrm{CsCl}$. To investigate the independent variable components the three correlations for heat, mass, and heat \& mass transfers will now take the following form:

$$
\eta=a\left(G r^{b} \operatorname{Pr}^{c} S c^{d}\right)
$$

where $a, b, c$, and $d$ are constants that must be greater than zero for the correlations to be valid. The experimental date with initial 1, 3, and $7.5 \mathrm{wt} \% \mathrm{CsCl}$ was selected for curve fitting because of its distribution of purified crystal masses that provide values at the high, low, and mid ranges of all experiments performed. Once the fitted correlations were 
determined the accuracy of predicting the experiments with initial $5 \mathrm{wt} \% \mathrm{CsCl}$ was determined by calculating the root mean square deviation (RMSD) as a percent change from experimental data with the following expression:

$$
R M S D=100 \times \sqrt{\frac{\sum_{i=1}^{n}\left(\frac{\eta_{\text {exp }, i}-\eta_{p, i}}{\eta_{\text {exp }, i}}\right)^{2}}{n}}
$$

where $n$ is the number of experimental data points.

An iterative guess and check procedure was used with Microsoft Excel to minimize the RMSD of the correlations to the experimental data with initial 1, 3, and $7.5 \mathrm{wt} \% \mathrm{CsCl}$. The procedure began by guessing the previously calculated $a$ and $b$ for the new $a, b$, and $c$ for the correlation accounting for heat transfer. The new $a$ was manually adjusted and Excel's solver tool was used to minimize the RMSD by changing both $b$ and $c$ which were reset to the original guess before the next guess and check to ensure relative consistency of the solver. As $a$ was manually increased, the RMSD became smaller and the calculated exponents of $G r$ and $\operatorname{Pr}$ converged on the same value where the minimum RMSD was calculated (see Table 3.5). The convergence of the exponents $b$ and $c$ suggest that the previous heat transfer correlation is good and that it could take the form of $\eta=a R a^{b}$ where $R a$ is the Rayleigh number defined as the product of $\mathrm{Gr}$ and $\mathrm{Pr}$ and is a ratio of buoyancy forces to viscous forces [19]. Exponent $d$ was determined with the new correlation accounting for mass transfer and the exponent $b$ previously calculated with the new correlation accounting for heat transfer. The procedure for determining exponent $d$ began with a guess of the new coefficient $a$ and exponent $d$ as previously calculated in the first correlation accounting for mass transfer for all data. The new $a$ was manually adjusted and Excel's solver tool was used to minimize the RMSD by changing $d$ which was reset to the original guess before the next guess and check to ensure relative consistence of the solver. As $a$ was manually increased the RMSD became smaller until it was minimized and provided the best fit values for coefficient $a$ and exponent $d$ (see Table 3.5). With all the exponents determined the only coefficient left to solve was for the new correlation accounting for both heat and mass transfers. This coefficient was calculated with Excel's solver tool by changing the new $a$ to minimize the RMSD (see Table 3.5). 
Table 3.5: Three new proposed dimensionless purified crystal mass correlations with respective $a, b, c, d$, $R^{2}$, and $R M S D$ values for experiments performed with initial 1,3 , and $7.5 \mathrm{wt} \% \mathrm{CsCl}$.

\begin{tabular}{llccccc}
\hline Effects & Correlation & $a$ & $b \& c$ & $d$ & $R^{2}$ & $R M S D$ \\
\hline $\begin{array}{l}\text { Heat } \\
\text { Transfer }\end{array}$ & $\eta=a\left(G r^{b} \operatorname{Pr}^{c}\right)$ & $1.07 \mathrm{E}-09$ & 1.460 & 0.524 & 0.908 & 13.69 \\
$\begin{array}{l}\text { Mass } \\
\text { Transfer }\end{array}$ & $\eta=a\left(G r^{b} S c^{d}\right)$ & $2.94 \mathrm{E}-10$ & 1.460 & 0.524 & 0.908 & 13.69 \\
$\begin{array}{l}\text { Heat \& Mass } \\
\text { Transfers }\end{array}$ & $\eta=a\left(G r^{b} \operatorname{Pr}^{c} S c^{d}\right)$ & $4.67 \mathrm{E}-11$ & 1.460 & 0.524 & 0.905 & 13.74 \\
\hline
\end{tabular}

An analysis of the $R^{2}$ values for the new correlations and a comparison of the overall $R^{2}$ values for both new and first correlations provide additional insight to the crystal growth behavior. $R^{2}$ values were calculated for the new correlation fits first for experiments performed with $7.4 \mathrm{~L} / \mathrm{min}$ cooling gas flow rate, then for both 7.4 and $9.8 \mathrm{~L} / \mathrm{min}$ cooling gas flow rate, and so on until all cooling gas flow rates were included. The data sets show how uniform and predictable purified crystal mass data is for lower cooling gas flow rates and how it becomes more scattered and less predictable for higher cooling gas flow rates (see Table 3.6). The $R^{2}$ values for the three new correlations are higher than for the first three correlations and suggest that the purified crystal mass for experiments preformed at an initial $5 \mathrm{wt} \% \mathrm{CsCl}$ are (1) slightly shifted from that of the other experiments or (2) more scattered than the other experiments. A potential reason for these suggestions is the addition of more than just 15 min crystal growth times that could potentially shift or scatter this data. A plot of $\eta$ versus $G r$ provide a visual representation of the fitted correlations to the experimental data performed with initial 1, 3, and $7.5 \mathrm{wt} \% \mathrm{CsCl}$ (see Figure 3.17).

Table 3.6: $R^{2}$ values for new correlations fitted to experiments performed with initial 1,3 , and $7.5 \mathrm{wt} \%$ $\mathrm{CsCl}$ for various combinations progressively including more cooling gas flow rate data sets.

\begin{tabular}{|c|c|c|c|c|c|}
\hline Effects & Correlation & $7.4 \mathrm{~L} / \mathrm{min}$ & + 9.8 L/min & $+12.3 \mathrm{~L} / \mathrm{min}$ & Overall \\
\hline $\begin{array}{l}\text { Heat } \\
\text { Transfer }\end{array}$ & $\eta=a\left(G r^{b} \operatorname{Pr}^{c}\right)$ & 1.00 & 0.994 & 0.873 & 0.908 \\
\hline $\begin{array}{l}\text { Mass } \\
\text { Transfer }\end{array}$ & $\eta=a\left(G r^{b} S c^{d}\right)$ & 1.00 & 0.994 & 0.873 & 0.908 \\
\hline $\begin{array}{l}\text { Heat \& Mass } \\
\text { Transfers }\end{array}$ & $\eta=a\left(G r^{b} \operatorname{Pr}^{c} S c^{d}\right)$ & 1.00 & 0.996 & 0.872 & 0.905 \\
\hline
\end{tabular}



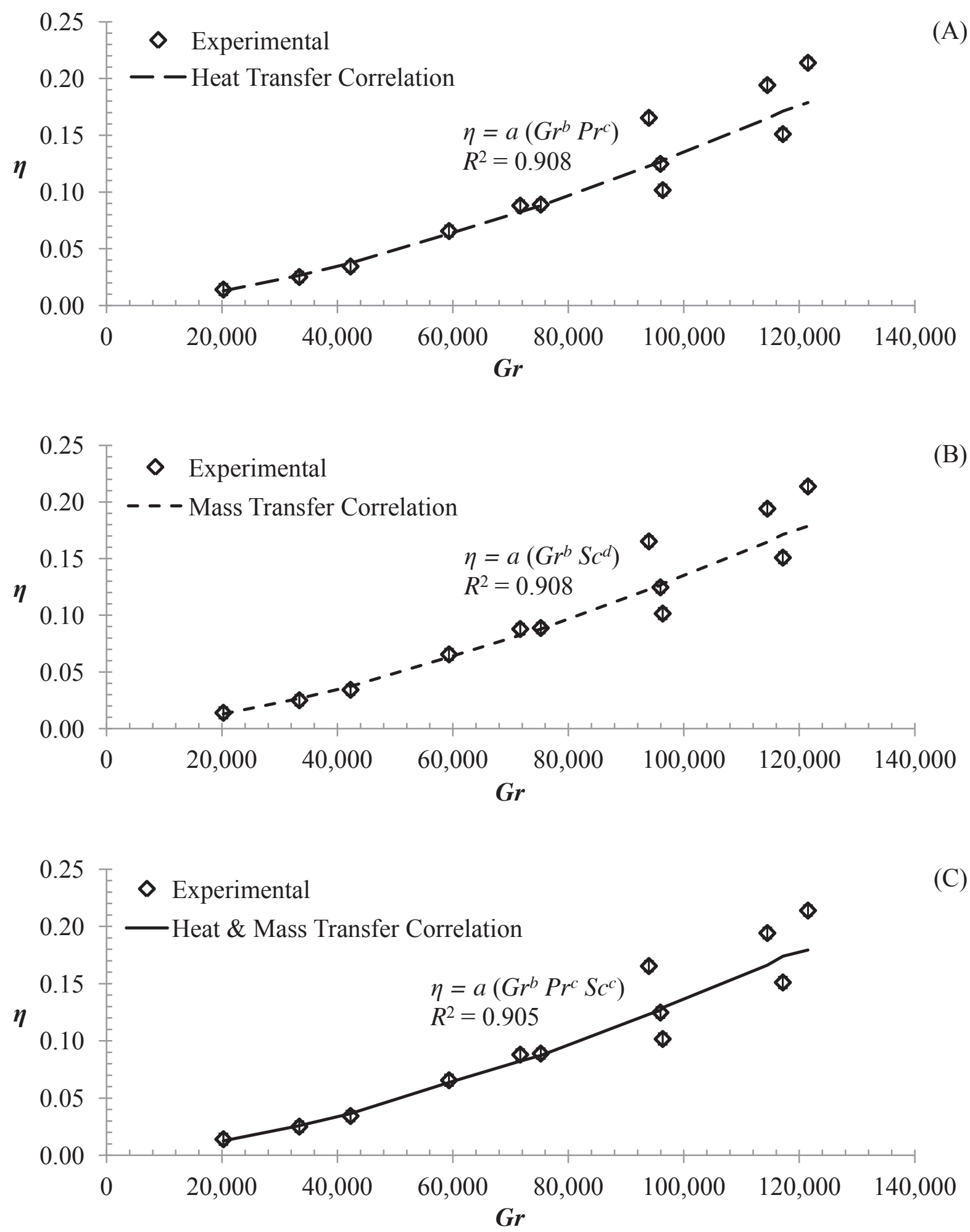

Figure 3.17: $\eta$ vs. Gr for new: (A) heat transfer, (B) mass transfer, and (C) heat and mass transfer correlations fitted to experimental data performed with initial 1,3 , and $7.5 \mathrm{wt} \% \mathrm{CsCl}$. 
The three new correlations obtained from curve fitting experiments performed with initial 1, 3, and $7.5 \mathrm{wt} \% \mathrm{CsCl}$ were used to predict the data obtained from experiments performed with initial $5 \mathrm{wt} \% \mathrm{CsCl}$. The accuracy of the prediction to the experiments was determined as mentioned before with the RMSD. The RMSD has approximately a $60 \%$ increase for the prediction of the experiments performed with initial $5 \mathrm{wt} \% \mathrm{CsCl}$ compared to the other experimental data with initial 1, 3, and $7.5 \mathrm{wt} \% \mathrm{CsCl}$ (see Table 3.7). A plot of $\eta$ versus $G r$ visually shows that most of the experimental data performed with initial $5 \mathrm{wt} \%$ $\mathrm{CsCl}$ is over predicted by the new correlations (see Figure 3.18). The plot shows 2 data points that are less accurately predicted than the others for the $14.9 \mathrm{~L} / \mathrm{min}$ cooling gas flow rate. These two data points are for crystal growth times of 15 and $30 \mathrm{~min}$ and are most likely due to human error in experiments. It would be expected that $\operatorname{Exp} 4(30,14.9)$ would be relatively larger than other crystals because of the extended crystal growth time compared to other experiments. The plot also confirms previous observations that cooling gas flow rate has the greatest effect on the crystal growth rate compared to initial $\mathrm{CsCl}$ concentration and crystal growth time, indicated by the segregated grouping for each cooling gas flow rate along the three correlation profiles; that is, data becomes more scattered for higher cooling gas flow rates.

Table 3.7: Three new proposed dimensionless purified crystal mass correlations with respective $a, b, c, d$, $R^{2}$, and $R M S D$ values of predicting experiments performed with initial $5 \mathrm{wt} \% \mathrm{CsCl}$.

\begin{tabular}{llccccc}
\hline Effects & Correlation & $a$ & $b \& c$ & $d$ & $R^{2}$ & $R M S D$ \\
\hline $\begin{array}{l}\text { Heat } \\
\text { Transfer }\end{array}$ & $\eta=a\left(G r^{b} \operatorname{Pr}^{c}\right)$ & $1.07 \mathrm{E}-09$ & 1.460 & 0.524 & 0.844 & 22.5 \\
$\begin{array}{l}\text { Mass } \\
\text { Transfer }\end{array}$ & $\eta=a\left(G r^{b} S c^{d}\right)$ & $2.94 \mathrm{E}-10$ & 1.460 & 0.524 & 0.844 & 22.5 \\
$\begin{array}{l}\text { Heat \& Mass } \\
\text { Transfers }\end{array}$ & $\eta=a\left(G r^{b} \operatorname{Pr}^{c} S c^{d}\right)$ & $4.67 \mathrm{E}-11$ & 1.460 & 0.524 & 0.856 & 21.4 \\
\hline
\end{tabular}




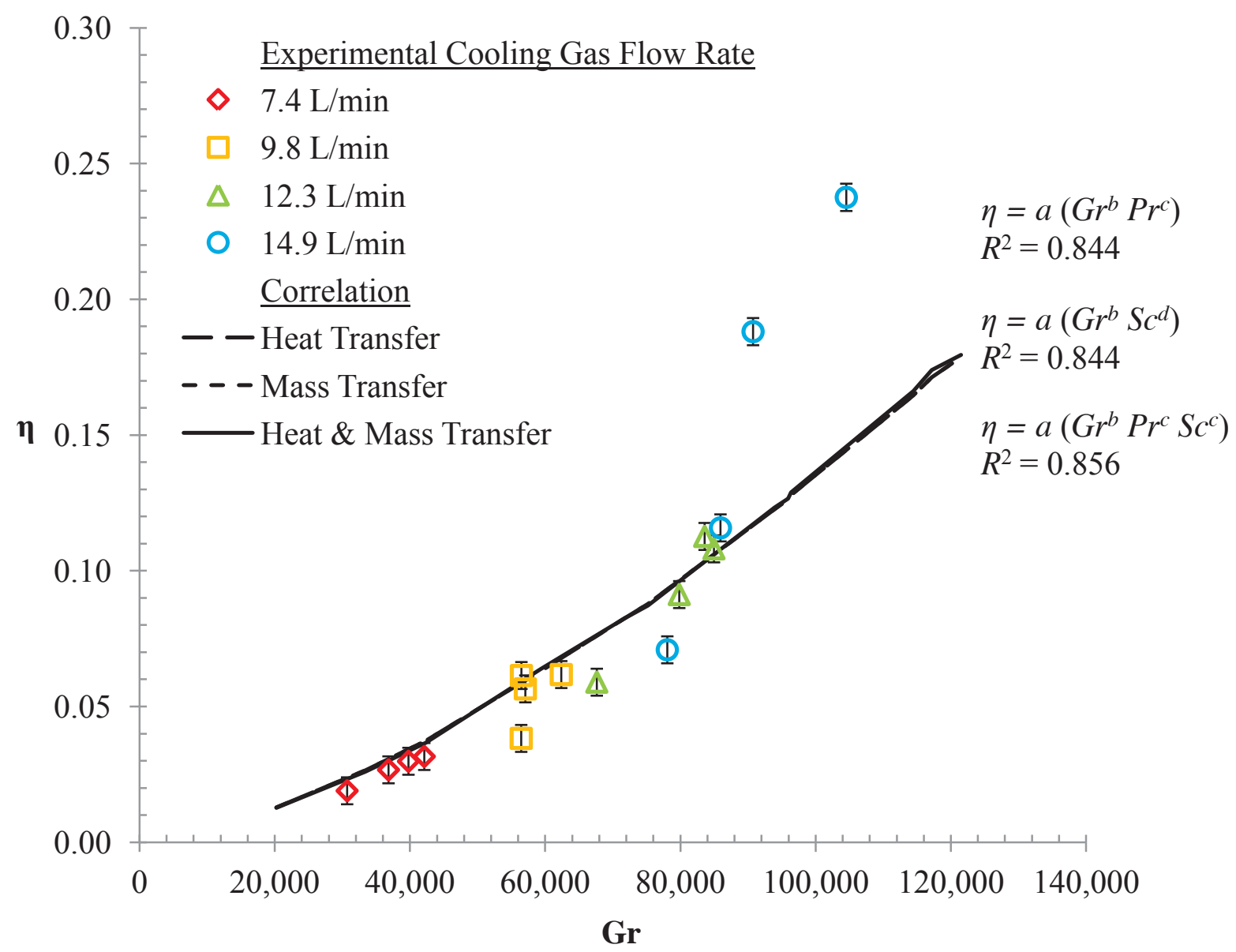

Figure 3.18: $\eta$ vs. $G r$ for new correlations predicting experimental data performed with initial 5 wt $\%$ CsCl.

In summary, the overall correlation and experimental analyses has provided two major discoveries. The first significant discovery was the effect of a $\mathrm{CsCl}$ concentrated drip formation that decreases crystal purity, especially for smaller crystal formations. There are, however, simple engineering controls that can reduce the negative effects of the concentrated drip, such as air jets that blow the drip away from the crystal, rapid movements of the cold finger that shake the drip away from the crystal, and wiping processes. The second significant discovery of the analyses performed showed that cooling gas flow rate most strongly controlled the production rate of the separation process compared to the other operating parameters of initial $\mathrm{CsCl}$ concentration and crystal growth time. 


\section{Chapter 4 Conclusions and Recommendations}

\subsection{Summary}

Cold finger melt crystallization separation experiments under different parametric effects were proposed and conducted to further develop pyrochemical technology. The experimental $\mathrm{LiCl}-\mathrm{CsCl}$ operating temperature was $650^{\circ} \mathrm{C}$ with a cold finger temperature held at the freezing temperature of $\mathrm{LiCl}\left(\approx 605^{\circ} \mathrm{C}\right)$. Molten salt and cold finger exhaust temperatures were measured throughout experiments and argon cooling gas flow was controlled manually. CsCl concentration was measured via ICP-MS for purified LiCl crystals and concentrated $\mathrm{LiCl}-\mathrm{CsCl}$ bulk. The varied parameters of interest were initial $\mathrm{CsCl}$ concentration, crystal growth time, and cooling gas flow rate because they all directly affect the production and purity of purified electrolyte.

Analysis of experiments with initial $5 \mathrm{wt} \% \mathrm{CsCl}$ concentration showed that for an experimental matrix of varying cold finger cooling gas flow rates $(7.4,9.8,12.3$, and 14.9 $\mathrm{L} / \mathrm{min})$ and crystal growth times $(5,10,15$, and $30 \mathrm{~min})$, the optimal cooling gas flow rate and crystal growth time of this experimental setup was $14.9 \mathrm{~L} / \mathrm{min}$ and $15 \mathrm{~min}$, respectively, producing a $0.33 \mathrm{wt} \% \mathrm{CsCl}$ crystal purity at a production rate of $0.36 \mathrm{~g} / \mathrm{min}$. Experimental results for initial concentrations of 1,3 , and $7.5 \mathrm{wt} \% \mathrm{CsCl}$ at the aforementioned coolant flow rates for separation times of $15 \mathrm{~min}$ were analyzed and showed that as initial $\mathrm{CsCl}$ concentration increased from 1 to $7.5 \mathrm{wt} \% \mathrm{CsCl}$, the change in purified crystal concentration for experiments was less than $0.5 \mathrm{wt} \% \mathrm{CsCl}$ for all but one data point. The optimal result of the initial $5 \mathrm{wt} \% \mathrm{CsCl}$ experiments along with results of initial 1, 3, and $7.5 \mathrm{wt} \% \mathrm{CsCl}$ bulk concentration experiments were used to predict a scale-up scenario. The scenario was to concentrate a $700 \mathrm{~g}$ batch of $\mathrm{LiCl}-\mathrm{CsCl}$ salt from 5 to $7 \mathrm{wt} \% \mathrm{CsCl}$ requiring 7 crystal growth cycles with 7 cold fingers. This scale-up scenario provided a potential separation rate and purity of $136 \mathrm{~g} / \mathrm{hr}$ and $0.24 \mathrm{wt} \% \mathrm{CsCl}$, respectively. This provides insight that will aid in future optimization and scale-up of cold finger separation, as well as economic feasibility as a commercial separation technique.

Six crystal growth rate correlations were developed to better understand crystal growth rate behavior and determine the effects of heat and mass transfers on the cold finger 
melt crystallization separation process. The correlation accounting for both heat and mass transfer in the molten salt system fits well to all the experimental data with an $R^{2}$ value of 0.87 and $P_{C}<0.001$. The result indicates that mass transfer had a slightly more dominant effect on purified crystal growth rate than heat transfer. Analysis of the first three correlations also showed that they predict experimental data well for slower cooling gas flow rates and not as well for faster cooling gas flow rates because operation at slower cooling gas flow rates is closer to thermodynamic equilibrium then operation at faster cooling gas flow rates. Relative values of heat and mass transfer coefficients were estimated based on the degree of accuracy for these correlations. Further studies would be necessary in order to obtain the actual values of these coefficients through dimensional analysis. Three additional correlations were proposed to study the behavior of the components of the independent variables of the first three correlations using the experimental data performed with initial 1, 3 , and $7.5 \mathrm{wt} \% \mathrm{CsCl}$, and the new correlations were used to predict the rest of the data (experiments performed with initial $5 \mathrm{wt} \% \mathrm{CsCl}$ ) not used to curve fit the new correlations.

The three new correlations provided predictions of the experimental data performed at initial $5 \mathrm{wt} \% \mathrm{CsCl}$ with average percent change root mean square deviation $(R M S D)$ of 22.1 and $R^{2}$ of 0.848 .

The overall analyses of this work has revealed two major discoveries of cold finger melt crystallization as a viable means of purifying used electrolytic oxide reduction electrolyte. The first significant discovery was the effect of a $\mathrm{CsCl}$ concentrated drip formation on purified crystal that decreases crystal purity, especially for smaller crystal formations where drip mass fractions are larger. The second significant discovery shown by the analyses was that cooling gas flow rate controls the production rate of cold finger separation more than both initial $\mathrm{CsCl}$ concentration and crystal growth time.

\subsection{Recommendations for Future Work}

This work has provided a fundamental study of cold finger melt crystallization as a viable means of purifying used oxide reduction electrolyte with a simplified LiCl-CsCl salt system. The next step for this work would be to perform experiments for the scale-up scenario to validate the production and purity predictions. The experiments should include the same salt system used in this work and other more complex salt systems to see how 
additional fission products effect purified electrolyte production and purity. Experiments performed with used electrolyte that was used to process commercial oxide fuel in the bench scale oxide reduction setup at the INL would be beneficial in understanding used electrolyte from the electrolytic oxide reduction process. Future work for literature review would be to benchmark cold finger separation production and purity against all other separation techniques and determine its economic feasibility. The other separations should at least include zone freezing and draw down techniques. 


\section{Bibliography}

[1] A. Andrews, "Nuclear Fuel Reprocessing: U.S. Policy Development," Congressional Research Service, Order Code RS22542, 2008.

[2] J. K. Shultis and R. E. Faw, Fundamentals of nuclear Science and Engineering, Boca Raton: CRC Press, 2008.

[3] "U.S. Department of Energy," [Online]. Available: http://www.ne.doe.gov/fuelcycle/neFuelCycle.html. [Accessed 8 January 2013].

[4] M. F. Simpson and J. D. Law, "Nuclear Fuel Reprocessing," Idaho National Laboratory, INL/EXT-10-17753, 2010.

[5] S. D. Herrmann, S. X. Li, M. F. Simpson and S. Phongikaroon, "Electrolytic Reduction of Spent Nuclear Oxide Fuel as Part of an Integral Process to Separate and Recover Actinides from Fission Procucts," Separation Science and Technology, vol. 41, pp. 1965-1983, 2006.

[6] Y. Cho, G. Park, H. Lee, I. Kim and D. Han, "Concentration of Cesium and Strontium Elements Involved in a LiCl Waste Salt by a Melt Crystallization Process," Nuclear Technology, vol. 171, pp. 325-334, 2010.

[7] B. H. Park, I. W. Lee and C. -S. Seo, "Electrolytic reduction behavior of U3O8 in a molten LiCl-Li2O salt," Chemical Engineering Science, vol. 63, pp. 3485-3492, 2008.

[8] L. J. Koch, Experimental Breeder Reactor II: An Integrated Experimental Fast Reactor Nuclear Power Station, La Grange Park, IL: American Nuclear Society, 2008.

[9] K. M. Goff, R. W. Benedict and D. Levniskas, "Spent Fuel Treatment at ANL-West," in DOE Spent Nuclear Fuel Challenges and Initiatives, Salt Lake City, UT, 1994.

[10] J. L. Willit, W. E. Miller and J. E. Battles, "Electrorefining of uranium and plutonium A literature review," Journal of Nuclear Materials, vol. 195, pp. 229-249, 1992. 
[11] J. J. Laidler, J. E. Battles, W. E. Miller, J. P. Ackerman and E. L. Carls, "Development of Pyroprocessing Technology," Progress in Nuclear Energy, vol. 31, pp. 131-140, 1997.

[12] R. Benedict, C. Solbrig, B. Westphal, T. A. Johnson, S. X. Li, K. Marsden and K. M. Goff, "Pyroprocessing Progress at Idaho National Laboratory," in Global 2007, Boise, ID, 2007.

[13] S. X. Li, T. A. Johnson, B. R. Westphal, K. M. Goff and R. W. Benedict, "Electrorefining Experience For Pyrochemical Processing Of Spent EBR-II Driver Fuel," in Global 2005, Tsukuba, Japan, 2005.

[14] A. N. Williams, S. Phongikaroon and M. F. Simpson, "Separation of CsCl from a ternary $\mathrm{CsCl}-\mathrm{LiCl}-\mathrm{KCl}$ salt via a melt crystallization technique for pyroprocessing waste minimization," Chemical Engineering Science, vol. 89, pp. 258-263, 2013.

[15] R. C. Weast, Handbook of Chemistry and Physics, Cleveland: CRC Press, Inc., 1976, pp. B-125.

[16] "FactSage," [Online]. Available: http://www.factsage.cn/fact/documentation/FTsalt/CsCl-LiCl.jpg. [Accessed 21 January 2013].

[17] G. J. Janz, "Thermodynamic and Transport Properties for Molten Salts: Correlation Equations for Critically Evaluated Density, Surface Tension, Electrical Conductance, and Viscosity Data," Journal of Physical and Chemical Reference Data, vol. 17, no. 2, pp. 63-289, 1988.

[18] R. B. Bird, W. E. Stewart and E. N. Lightfoot, Transport Phenomena, New York, NY: John Wiley \& Sons, Inc., 2007, pp. 513-542.

[19] Y. A. Cengel and J. M. Cimbala, Fluid Mechanics Fundamentals and Applications 2nd Edition, New York, NY: McGraw-Hill Companies, Inc., 2010, pp. 283-319. 
[20] V. A. Khokhlov, A. O. Kodintseva and E. S. Z. Filatov, ""Anomalous" Thermal Conductivity of Crystalline Alkali Halides Close to Their Melting Point," Naturforsch., A: Astrophys., Phys. Chem., vol. 48A, pp. 595-598, 1993.

[21] Y. Nagasaka, N. Nakazawa and A. Nagashima, "Experimental determination of the thermal diffusivity of molten alkali halides by the forced Rayleigh Scattering method. I. molten $\mathrm{LiCl}, \mathrm{NaCl}, \mathrm{KCl}, \mathrm{RbCl}$, and $\mathrm{CsCl}$," International Journal of Thermophysics, vol. 13, pp. 555-574, 1992.

[22] D. W. Green and R. H. Perry, Perry's Chemical Engineers' Handbook, 8th ed., New York, NY: McGraw-Hill, 2008, pp. 2-146, 2-159.

[23] F. P. Incropera, D. P. Dewitt, T. L. Bergman and A. S. Lavine, Fundamentals of Heat and Mass Transfer 6th Edition, Hoboken, NJ: John Wiley \& Sons, Inc., 2007, pp. 8, 259-263, 374-381, 559-597.

[24] NIST, "National Institute of Standards and Technology," NIST, [Online]. Available: http://webbook.nist.gov/cgi/cbook.cgi? ID=C7447418\&Type=JANAFL\&Table=on . [Accessed 2 July 2013].

[25] G. J. Janz and N. P. Bansal, "Molten Salts Data: Diffusion Coefficients in Single and Multi-Component Salt Systems," J. Phys. Chem. Ref. Data, vol. 11, no. 3, pp. 522-523, 1982.

[26] J. M. Prausnitz, R. N. Lichtenthaler and E. Gomes de Azevedo, Molecular Thermodynamics of Fluid-Phase Equilibria, 3rd ed., Upper Saddle River, NJ: PrenticeHall, Inc., 1999, p. 844.

[27] P. R. Bevingtion, Data Reduction and Error Analysis for the Physical Sciences, New York, NY: McGraw-Hill Book Company, 1969, pp. 122-129. 


\section{Appendices}

\section{Appendix A - Experimental Mass Data}

\begin{tabular}{|c|c|c|c|c|c|c|}
\hline Experiment & $\begin{array}{l}\text { Cold Finger } \\
\text { Mass (g) }\end{array}$ & \pm & $\begin{array}{l}\text { Cold Finger + } \\
\text { Crystal + Drip } \\
\text { Mass (g) }\end{array}$ & \pm & $\begin{array}{l}\text { Crystal + Drip } \\
\text { Mass }(\mathrm{g})\end{array}$ & \pm \\
\hline $\operatorname{Exp} 1 \mathrm{a}(1,11.0)$ & 190.05 & 0.1 & 190.64 & 0.1 & 0.59 & 0.14 \\
\hline $\operatorname{Exp} 1 \mathrm{a}(2,11.0)$ & 190.05 & 0.1 & 190.94 & 0.1 & 0.89 & 0.14 \\
\hline $\operatorname{Exp} 1 \mathrm{a}(3,11.0)$ & 190.05 & 0.1 & 191.39 & 0.1 & 1.34 & 0.14 \\
\hline $\operatorname{Exp} 1 \mathrm{a}(4,11.0)$ & 190.05 & 0.1 & 191.74 & 0.1 & 1.69 & 0.14 \\
\hline $\operatorname{Exp} 1 \mathrm{a}(5,11.0)$ & 190.05 & 0.1 & 191.88 & 0.1 & 1.83 & 0.14 \\
\hline $\operatorname{Exp} 1 \mathrm{a}(10,11.0)$ & 190.05 & 0.1 & 193.20 & 0.1 & 3.15 & 0.14 \\
\hline $\operatorname{Exp} 1 \mathrm{a}(15,11.0)$ & 190.05 & 0.1 & 194.15 & 0.1 & 4.10 & 0.14 \\
\hline $\operatorname{Exp} 1 \mathrm{a}(30,11.0)$ & 190.05 & 0.1 & 195.27 & 0.1 & 5.22 & 0.14 \\
\hline $\operatorname{Exp} 1 \mathrm{a}(60,11.0)$ & 190.05 & 0.1 & 198.92 & 0.1 & 8.87 & 0.14 \\
\hline $\operatorname{Exp} 1 b(30,5.1)$ & 190.05 & 0.1 & 190.24 & 0.1 & 0.19 & 0.14 \\
\hline $\operatorname{Exp} 1 b(30,6.2)$ & 190.05 & 0.1 & 190.44 & 0.1 & 0.39 & 0.14 \\
\hline $\operatorname{Exp} 1 b(30,6.8)$ & 190.05 & 0.1 & 190.64 & 0.1 & 0.59 & 0.14 \\
\hline $\operatorname{Exp} 1 b(30,7.4)$ & 190.05 & 0.1 & 191.34 & 0.1 & 1.29 & 0.14 \\
\hline $\operatorname{Exp} 1 b(30,8.5)$ & 190.05 & 0.1 & 191.81 & 0.1 & 1.76 & 0.14 \\
\hline $\operatorname{Exp} 1 b(30,9.8)$ & 190.05 & 0.1 & 193.67 & 0.1 & 3.62 & 0.14 \\
\hline $\operatorname{Exp} 1 b(30,11.0)$ & 190.05 & 0.1 & 194.67 & 0.1 & 4.62 & 0.14 \\
\hline $\operatorname{Exp} 2(15,7.4)$ & 190.08 & 0.1 & 191.10 & 0.1 & 1.02 & 0.14 \\
\hline $\operatorname{Exp} 2(15,9.8)$ & 190.08 & 0.1 & 192.72 & 0.1 & 2.64 & 0.14 \\
\hline $\operatorname{Exp} 2(15,12.3)$ & 190.08 & 0.1 & 194.99 & 0.1 & 4.91 & 0.14 \\
\hline $\operatorname{Exp} 2(15,14.9)$ & 190.08 & 0.1 & 196.43 & 0.1 & 6.35 & 0.14 \\
\hline $\operatorname{Exp} 3(15,7.4)$ & 190.08 & 0.1 & 190.81 & 0.1 & 0.73 & 0.14 \\
\hline
\end{tabular}




\begin{tabular}{|c|c|c|c|c|c|c|}
\hline $\operatorname{Exp} 3(15,9.8)$ & 190.08 & 0.1 & 192.64 & 0.1 & 2.56 & 0.14 \\
\hline $\operatorname{Exp} 3(15,12.3)$ & 190.08 & 0.1 & 193.71 & 0.1 & 3.63 & 0.14 \\
\hline $\operatorname{Exp} 3(15,14.9)$ & 190.08 & 0.1 & 195.73 & 0.1 & 5.65 & 0.14 \\
\hline $\operatorname{Exp} 4(5,7.4)$ & 190.06 & 0.1 & 190.60 & 0.1 & 0.54 & 0.14 \\
\hline $\operatorname{Exp} 4(5,9.8)$ & 190.06 & 0.1 & 191.15 & 0.1 & 1.09 & 0.14 \\
\hline $\operatorname{Exp} 4(5,12.3)$ & 190.06 & 0.1 & 191.74 & 0.1 & 1.68 & 0.14 \\
\hline $\operatorname{Exp} 4(5,14.9)$ & 190.06 & 0.1 & 192.08 & 0.1 & 2.02 & 0.14 \\
\hline $\operatorname{Exp} 4(10,7.4)$ & 190.06 & 0.1 & 190.82 & 0.1 & 0.76 & 0.14 \\
\hline $\operatorname{Exp} 4(10,9.8)$ & 190.06 & 0.1 & 191.82 & 0.1 & 1.76 & 0.14 \\
\hline $\operatorname{Exp} 4(10,12.3)$ & 190.06 & 0.1 & 192.66 & 0.1 & 2.60 & 0.14 \\
\hline $\operatorname{Exp} 4(10,14.9)$ & 190.06 & 0.1 & 193.36 & 0.1 & 3.30 & 0.14 \\
\hline $\operatorname{Exp} 4(15,7.4)$ & 190.06 & 0.1 & 190.91 & 0.1 & 0.85 & 0.14 \\
\hline $\operatorname{Exp} 4(15,9.8)$ & 190.06 & 0.1 & 191.81 & 0.1 & 1.75 & 0.14 \\
\hline $\operatorname{Exp} 4(15,12.3)$ & 190.06 & 0.1 & 193.14 & 0.1 & 3.08 & 0.14 \\
\hline $\operatorname{Exp} 4(15,14.9)$ & 190.06 & 0.1 & 195.42 & 0.1 & 5.36 & 0.14 \\
\hline $\operatorname{Exp} 4(30,7.4)$ & 190.06 & 0.1 & 190.96 & 0.1 & 0.90 & 0.14 \\
\hline $\operatorname{Exp} 4(30,9.8)$ & 190.06 & 0.1 & 191.67 & 0.1 & 1.61 & 0.14 \\
\hline Exp4(30, 11.0) & 190.06 & 0.1 & 192.38 & 0.1 & 2.32 & 0.14 \\
\hline $\operatorname{Exp} 4(30,12.3)$ & 190.06 & 0.1 & 193.27 & 0.1 & 3.21 & 0.14 \\
\hline $\operatorname{Exp} 4(30,14.9)$ & 190.06 & 0.1 & 196.83 & 0.1 & 6.77 & 0.14 \\
\hline $\operatorname{Exp} 5(15,7.4)$ & 190.08 & 0.1 & 190.47 & 0.1 & 0.39 & 0.14 \\
\hline $\operatorname{Exp} 5(15,9.8)$ & 190.08 & 0.1 & 191.90 & 0.1 & 1.82 & 0.14 \\
\hline $\operatorname{Exp} 5(15,12.3)$ & 190.08 & 0.1 & 192.90 & 0.1 & 2.82 & 0.14 \\
\hline $\operatorname{Exp} 5(15,14.9)$ & 190.08 & 0.1 & 194.27 & 0.1 & 4.19 & 0.14 \\
\hline
\end{tabular}




\section{Appendix B - Experimental ICP-MS Concentration Data}

\begin{tabular}{|c|c|c|c|c|c|}
\hline Experiment & Salt Region & $\begin{array}{c}\mathrm{Li} \\
\text { Concentration } \\
(\mu \mathrm{g} / \mathrm{L})\end{array}$ & \pm & $\begin{array}{c}\text { Cs } \\
\text { Concentration } \\
(\mu \mathrm{g} / \mathrm{L})\end{array}$ & \pm \\
\hline $\operatorname{Exp} 2(15,7.4)$ & Bulk & 6,219 & $10 \%$ & 206 & $10 \%$ \\
\hline $\operatorname{Exp} 2(15,7.4)$ & Drip & 942 & $10 \%$ & 102 & $10 \%$ \\
\hline $\operatorname{Exp} 2(15,7.4)$ & Crystal & 5174 & $10 \%$ & 0 & $10 \%$ \\
\hline $\operatorname{Exp} 2(15,9.8)$ & Bulk & 5764 & $10 \%$ & 210 & $10 \%$ \\
\hline $\operatorname{Exp} 2(15,9.8)$ & Drip & 1937 & $10 \%$ & 132 & $10 \%$ \\
\hline $\operatorname{Exp} 2(15,9.8)$ & Crystal & 5767 & $10 \%$ & 0 & $10 \%$ \\
\hline $\operatorname{Exp} 2(15,12.3)$ & Bulk & 5680 & $10 \%$ & 218 & $10 \%$ \\
\hline $\operatorname{Exp} 2(15,12.3)$ & Drip & 2205 & $10 \%$ & 135 & $10 \%$ \\
\hline $\operatorname{Exp} 2(15,12.3)$ & Crystal & 5860 & $10 \%$ & 0 & $10 \%$ \\
\hline $\operatorname{Exp} 2(15,14.9)$ & Bulk & 5812 & $10 \%$ & 239 & $10 \%$ \\
\hline $\operatorname{Exp} 2(15,14.9)$ & Drip & 2785 & $10 \%$ & 153 & $10 \%$ \\
\hline $\operatorname{Exp} 2(15,14.9)$ & Crystal & 5756 & $10 \%$ & 0 & $10 \%$ \\
\hline $\operatorname{Exp} 3(15,7.4)$ & Bulk & 5679 & $10 \%$ & 733 & $10 \%$ \\
\hline $\operatorname{Exp} 3(15,7.4)$ & Drip & 1513 & $10 \%$ & 231 & $10 \%$ \\
\hline $\operatorname{Exp} 3(15,7.4)$ & Crystal & 5307 & $10 \%$ & 56.8 & $10 \%$ \\
\hline $\operatorname{Exp} 3(15,9.8)$ & Bulk & 5523 & $10 \%$ & 887 & $10 \%$ \\
\hline $\operatorname{Exp} 3(15,9.8)$ & Drip & 1818 & $10 \%$ & 209 & $10 \%$ \\
\hline $\operatorname{Exp} 3(15,9.8)$ & Crystal & 5046 & $10 \%$ & 13.0 & $10 \%$ \\
\hline $\operatorname{Exp} 3(15,12.3)$ & Bulk & 5479 & $10 \%$ & 905 & $10 \%$ \\
\hline $\operatorname{Exp} 3(15,12.3)$ & Drip & 2759 & $10 \%$ & 298 & $10 \%$ \\
\hline $\operatorname{Exp} 3(15,12.3)$ & Crystal & 5657 & $10 \%$ & 16.5 & $10 \%$ \\
\hline Exp3(15, 14.9) & Bulk & 5068 & $10 \%$ & 997 & $10 \%$ \\
\hline
\end{tabular}




\begin{tabular}{|c|c|c|c|c|c|}
\hline $\operatorname{Exp} 3(15,14.9)$ & Drip & 1968 & $10 \%$ & 256 & $10 \%$ \\
\hline $\operatorname{Exp} 3(15,14.9)$ & Crystal & 5332 & $10 \%$ & 14.0 & $10 \%$ \\
\hline $\operatorname{Exp} 4(5,7.4)$ & Bulk & 5018 & $10 \%$ & 1297 & $10 \%$ \\
\hline $\operatorname{Exp} 4(5,7.4)$ & Crystal + Drip & 5454 & $10 \%$ & 297 & $10 \%$ \\
\hline $\operatorname{Exp} 4(5,9.8)$ & Bulk & 5224 & $10 \%$ & 1319 & $10 \%$ \\
\hline $\operatorname{Exp} 4(5,9.8)$ & Crystal + Drip & 5075 & $10 \%$ & 107 & $10 \%$ \\
\hline $\operatorname{Exp} 4(5,12.3)$ & Bulk & 4766 & $10 \%$ & 1334 & $10 \%$ \\
\hline $\operatorname{Exp} 4(5,12.3)$ & Crystal + Drip & 5256 & $10 \%$ & 148 & $10 \%$ \\
\hline $\operatorname{Exp} 4(5,14.9)$ & Bulk & 4630 & $10 \%$ & 1264 & $10 \%$ \\
\hline $\operatorname{Exp} 4(5,14.9)$ & Crystal + Drip & 5077 & $10 \%$ & 130 & $10 \%$ \\
\hline $\operatorname{Exp} 4(10,7.4)$ & Bulk & 3998 & $10 \%$ & 1069 & $10 \%$ \\
\hline $\operatorname{Exp} 4(10,7.4)$ & Crystal + Drip & 4972 & $10 \%$ & 139 & $10 \%$ \\
\hline $\operatorname{Exp} 4(10,9.8)$ & Bulk & 4702 & $10 \%$ & 1302 & $10 \%$ \\
\hline $\operatorname{Exp} 4(10,9.8)$ & Crystal + Drip & 5290 & $10 \%$ & 91.2 & $10 \%$ \\
\hline $\operatorname{Exp} 4(10,12.3)$ & Bulk & 4574 & $10 \%$ & 1333 & $10 \%$ \\
\hline $\operatorname{Exp} 4(10,12.3)$ & Crystal + Drip & 5226 & $10 \%$ & 96.7 & $10 \%$ \\
\hline $\operatorname{Exp} 4(10,14.9)$ & Bulk & 4567 & $10 \%$ & 1341 & $10 \%$ \\
\hline Exp4(10, 14.9) & Crystal + Drip & 5016 & $10 \%$ & 88.2 & $10 \%$ \\
\hline $\operatorname{Exp} 4(15,7.4)$ & Bulk & 4746 & $10 \%$ & 1275 & $10 \%$ \\
\hline Exp4(15, 7.4) & Crystal + Drip & 4987 & $10 \%$ & 106 & $10 \%$ \\
\hline Exp4(15, 9.8) & Bulk & 4700 & $10 \%$ & 1335 & $10 \%$ \\
\hline Exp4(15, 9.8) & Crystal + Drip & 5296 & $10 \%$ & 64.2 & $10 \%$ \\
\hline $\operatorname{Exp} 4(15,12.3)$ & Bulk & 4978 & $10 \%$ & 1378 & $10 \%$ \\
\hline $\operatorname{Exp} 4(15,12.3)$ & Crystal + Drip & 5259 & $10 \%$ & 70.5 & $10 \%$ \\
\hline $\operatorname{Exp} 4(15,14.9)$ & Bulk & 4692 & $10 \%$ & 1560 & $10 \%$ \\
\hline
\end{tabular}




\begin{tabular}{|c|c|c|c|c|c|}
\hline Exp4(15, 14.9) & Crystal + Drip & 4788 & $10 \%$ & 76.5 & $10 \%$ \\
\hline $\operatorname{Exp} 4(30,7.4)$ & Bulk & 4683 & $10 \%$ & 1285 & $10 \%$ \\
\hline $\operatorname{Exp} 4(30,7.4)$ & Crystal + Drip & 5011 & $10 \%$ & 146 & $10 \%$ \\
\hline Exp4(30, 9.8) & Bulk & 5019 & $10 \%$ & 1321 & $10 \%$ \\
\hline Exp4(30, 9.8) & Crystal + Drip & 5093 & $10 \%$ & 74.6 & $10 \%$ \\
\hline $\operatorname{Exp} 4(30,11.0)$ & Bulk & 4787 & $10 \%$ & 1266 & $10 \%$ \\
\hline $\operatorname{Exp} 4(30,11.0)$ & Crystal + Drip & 5590 & $10 \%$ & 37.0 & $10 \%$ \\
\hline $\operatorname{Exp} 4(30,12.3)$ & Bulk & 5259 & $10 \%$ & 1199 & $10 \%$ \\
\hline $\operatorname{Exp} 4(30,12.3)$ & Crystal + Drip & 5268 & $10 \%$ & 77.2 & $10 \%$ \\
\hline $\operatorname{Exp} 4(30,14.9)$ & Bulk & 4694 & $10 \%$ & 1542 & $10 \%$ \\
\hline $\operatorname{Exp} 4(30,14.9)$ & Crystal + Drip & 5064 & $10 \%$ & 68.2 & $10 \%$ \\
\hline $\operatorname{Exp} 5(15,7.4)$ & Bulk & 4960 & $10 \%$ & 1927 & $10 \%$ \\
\hline $\operatorname{Exp} 5(15,7.4)$ & Drip & 353 & $10 \%$ & 241 & $10 \%$ \\
\hline $\operatorname{Exp} 5(15,7.4)$ & Crystal & 5491 & $10 \%$ & 558 & $10 \%$ \\
\hline $\operatorname{Exp} 5(15,9.8)$ & Bulk & 4671 & $10 \%$ & 1903 & $10 \%$ \\
\hline $\operatorname{Exp5}(15,9.8)$ & Drip & 638 & $10 \%$ & 132 & $10 \%$ \\
\hline $\operatorname{Exp} 5(15,9.8)$ & Crystal & 5091 & $10 \%$ & 79.6 & $10 \%$ \\
\hline $\operatorname{Exp} 5(15,12.3)$ & Bulk & 5261 & $10 \%$ & 2260 & $10 \%$ \\
\hline $\operatorname{Exp} 5(15,12.3)$ & Drip & 1043 & $10 \%$ & 265 & $10 \%$ \\
\hline $\operatorname{Exp} 5(15,12.3)$ & Crystal & 5205 & $10 \%$ & 13.0 & $30 \%$ \\
\hline $\operatorname{Exp5}(15,14.9)$ & Bulk & 4853 & $10 \%$ & 2159 & $10 \%$ \\
\hline $\operatorname{Exp5}(15,14.9)$ & Drip & 1107 & $10 \%$ & 216 & $10 \%$ \\
\hline $\operatorname{Exp} 5(15,14.9)$ & Crystal & 4454 & $10 \%$ & 14.9 & $30 \%$ \\
\hline
\end{tabular}




\section{Appendix C - Calculated Parameters of Dimensionless Groups}

\begin{tabular}{|c|c|c|c|c|c|c|c|c|c|}
\hline Experiment & $\begin{array}{c}L^{3} \\
\left(\mathrm{~m}^{3}\right)\end{array}$ & $\begin{array}{c}T_{\text {Freeze }} \\
(\mathrm{K})\end{array}$ & $\begin{array}{c}T_{\text {Bulk }} \\
(\mathrm{K})\end{array}$ & $\begin{array}{c}k \\
(\mathrm{~W} / \mathrm{m} \mathrm{K})\end{array}$ & $\begin{array}{c}\beta \\
\left(\mathrm{K}^{-1}\right)\end{array}$ & $\begin{array}{c}\rho \\
\left(\mathrm{kg} / \mathrm{m}^{3}\right)\end{array}$ & $\begin{array}{c}\mu \\
(\mathrm{kg} / \mathrm{s} \mathrm{m})\end{array}$ & $\begin{array}{c}C_{P} \\
(\mathrm{~J} / \mathrm{kg} \mathrm{K})\end{array}$ & $\begin{array}{c}D_{A B} \\
\left(\mathrm{~m}^{2} / \mathrm{s}\right)\end{array}$ \\
\hline $\operatorname{Exp} 2(15,7.4)$ & 4.93E-07 & 882 & 909 & 0.624 & 0.000289 & 1497 & 0.00141 & 1535 & $2.50 \mathrm{E}-09$ \\
\hline $\operatorname{Exp} 2(15,9.8)$ & $1.28 \mathrm{E}-06$ & 882 & 901 & 0.626 & 0.000289 & 1498 & 0.00143 & 1536 & $2.46 \mathrm{E}-09$ \\
\hline $\operatorname{Exp} 2(15,12.3)$ & $2.37 \mathrm{E}-06$ & 882 & 895 & 0.627 & 0.000289 & 1500 & 0.00144 & 1537 & $2.43 \mathrm{E}-09$ \\
\hline $\operatorname{Exp} 2(15,14.9)$ & $3.07 \mathrm{E}-06$ & 882 & 895 & 0.627 & 0.000289 & 1500 & 0.00144 & 1537 & $2.43 \mathrm{E}-09$ \\
\hline $\operatorname{Exp} 3(15,7.4)$ & $3.53 \mathrm{E}-07$ & 880 & 910 & 0.624 & 0.000289 & 1497 & 0.00141 & 1535 & $2.49 \mathrm{E}-09$ \\
\hline $\operatorname{Exp} 3(15,9.8)$ & $1.24 \mathrm{E}-06$ & 880 & 899 & 0.627 & 0.000289 & 1499 & 0.00144 & 1536 & $2.44 \mathrm{E}-09$ \\
\hline $\operatorname{Exp} 3(15,12.3)$ & $1.76 \mathrm{E}-06$ & 880 & 898 & 0.627 & 0.000289 & 1499 & 0.00144 & 1536 & $2.43 \mathrm{E}-09$ \\
\hline $\operatorname{Exp} 3(15,14.9)$ & $2.73 \mathrm{E}-06$ & 880 & 894 & 0.628 & 0.000288 & 1500 & 0.00145 & 1537 & $2.41 \mathrm{E}-09$ \\
\hline $\operatorname{Exp} 4(5,7.4)$ & $2.61 \mathrm{E}-07$ & 878 & 915 & 0.624 & 0.000289 & 1496 & 0.00141 & 1535 & $2.51 \mathrm{E}-09$ \\
\hline Exp4(5, 9.8) & $5.27 \mathrm{E}-07$ & 878 & 912 & 0.624 & 0.000289 & 1497 & 0.00141 & 1535 & $2.49 \mathrm{E}-09$ \\
\hline Exp4(5, 12.3) & $8.12 \mathrm{E}-07$ & 878 & 905 & 0.626 & 0.000289 & 1498 & 0.00143 & 1536 & $2.46 \mathrm{E}-09$ \\
\hline $\operatorname{Exp} 4(5,14.9)$ & $9.77 \mathrm{E}-07$ & 878 & 904 & 0.626 & 0.000289 & 1498 & 0.00143 & 1536 & $2.45 \mathrm{E}-09$ \\
\hline Exp4(10, 7.4) & $3.68 \mathrm{E}-07$ & 878 & 910 & 0.625 & 0.000289 & 1497 & 0.00142 & 1535 & $2.48 \mathrm{E}-09$ \\
\hline Exp4(10, 9.8) & $8.51 \mathrm{E}-07$ & 878 & 902 & 0.626 & 0.000289 & 1499 & 0.00143 & 1536 & $2.44 \mathrm{E}-09$ \\
\hline $\operatorname{Exp} 4(10,12.3)$ & $1.26 \mathrm{E}-06$ & 878 & 899 & 0.627 & 0.000289 & 1500 & 0.00144 & 1537 & $2.43 \mathrm{E}-09$ \\
\hline $\operatorname{Exp} 4(10,14.9)$ & $1.60 \mathrm{E}-06$ & 878 & 896 & 0.628 & 0.000288 & 1500 & 0.00145 & 1537 & $2.41 \mathrm{E}-09$ \\
\hline Exp4(15, 7.4) & $4.11 \mathrm{E}-07$ & 878 & 909 & 0.625 & 0.000289 & 1497 & 0.00142 & 1535 & $2.48 \mathrm{E}-09$ \\
\hline Exp4(15, 9.8) & $8.46 \mathrm{E}-07$ & 878 & 900 & 0.627 & 0.000289 & 1499 & 0.00144 & 1536 & $2.43 \mathrm{E}-09$ \\
\hline $\operatorname{Exp} 4(15,12.3)$ & $1.49 \mathrm{E}-06$ & 878 & 897 & 0.627 & 0.000289 & 1500 & 0.00145 & 1537 & $2.42 \mathrm{E}-09$ \\
\hline Exp4(15, 14.9) & $2.59 \mathrm{E}-06$ & 878 & 890 & 0.629 & 0.000288 & 1501 & 0.00146 & 1537 & $2.38 \mathrm{E}-09$ \\
\hline $\operatorname{Exp} 4(30,7.4)$ & 4.35E-07 & 878 & 909 & 0.625 & 0.000289 & 1497 & 0.00142 & 1535 & $2.48 \mathrm{E}-09$ \\
\hline Exp4(30, 9.8) & 7.79E-07 & 878 & 902 & 0.626 & 0.000289 & 1499 & 0.00143 & 1536 & $2.44 \mathrm{E}-09$ \\
\hline $\operatorname{Exp} 4(30,11.0)$ & $1.12 \mathrm{E}-06$ & 878 & 900 & 0.627 & 0.000289 & 1499 & 0.00144 & 1536 & $2.43 \mathrm{E}-09$ \\
\hline $\operatorname{Exp} 4(30,12.3)$ & $1.55 \mathrm{E}-06$ & 878 & 896 & 0.628 & 0.000288 & 1500 & 0.00145 & 1537 & $2.41 \mathrm{E}-09$ \\
\hline $\operatorname{Exp} 4(30,14.9)$ & $3.27 \mathrm{E}-06$ & 878 & 889 & 0.629 & 0.000288 & 1502 & 0.00146 & 1538 & $2.38 \mathrm{E}-09$ \\
\hline $\operatorname{Exp} 5(15,7.4)$ & $1.89 \mathrm{E}-07$ & 876 & 910 & 0.625 & 0.000289 & 1498 & 0.00142 & 1536 & 2.47E-09 \\
\hline
\end{tabular}




\begin{tabular}{rrrrrrrrrr}
$\operatorname{Exp} 5(15,9.8)$ & $8.80 \mathrm{E}-07$ & 876 & 898 & 0.628 & 0.000288 & 1500 & 0.00145 & 1537 & $2.41 \mathrm{E}-09$ \\
$\operatorname{Exp} 5(15,12.3)$ & $1.36 \mathrm{E}-06$ & 876 & 899 & 0.628 & 0.000289 & 1500 & 0.00145 & 1537 & $2.41 \mathrm{E}-09$ \\
$\operatorname{Exp} 5(15,14.9)$ & $2.03 \mathrm{E}-06$ & 876 & 895 & 0.628 & 0.000288 & 1501 & 0.00145 & 1537 & $2.39 \mathrm{E}-09$ \\
\hline
\end{tabular}




\section{Appendix D - Experimental Dimensionless Correlation Parameters}

\begin{tabular}{|c|c|c|c|c|c|c|}
\hline Experiment & $G r$ & $\operatorname{Pr}$ & $S c$ & $G r \operatorname{Pr}$ & $G r S c$ & $G r \operatorname{Pr} S c$ \\
\hline $\operatorname{Exp} 2(15,7.4)$ & 42268 & 3.47 & 378 & 146809 & 15988078 & 55530489 \\
\hline $\operatorname{Exp} 2(15,9.8)$ & 75218 & 3.51 & 388 & 263748 & 29217959 & 102450603 \\
\hline $\operatorname{Exp} 2(15,12.3)$ & 93949 & 3.53 & 396 & 331805 & 37234614 & 131504272 \\
\hline $\operatorname{Exp} 2(15,14.9)$ & 121502 & 3.53 & 396 & 429116 & 48154745 & 170071717 \\
\hline $\operatorname{Exp} 3(15,7.4)$ & 33396 & 3.48 & 379 & 116113 & 12668535 & 44046126 \\
\hline $\operatorname{Exp} 3(15,9.8)$ & 71665 & 3.52 & 394 & 252457 & 28200476 & 99342497 \\
\hline $\operatorname{Exp} 3(15,12.3)$ & 95943 & 3.53 & 395 & 338388 & 37880910 & 133604614 \\
\hline $\operatorname{Exp} 3(15,14.9)$ & 114466 & 3.54 & 400 & 405673 & 45806897 & 162341344 \\
\hline $\operatorname{Exp} 4(5,7.4)$ & 30705 & 3.46 & 376 & 106370 & 11530011 & 39942507 \\
\hline $\operatorname{Exp} 4(5,9.8)$ & 56452 & 3.48 & 379 & 196255 & 21409174 & 74429237 \\
\hline $\operatorname{Exp} 4(5,12.3)$ & 67633 & 3.51 & 388 & 237092 & 26253493 & 92033411 \\
\hline Exp4(5, 14.9) & 78061 & 3.51 & 389 & 273976 & 30402825 & 106706711 \\
\hline Exp4(10, 7.4) & 36824 & 3.48 & 382 & 128323 & 14058354 & 48989975 \\
\hline Exp4(10, 9.8) & 62378 & 3.52 & 392 & 219458 & 24457863 & 86047307 \\
\hline Exp4(10, 12.3) & 79826 & 3.53 & 396 & 281856 & 31615491 & 111631253 \\
\hline Exp4(10, 14.9) & 85922 & 3.54 & 400 & 304485 & 34375787 & 121818257 \\
\hline Exp4(15, 7.4) & 39777 & 3.49 & 383 & 138779 & 15236273 & 53157818 \\
\hline Exp4(15, 9.8) & 56479 & 3.53 & 395 & 199182 & 22293937 & 78622984 \\
\hline Exp4(15, 12.3) & 84958 & 3.54 & 399 & 300704 & 33875572 & 119900499 \\
\hline Exp4(15, 14.9) & 90745 & 3.57 & 408 & 323929 & 37050152 & 132256777 \\
\hline $\operatorname{Exp} 4(30,7.4)$ & 42117 & 3.49 & 383 & 146942 & 16132525 & 56284749 \\
\hline
\end{tabular}




\begin{tabular}{|lllllll}
$\operatorname{Exp} 4(30,9.8)$ & 57062 & 3.52 & 392 & 200754 & 22373386 & 78713729 \\
\hline $\operatorname{Exp} 4(30,11.0)$ & 74875 & 3.53 & 395 & 264059 & 29555391 & 104231613 \\
\hline $\operatorname{Exp} 4(30,12.3)$ & 83579 & 3.54 & 400 & 296181 & 33438265 & 118495941 \\
\hline $\operatorname{Exp} 4(30,14.9)$ & 104534 & 3.57 & 410 & 373609 & 42825628 & 153060236 \\
\hline $\operatorname{Exp} 5(15,7.4)$ & 20245 & 3.50 & 385 & 70757 & 7793104 & 27237722 \\
\hline $\operatorname{Exp} 5(15,9.8)$ & 59333 & 3.55 & 401 & 210384 & 23777376 & 84310884 \\
\hline $\operatorname{Exp} 5(15,12.3)$ & 96341 & 3.54 & 399 & 341195 & 38478156 & 136272498 \\
\hline $\operatorname{Exp} 5(15,14.9)$ & 117157 & 3.56 & 405 & 416937 & 47429155 & 168790078 \\
\hline
\end{tabular}


Appendix E - Thesis Defense Slides

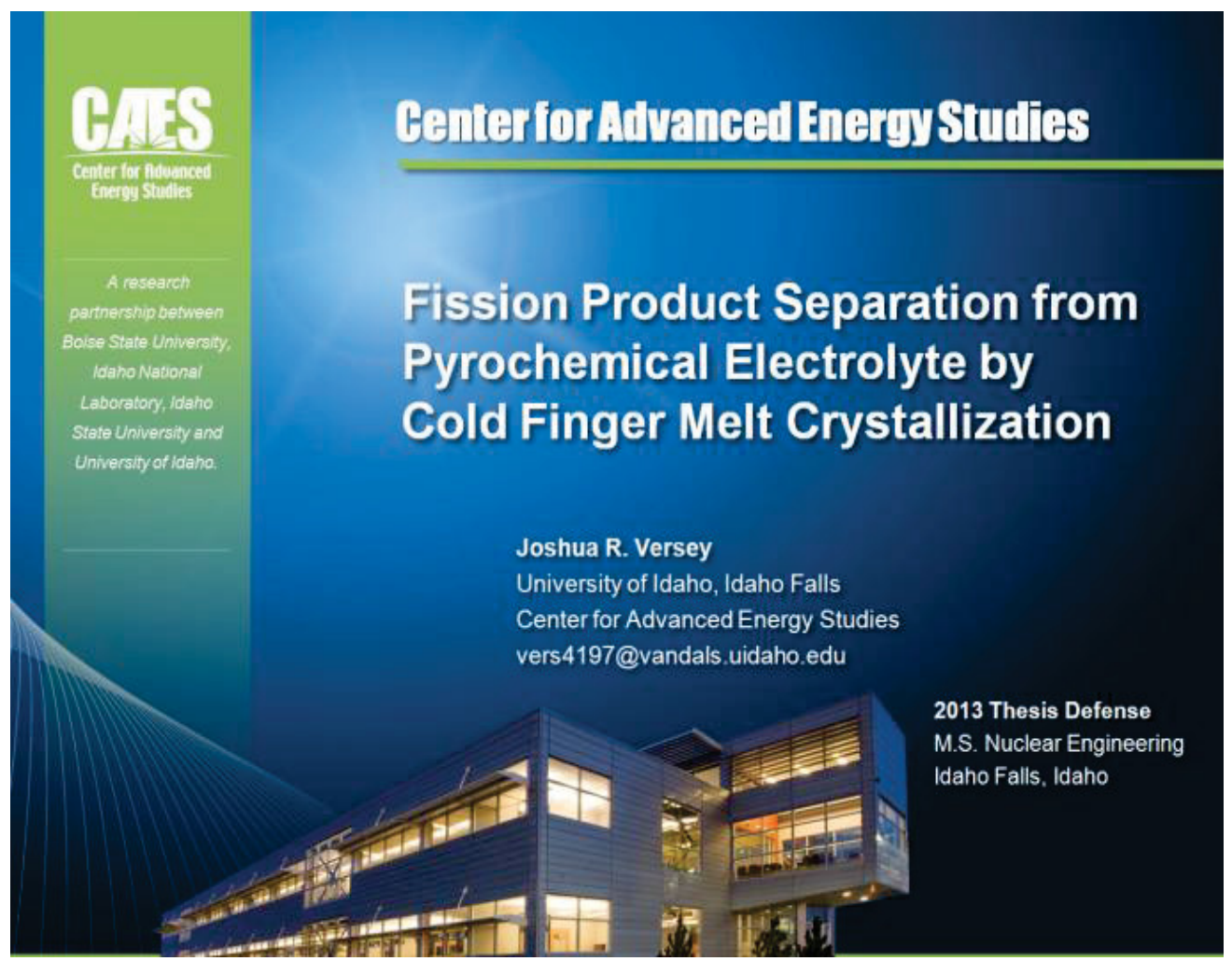

\section{Outline}

Gers

- Background

- Motivation \& Goals

- Cold Finger Theory

- Cold Finger Design

- Experimental Program

- Results \& Discussion

- Experimental

- Crystal Growth Correlations

- Summary \& Future Work 


\section{Background}

\section{Advanced Pyrochemical Technology Concept}

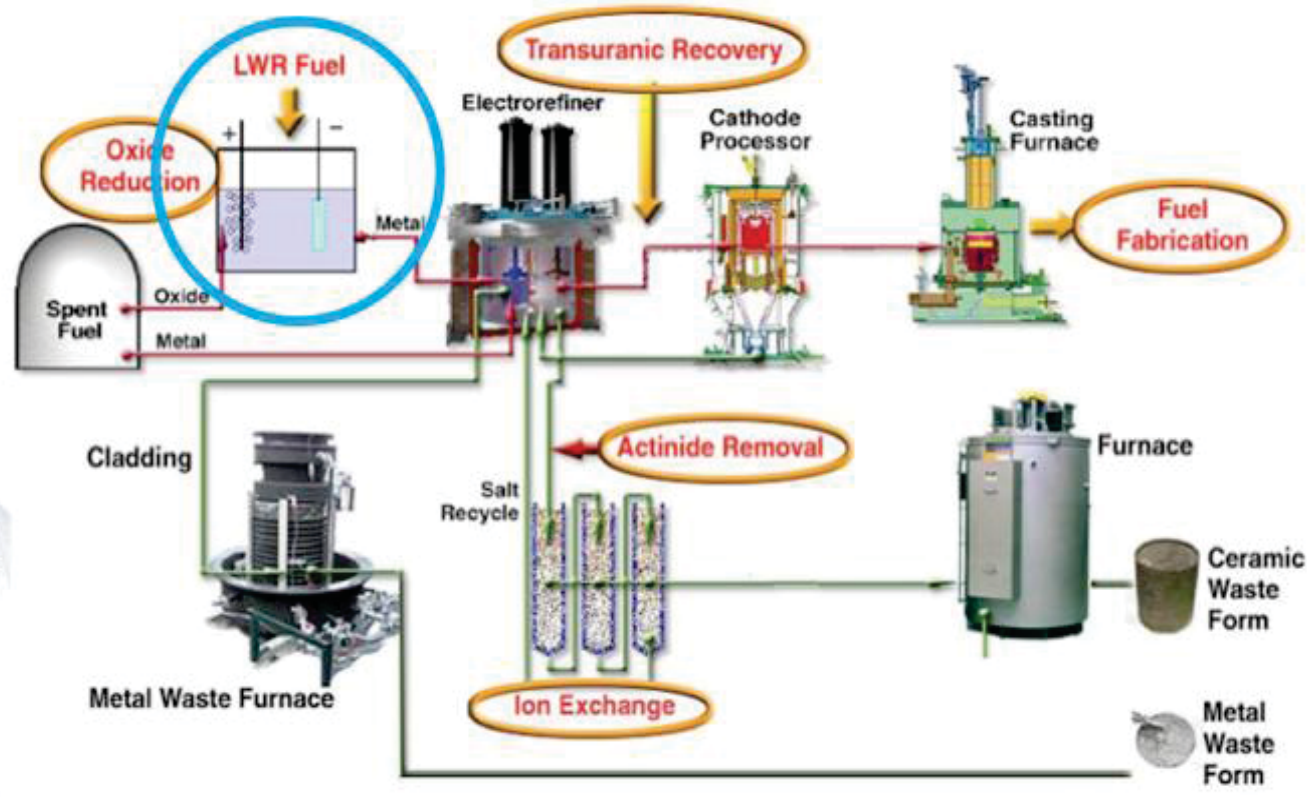

\section{Background}

\section{Oxide Reduction Process}

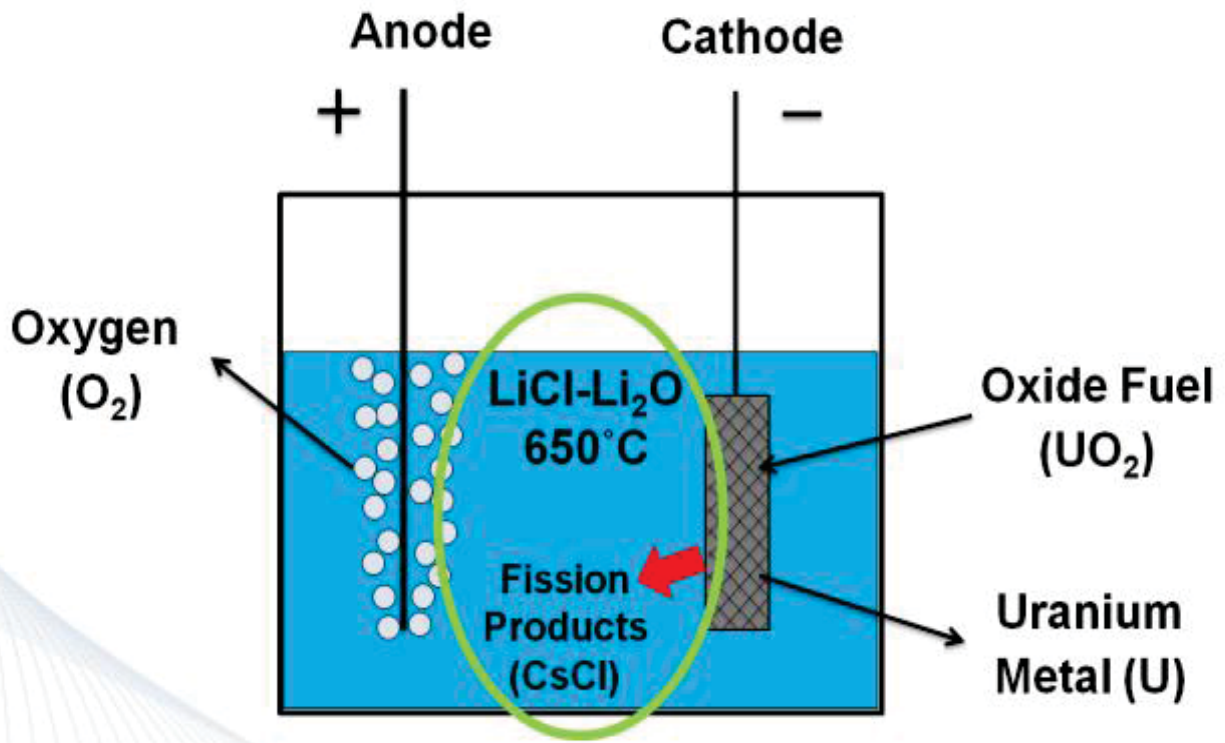

- Fission product build-up effects: electrolyte characteristics and process efficiencies 


\section{Motivation \& Goals}

- Methods for cleaning used electrolyte are needed to reduce waste volume and cost

- Melt crystallization techniques have been explored:

- Zone freezing (KAERI and $U$ of $I$ )

- Layer crystallization (KAERI)

- A cold finger technique has been proposed to study the effect of fundamental parameters on separation

- Initial $\mathrm{CsCl}$ concentration

- Crystal growth time

- Cooling gas flow rate

- Result will provide information on optimal operating parameters and conditions

\section{Cold Finger Theory}

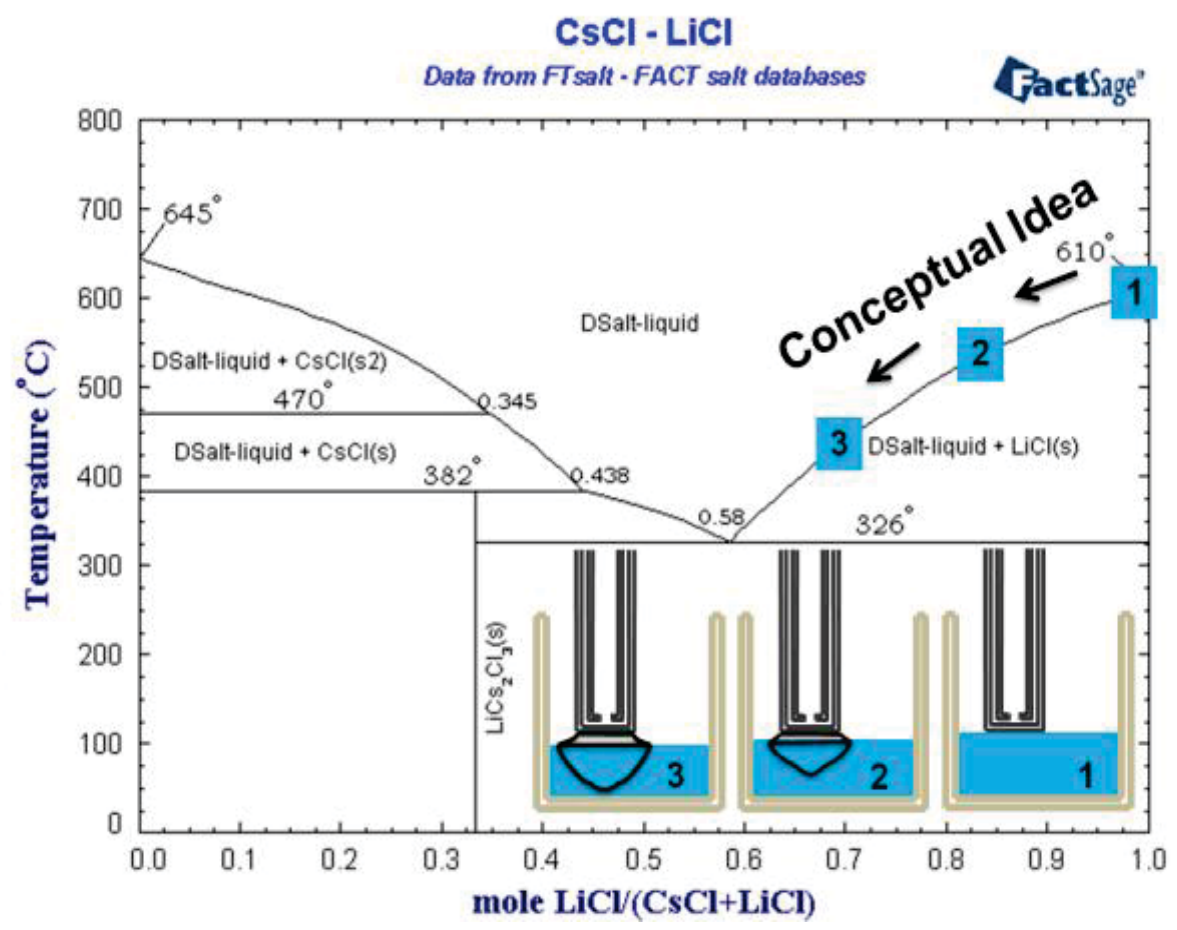




\section{Cold Finger Design}

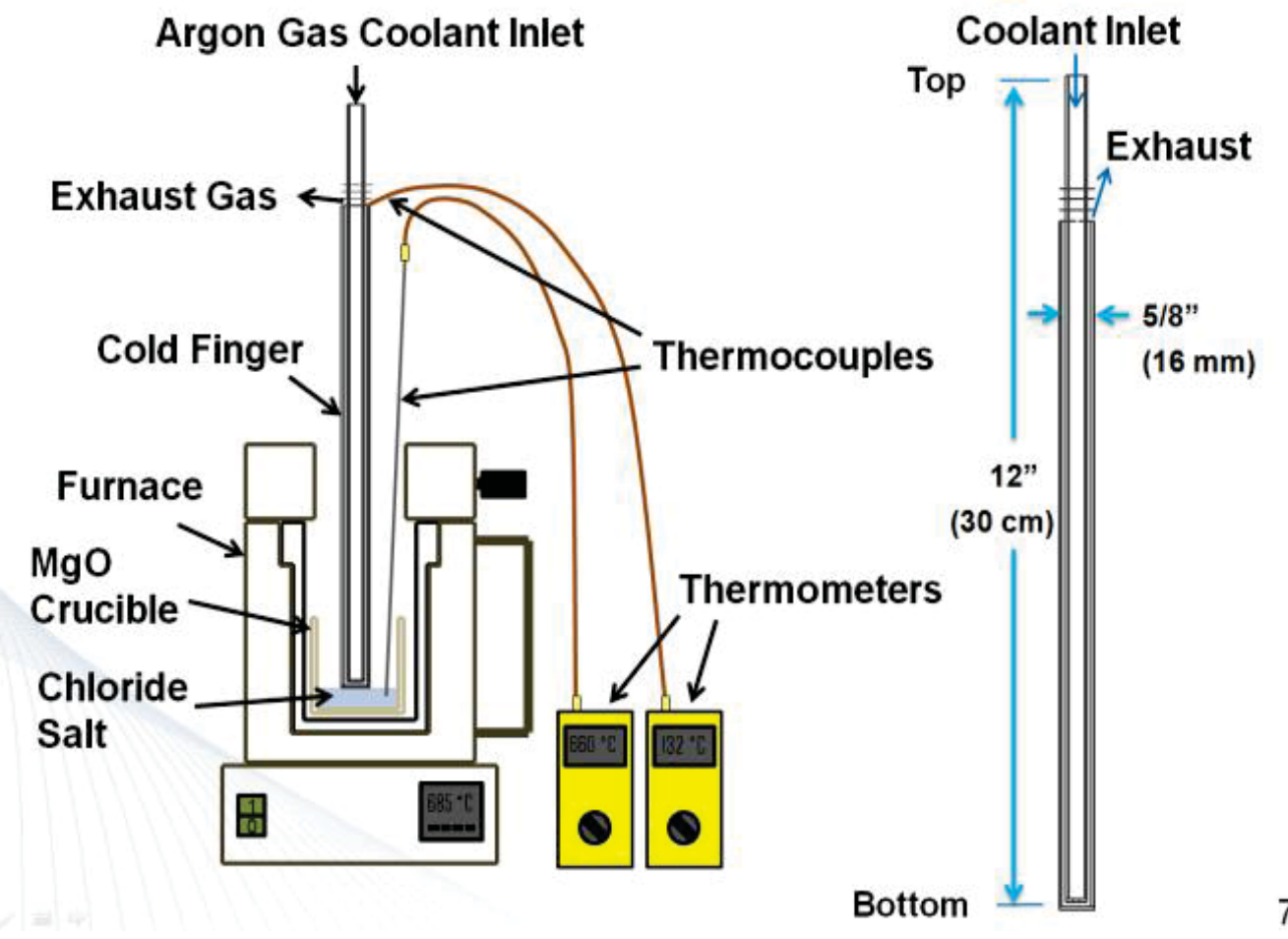

\section{Experimental Setup}
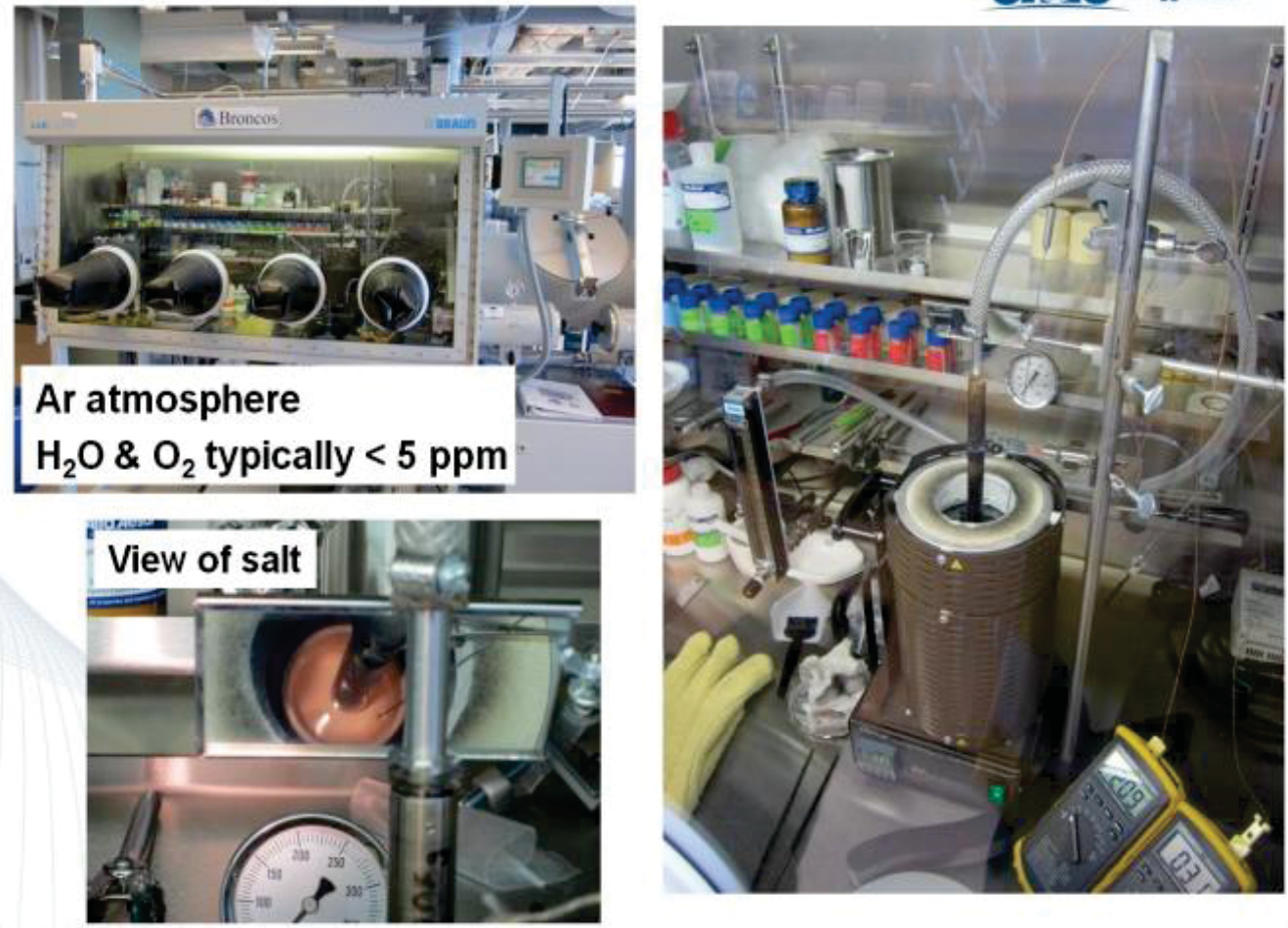


\section{Experimental Procedure}

- Loaded $30 \mathrm{~g}$ of LiCl-CsCl in MgO crucible - $0,1,3,5$, and $7.5 \mathrm{wt} \% \mathrm{CsCl}$

- Melted salt at furnace temperature of $685^{\circ} \mathrm{C}$

- Positioned cold finger at top surface of molten salt

- Experiments began once salt temperature reached $660^{\circ} \mathrm{C}\left( \pm 3^{\circ} \mathrm{C}\right)$

- Varied argon cooling gas flow rate and duration for ranges within

- 5.1 to $14.9 \mathrm{~L} / \mathrm{min}$

- 1 to $60 \mathrm{~min}$

\section{Sample Preparation/Analysis}

- Crystal

- Weighed

- Dimensioned

- Crushed

- Homogenized

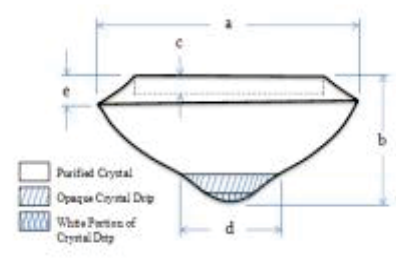

- Analyzed by ICP-MS

- Li \& Cs concentrations

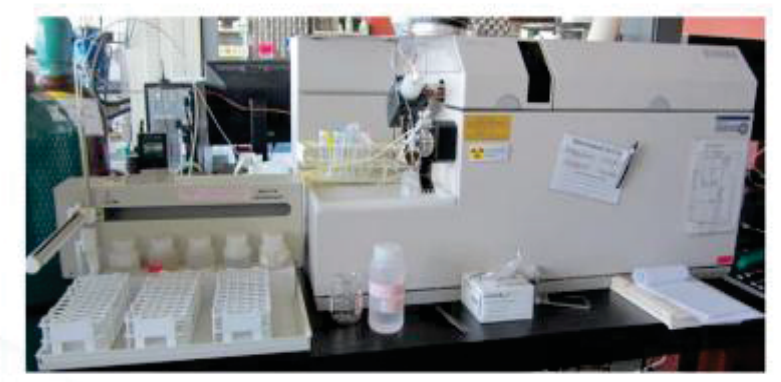




\section{Experiments Performed}

\begin{tabular}{cccc}
\hline $\begin{array}{c}\text { Exp. } \\
\text { No. }\end{array}$ & $\begin{array}{c}\text { Initial LiCl-CsCl } \\
\text { Concentration } \\
\text { (wt\% CsCl) }\end{array}$ & $\begin{array}{c}\text { Crystal Growth } \\
\text { Time (min) }\end{array}$ & $\begin{array}{c}\text { Cooling Gas } \\
\text { Flow Rate } \\
(\text { L/min) }\end{array}$ \\
\hline 1a & 0 & $1-60$ & 11.0 \\
\hline 1b & 0 & 30 & $5.1-11.0$ \\
\hline 2 & 1 & 15 & $7.4-14.9$ \\
\hline 3 & 3 & 15 & $7.4-14.9$ \\
\hline 4 & 5 & $5-30$ & $7.4-14.9$ \\
5 & 7.5 & 15 & $7.4-14.9$ \\
\hline
\end{tabular}

Note: $1 \mathrm{~L} / \mathrm{min}=1.667 \times 10^{-5} \mathrm{~m}^{3} / \mathrm{s}$

\section{Duration Effect}

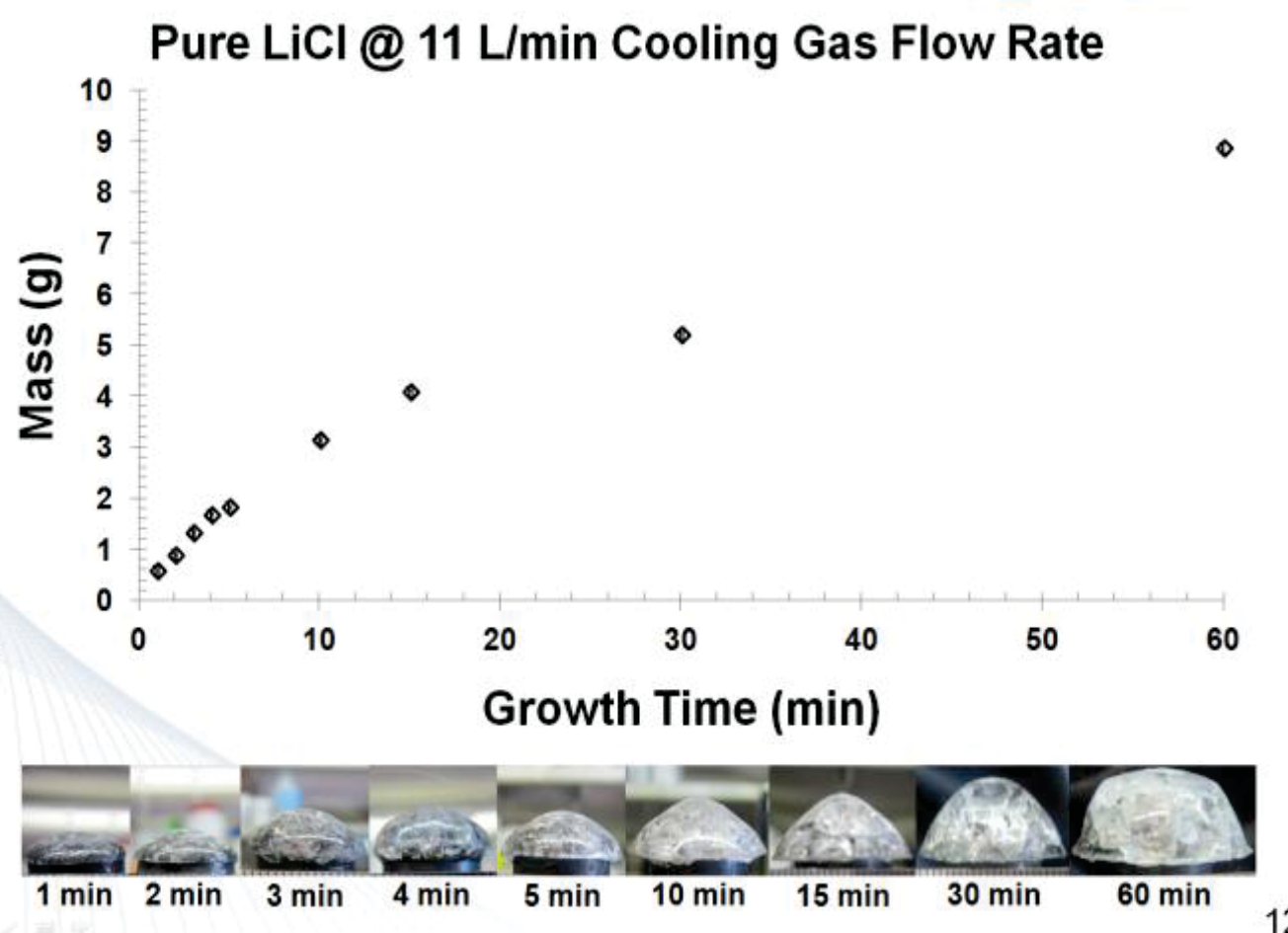




\section{Cooling Rate Effect}

1. Center for Rduanced

Pure LiCl @ 30 min Growth Time
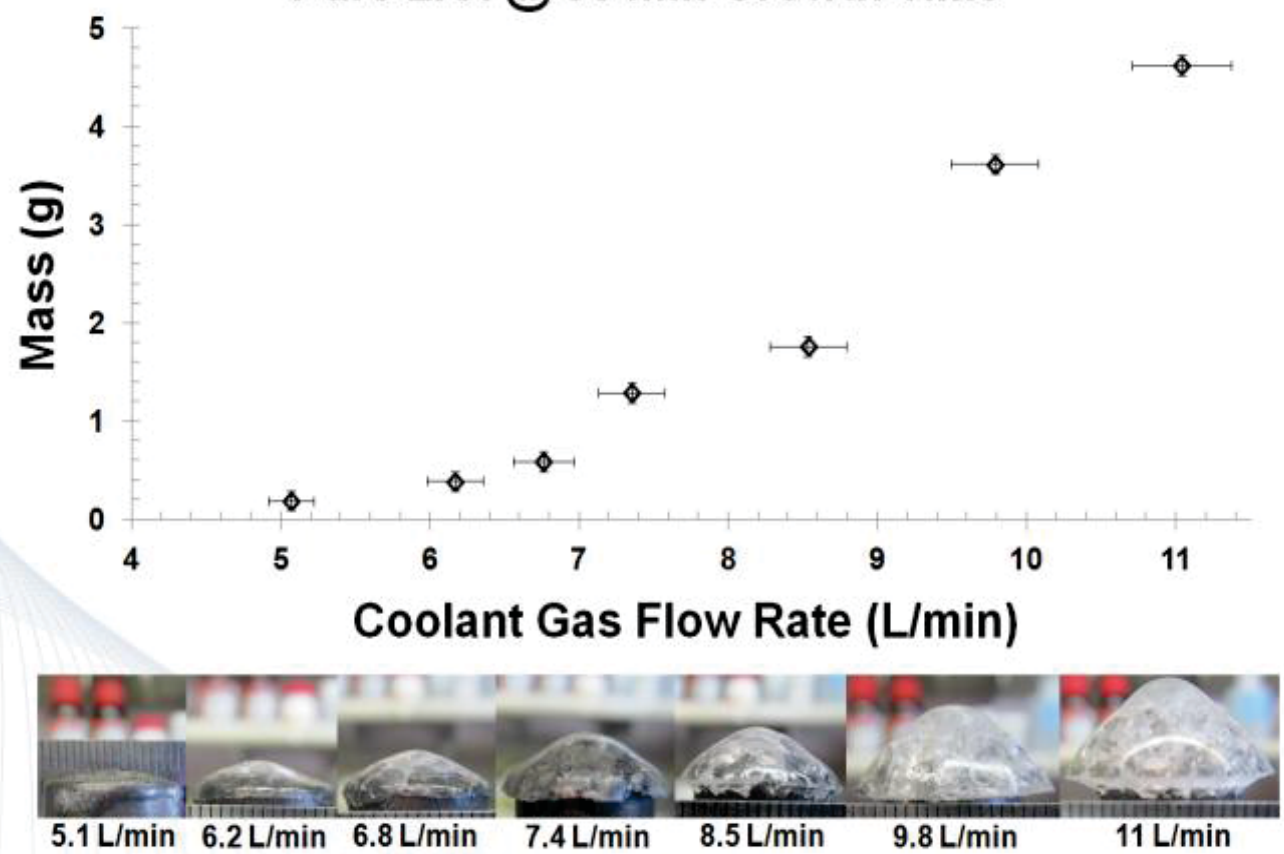

\section{Crystal Formation Matrix}

$\mathrm{LiCl}-\mathrm{CsCl}$ (Initial 5 wt\% CsCl)

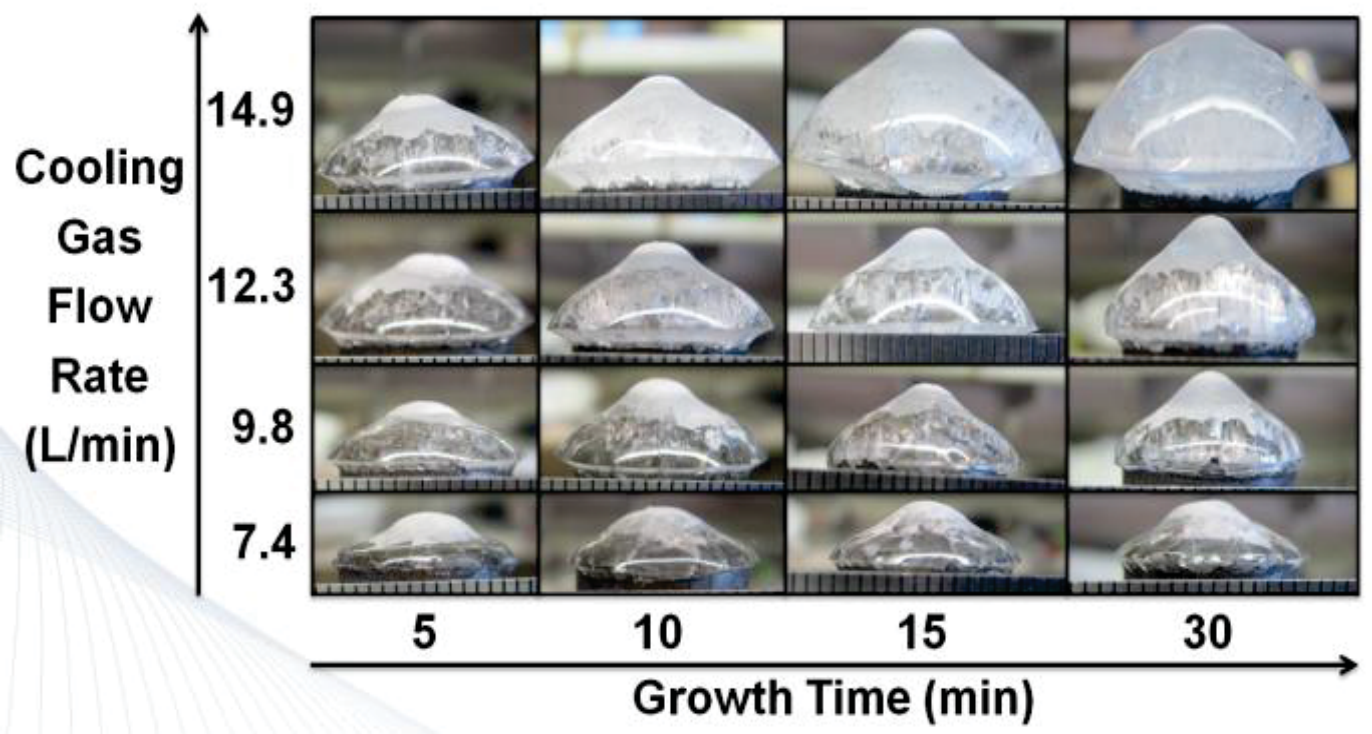




\section{Concentration Data}

LiCl-CsCl Crystals

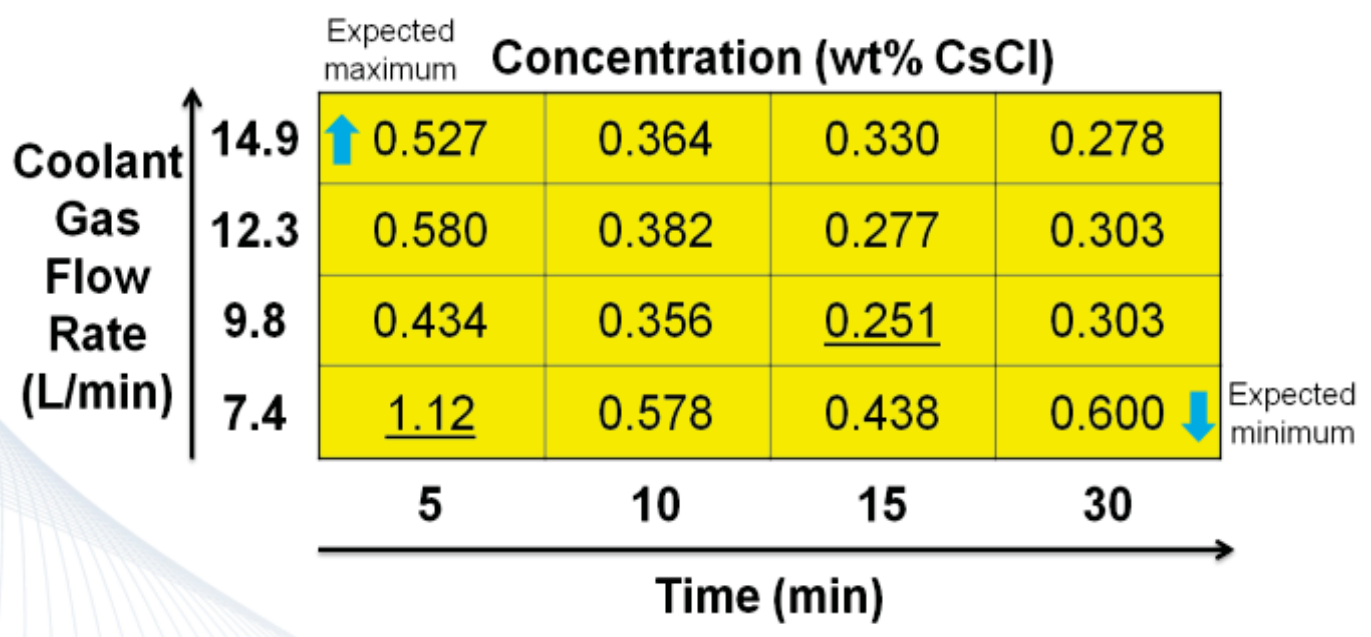

Note: LiCl-CsCl initially @ 5 wt $\% \mathrm{CsCl}$

\section{$\mathrm{LiCl}$ vs. LiCl-CsCl Crystals}

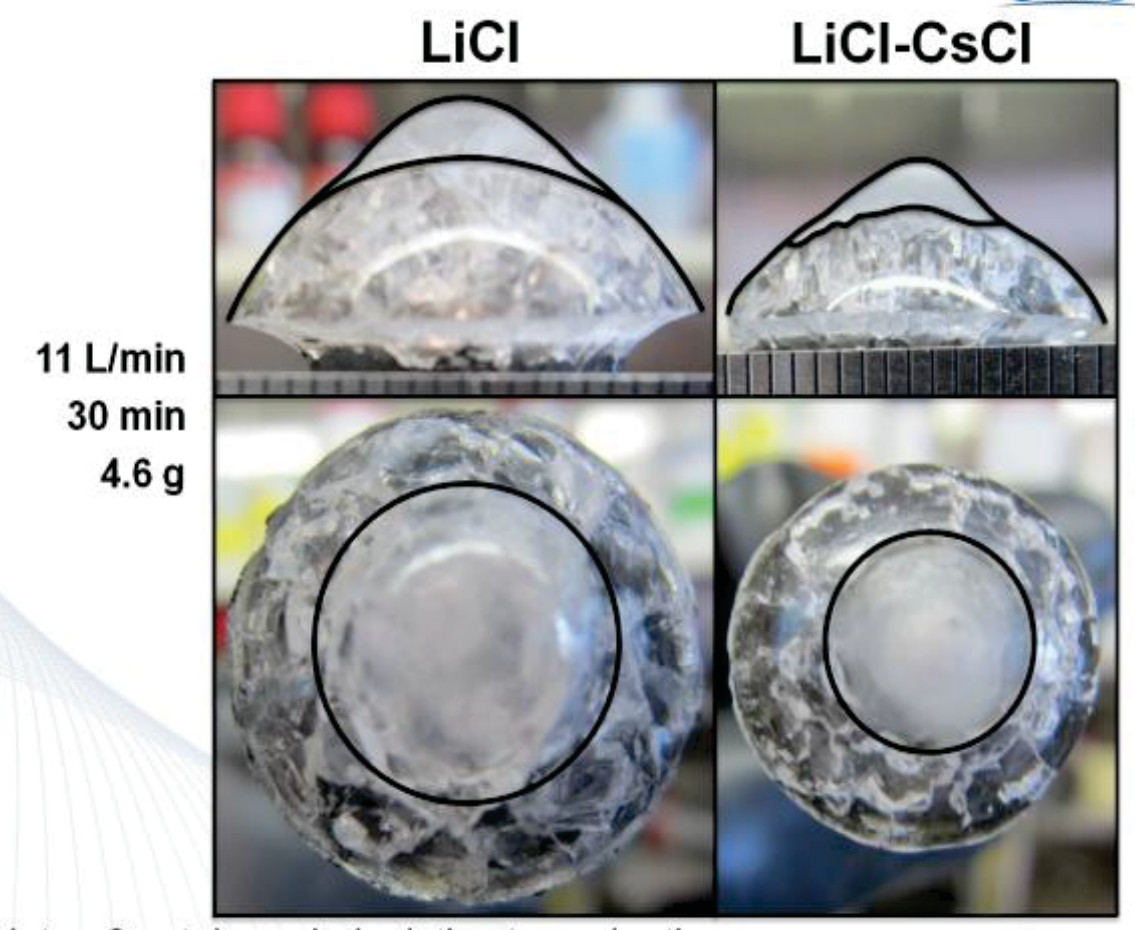




\section{Crystal Growth}

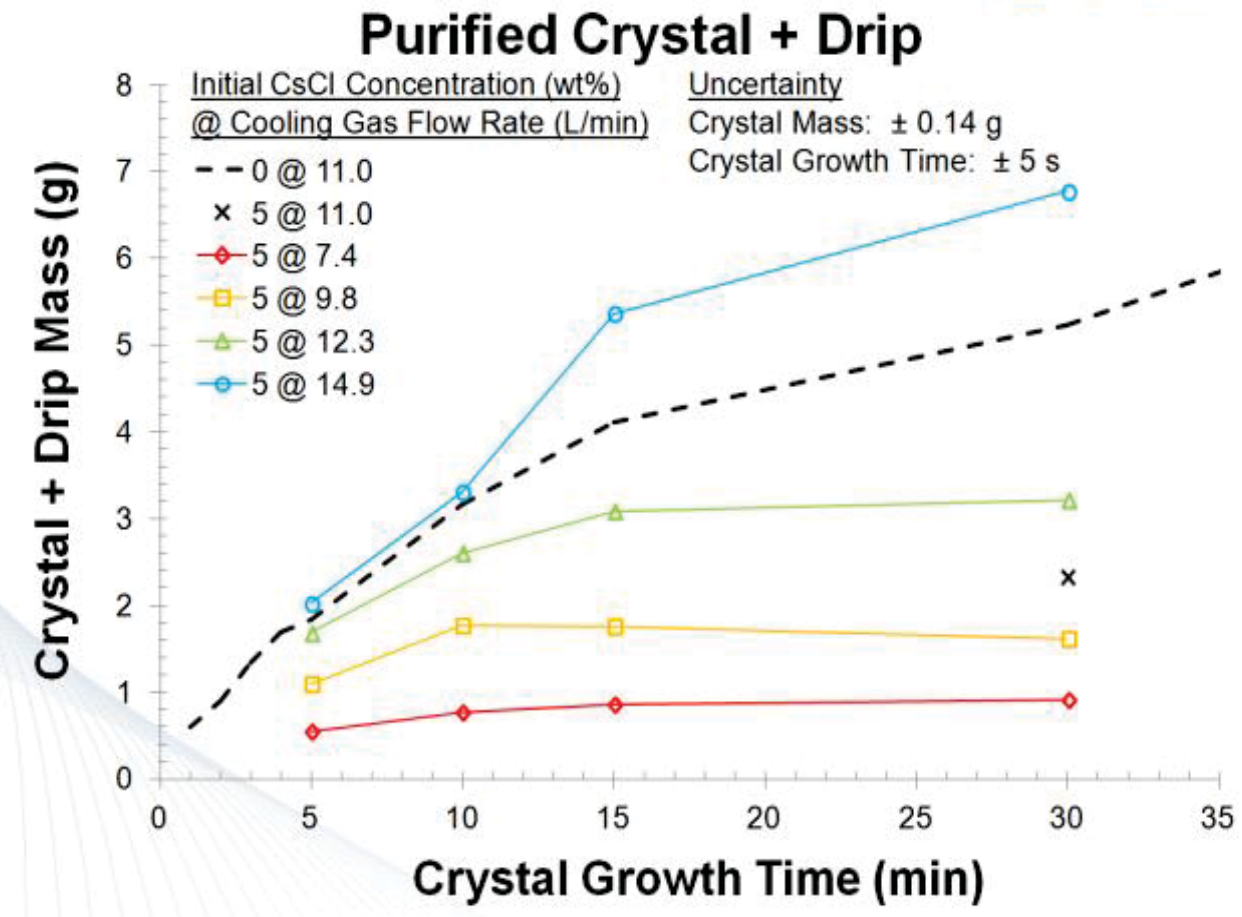

\section{Optimal Duration}

\section{Purified Crystal + Drip}

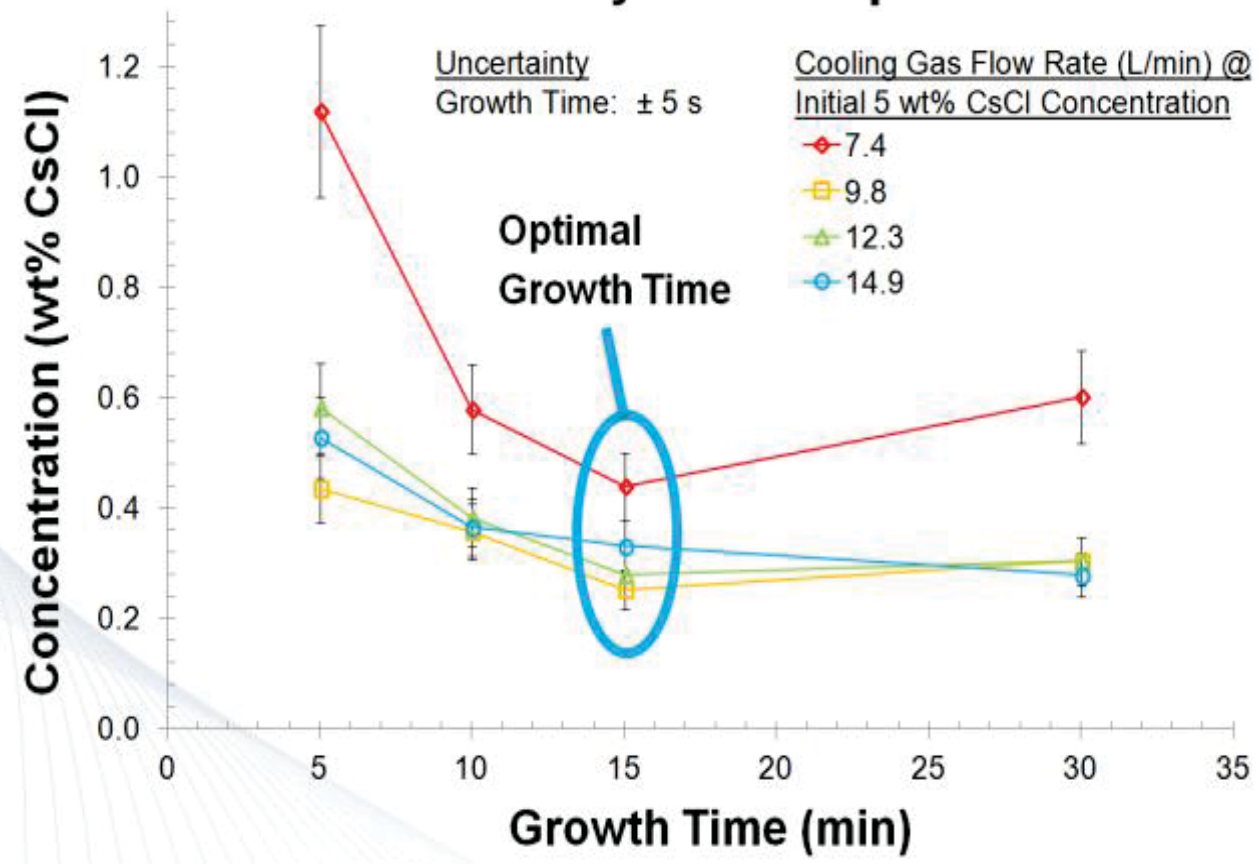




\section{Optimal Coolant Flow Rate}

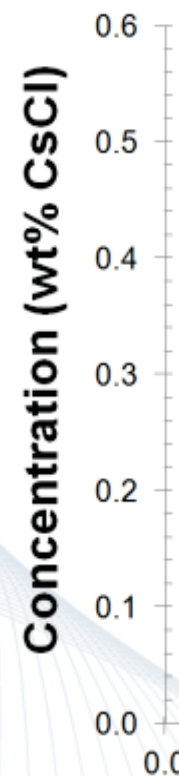

\section{Purified Crystal + Drip}

Cooling Gas Flow Rate (L/min)@

Initial $5 \mathrm{wt} \% \mathrm{CsCl}$ Concentration

and 15 min Growth Time

$\diamond 7.4$

$\square 9.8$

\begin{tabular}{l}
$\triangle 12.3$ \\
\hline 014.9
\end{tabular}
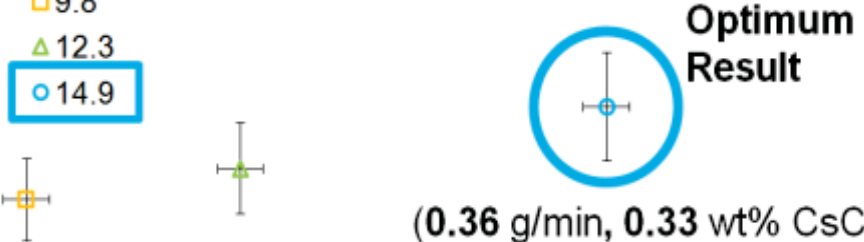

$(0.36 \mathrm{~g} / \mathrm{min}, 0.33 \mathrm{wt} \% \mathrm{CsCl})$

(0.12 g/min, 0.25 wt $\% \mathrm{CsCl})$

1

0.0

0.1

0.2

0.3

0.4

Growth Rate (g/min)

\section{Drip Concentration}

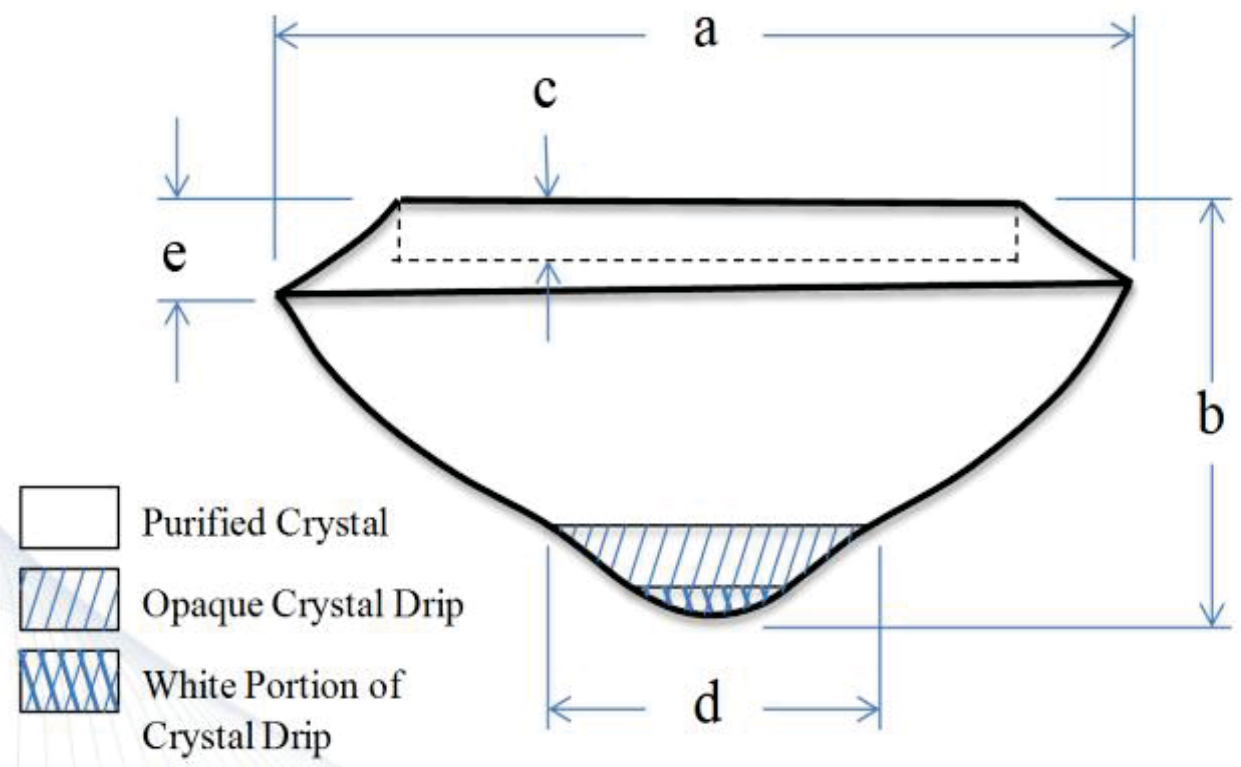




\section{Drip Concentration}

\section{Concentrated Drip vs. Bulk}

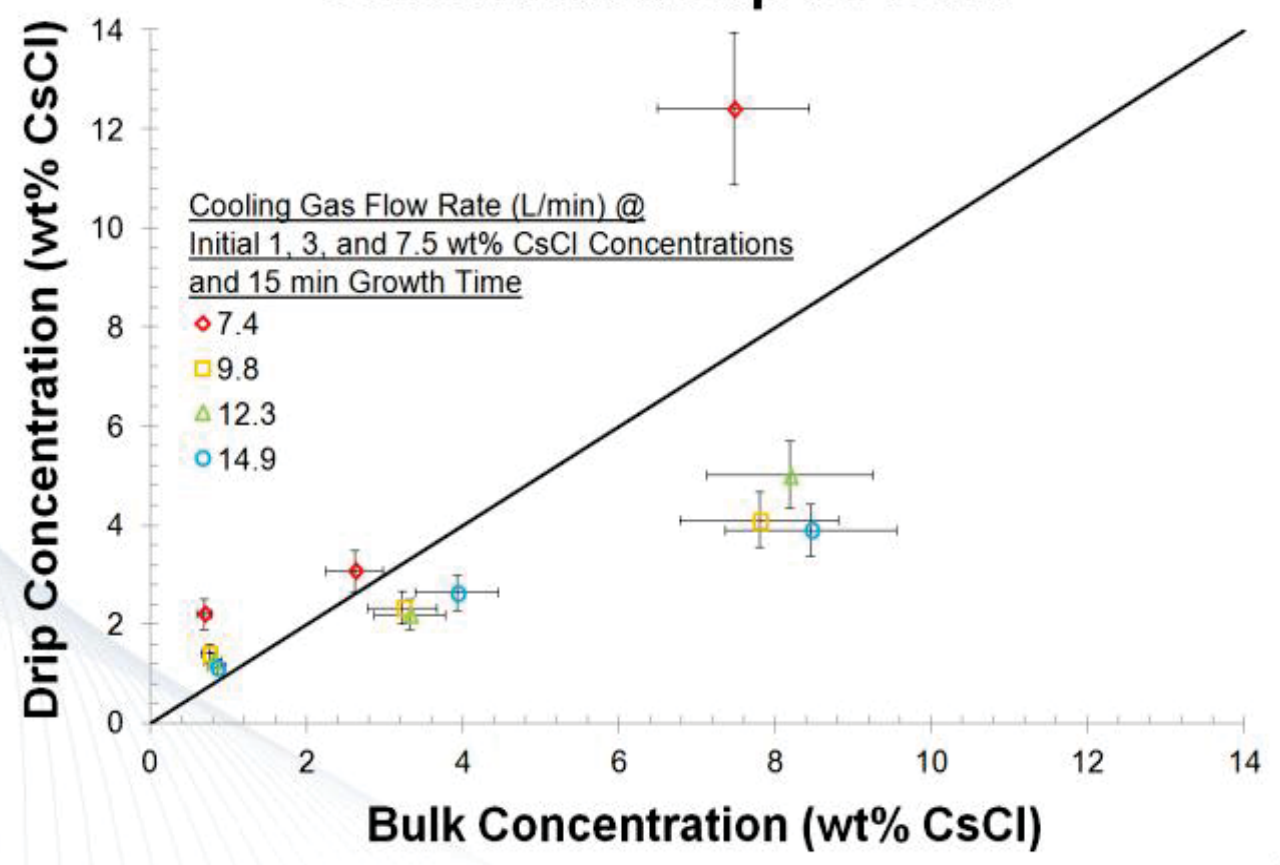

Initial Conc. Effect on Growth

Purified Crystal + Drip

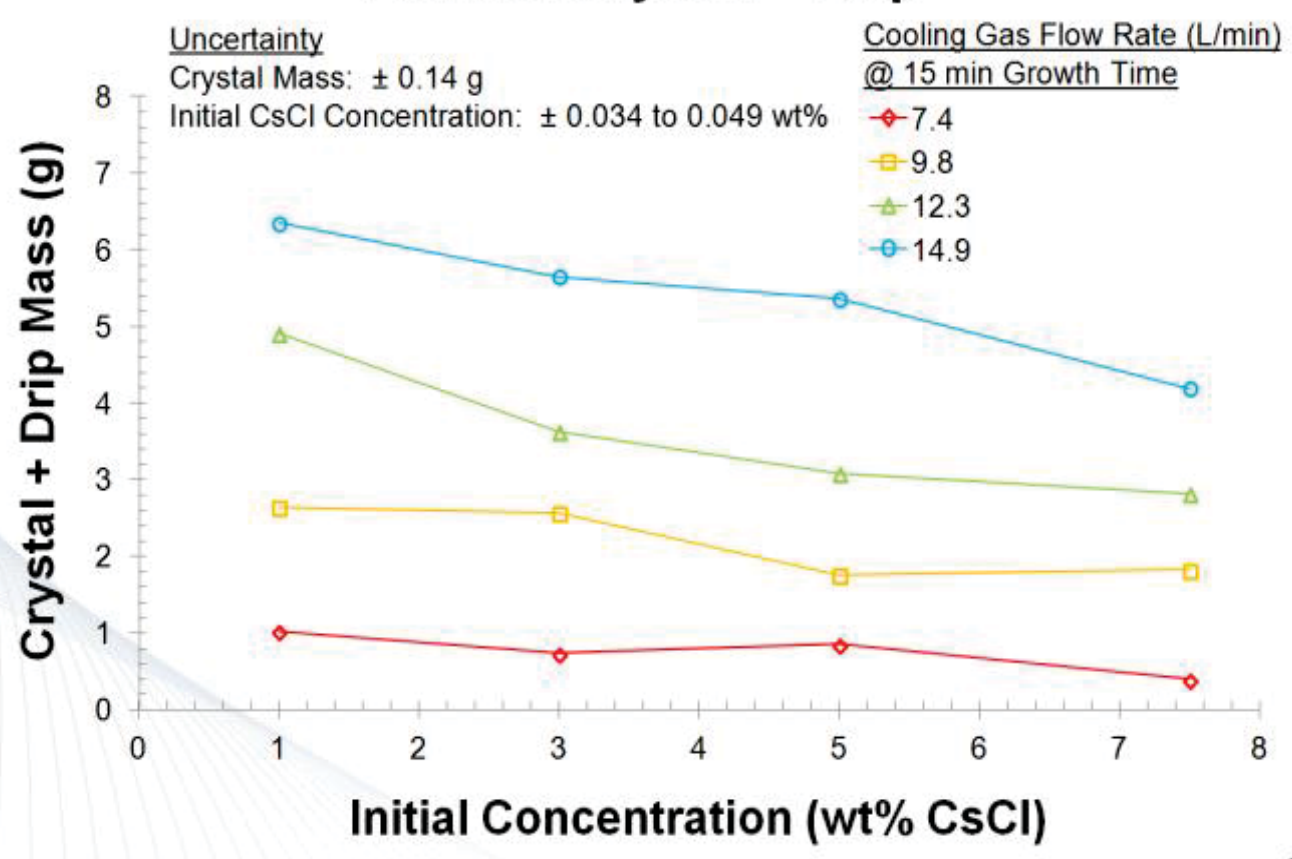



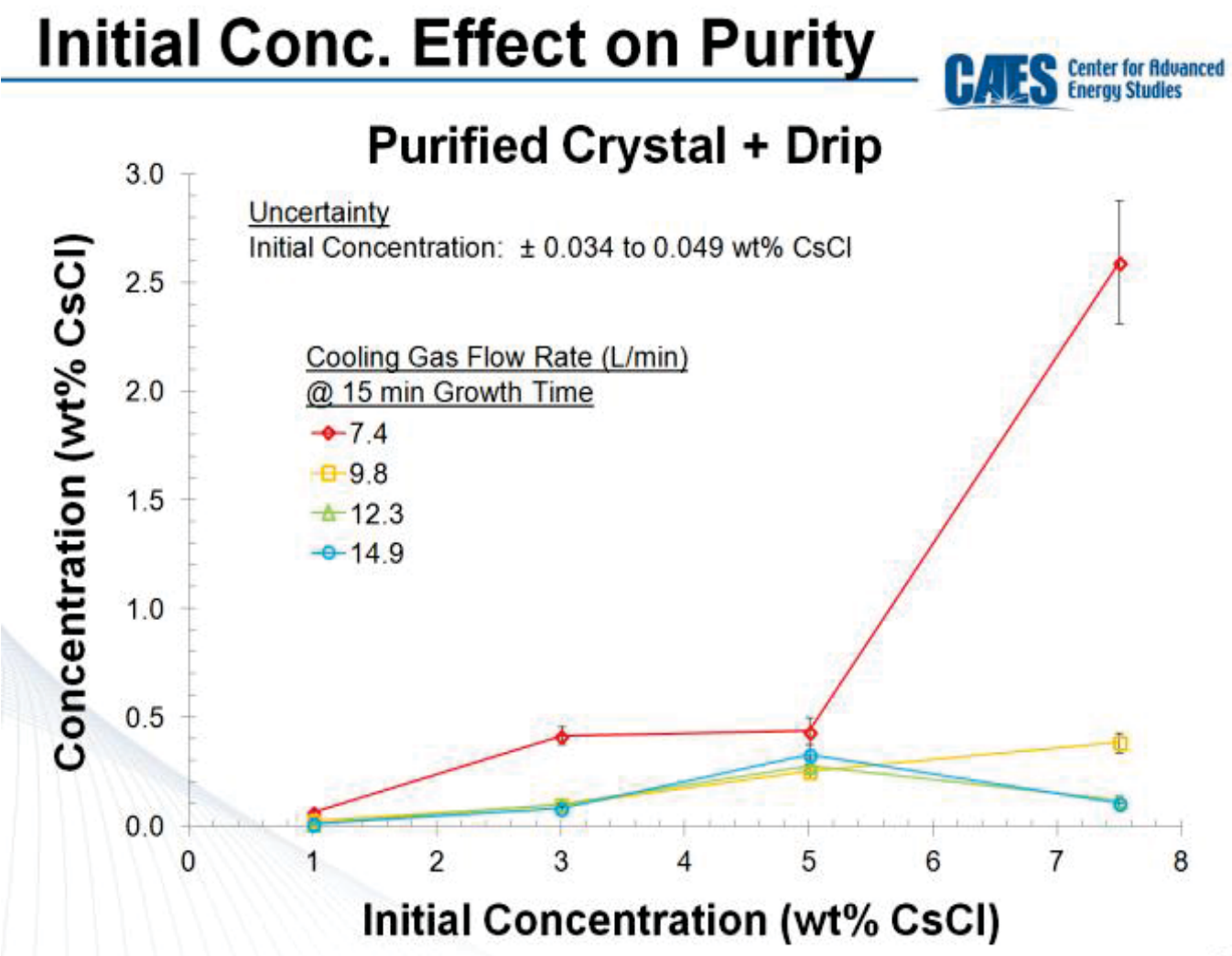

\section{Potential Separation}

\section{Separation Scenario:}

- Concentrate used electrolyte from 5 to $7.5 \mathrm{wt} \% \mathrm{CsCl}$

- With 7 cold fingers

- Operating at 15 min crystal growth time and

- $14.9 \mathrm{~L} / \mathrm{min}$ cooling gas flow rate

- Requiring 7 growth cycles and

- Total operation time of $105 \mathrm{~min}$

\section{Potential Results:}

- Production rate of $136 \mathrm{~g} / \mathrm{hr}$

- With purity of $0.24 \mathrm{wt} \% \mathrm{CsCl}$
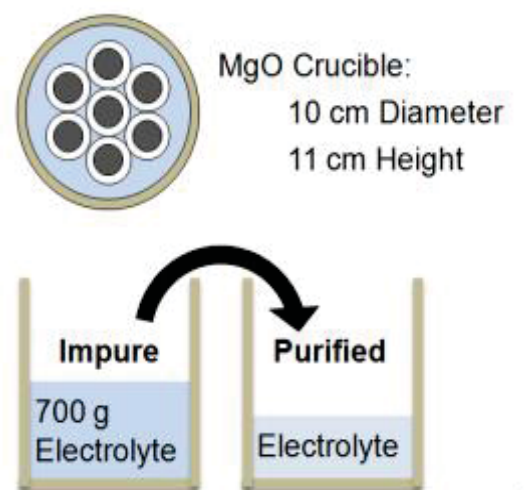


\section{Crystal Growth Correlations}

\begin{tabular}{llccccc}
\hline Effects & Correlation & $a$ & $b$ & $R$ & $R^{2}$ & $P_{C}$ \\
\hline $\begin{array}{l}\text { Heat } \\
\text { Transfer }\end{array}$ & $\eta=a(G r P r)^{b}$ & $1.41 \mathrm{E}-10$ & 1.624 & 0.925 & 0.855 & $3.85 \mathrm{E}-13$ \\
$\begin{array}{l}\text { Mass } \\
\begin{array}{l}\text { Transfer } \\
\text { Heat \& Mass }\end{array}\end{array}$ & $\eta=a(G r S c)^{b}$ & $1.26 \mathrm{E}-13$ & 1.586 & 0.930 & 0.864 & $1.64 \mathrm{E}-13$ \\
Transfers & $\eta=a(G r P r S c)^{b}$ & $2.52 \mathrm{E}-14$ & 1.565 & 0.932 & 0.868 & $1.05 \mathrm{E}-13$ \\
\hline
\end{tabular}

$$
\begin{gathered}
\eta=\frac{m}{m_{0}}=\frac{\text { Final Crystal mass }}{\text { Initial LiCl mass }} \quad \operatorname{Pr}=\frac{\mu c_{P}}{k}=\frac{\text { Viscous diffusion }}{\text { Thermal diffusion }} \\
G r=\frac{g \beta|\Delta T| L^{3} \rho^{2}}{\mu^{2}}=\frac{\text { Buoyancy force }}{\text { Viscous force }} \quad S c=\frac{\mu}{\rho D_{A B}}=\frac{\text { Viscous diffusion }}{\text { Species diffusion }} \\
P_{C}(R, N)=\frac{1}{\sqrt{\pi}} \frac{\Gamma\left(\frac{N-1}{2}\right)}{\Gamma\left(\frac{N-2}{2}\right)} \int_{|R|}^{1}\left(1-x^{2}\right)^{(N-4) / 2} d x
\end{gathered}
$$

\section{Nomenclature}

\begin{tabular}{lll}
\hline Symbol & Description & Unit \\
\hline$a \& b$ & Constants in correlations & none \\
$c_{P}$ & Specific heat capacity of molten salt & $\mathrm{J} /(\mathrm{kg} \mathrm{K})$ \\
$D_{A B}$ & Species diffusion coefficient & $\mathrm{m} / \mathrm{s}$ \\
$g$ & Gravitational constant & $\mathrm{m} / \mathrm{s}^{2}$ \\
$k$ & Thermal conductivity of molten salt & $\mathrm{W} /(\mathrm{m} \mathrm{K})$ \\
$L$ & Characteristic length $\left(L=\left(m / \rho_{s}\right)^{1 / 3}\right)$ & $\mathrm{m}$ \\
$m$ & Mass of purified crystal & $\mathrm{kg}$ \\
$m_{0}$ & Mass of initial LiCl in $30 \mathrm{~g}$ of $\mathrm{LiCl}-\mathrm{CsCl}$ & $\mathrm{kg}$ \\
$N$ & Number of uncorrelated experimental data points & none \\
$\beta$ & Thermal expansion coefficient & $\mathrm{K}-1$ \\
$\Gamma(k)$ & The Euler gamma function to the factorial function $\mathrm{k!}$ & $\mathrm{none}$ \\
$\Delta T$ & Temperature difference between bulk and solid/liquid & $\mathrm{K}$ \\
$\eta$ & interface & $\mathrm{none}$ \\
$\mu$ & Dimensionless purified crystal mass & $\mathrm{kg} /(\mathrm{s} \mathrm{m})$ \\
$\rho$ & Dynamic viscosity of molten salt & $\mathrm{kg} / \mathrm{m}^{3}$ \\
$\rho_{S}$ & Density of molten salt & $\mathrm{kg} / \mathrm{m}^{3}$ \\
\hline
\end{tabular}




\section{Crystal Growth Correlations}
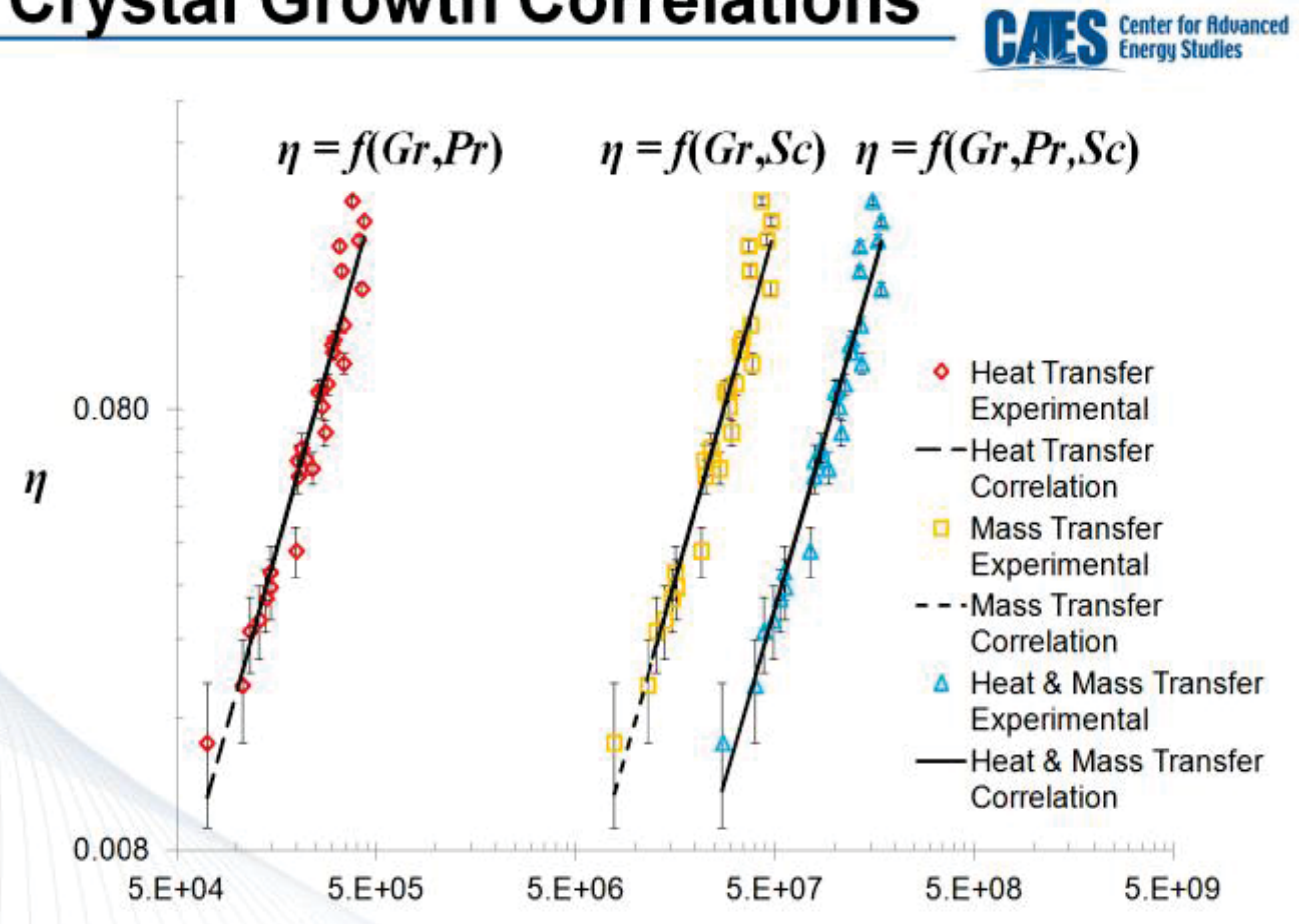

$(G r P r),(G r S c)$, and $(G r P r S c)$

\section{Crystal Growth Correlations}

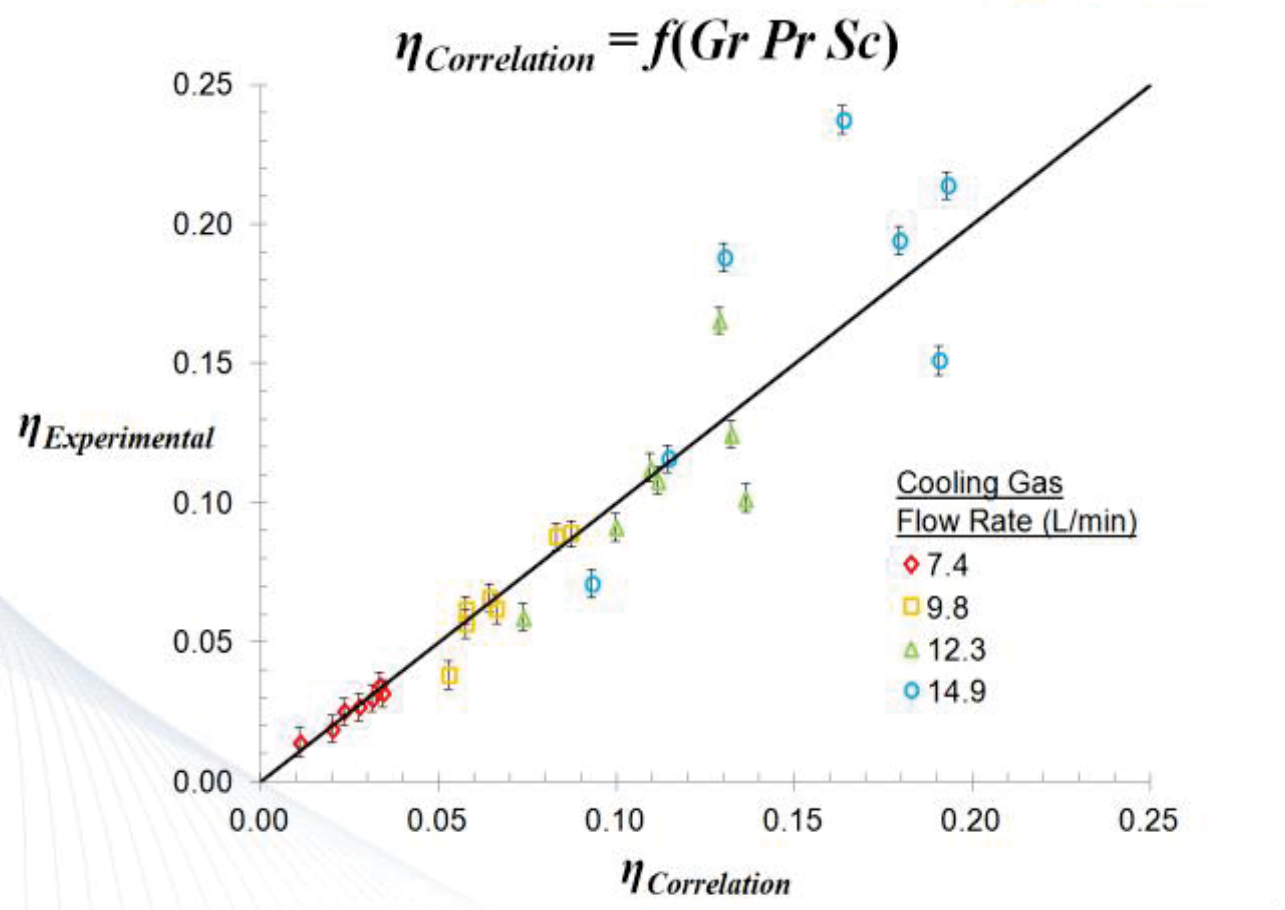




\section{Summary}

\section{Optimal Parameters}

" $14.9 \mathrm{~L} / \mathrm{min}$ (Cooling gas flow rate)

" 15 min (Crystal growth time)

\section{Optimal Results}

" $0.36 \mathrm{~g} / \mathrm{min}$ (Crystal growth rate)

- 0.33 wt $\% \mathrm{CsCl}$ (Crystal purity)

Potential Separation

- Concentrate electrolyte from 5 to $7.5 w t \% \mathrm{CsCl}$

- $136 \mathrm{~g} / \mathrm{hr}$ (Production rate)

- $0.24 w t \% \mathrm{CsCl}$ (Purity)

\section{Best Correlation}

- $\eta=2.52 \mathrm{E}-14\left(\operatorname{Gr} P r S_{c}\right)^{1.565}$

- $R^{2}=0.868$

- Cooling gas flow rate is the most controlling operating parameter

- Suggesting that heat transfer predominantly influences growth rate

\section{Next Step ...}

Further understand the separation process with:

\section{Experimental work}

- Validate potential scale up scenario results

" Explore salt systems with more fission products

- Strontium(Sr)

- Actual electrolytic oxide reduction electrolyte used to process commercial oxide fuel

\section{Literature review}

- Benchmark cold finger separation production and purity with all other processes, including

- Zone freezing and

- Drawdown techniques 


\section{Acknowledgements}

" Idaho National Laboratory's (INL)

- Laboratory Directed Research and Development (LDRD) program for funding

- Center for Advanced Energy Studies (CAES)

- for support of the LDRD funding and

- providing laboratory space (Radiochemistry Lab) for this project

- Major Professor

- Supathorn Phongikaroon

- Committee Members

- Akira Tokuhiro

- Vivek Utgikar

- Colleagues

- Michael Simpson, Michael Shaltry, Rob Hoover, Ammon Williams, Ryan Bezzant, Michael Pack, Dalsung Yoon, Cindy Hanson, and Debbie Lacroix

\section{Questions}

\section{LiCl-CsCl (Initial 5 wt\% CsCl)}

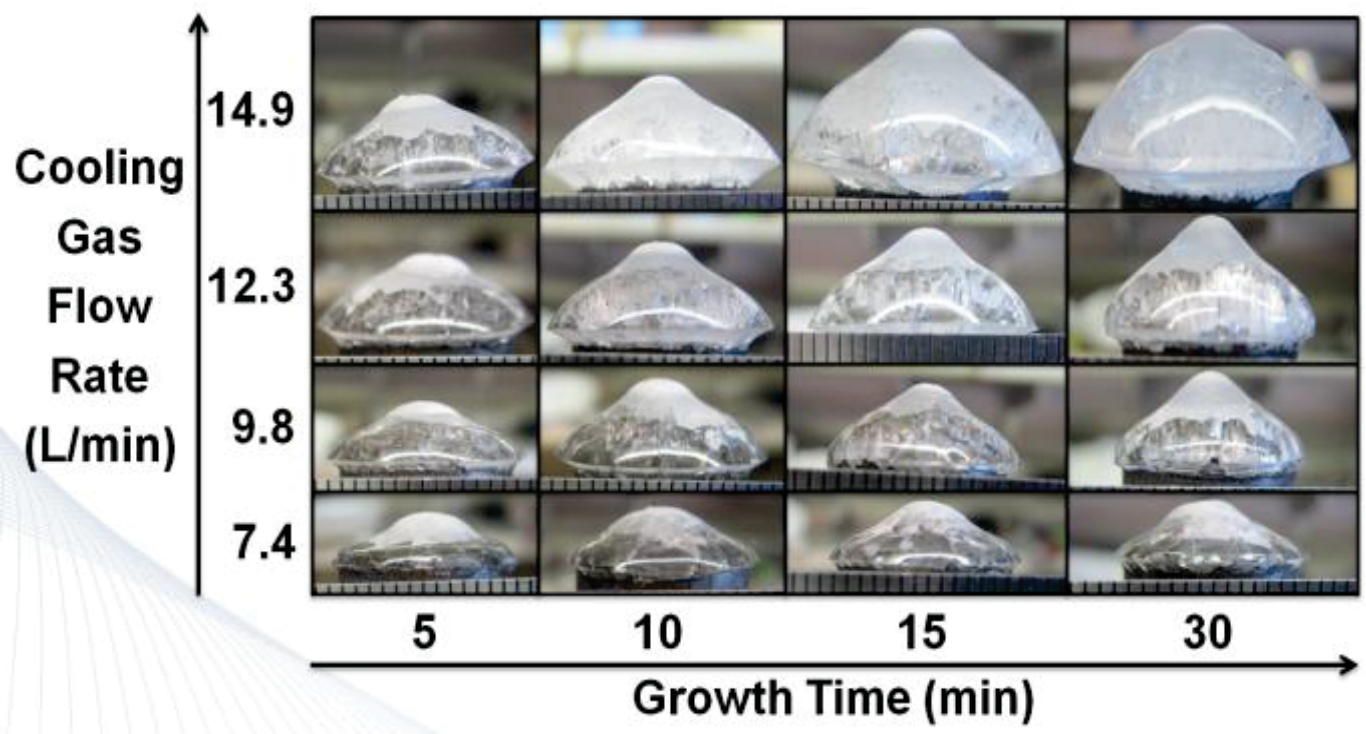




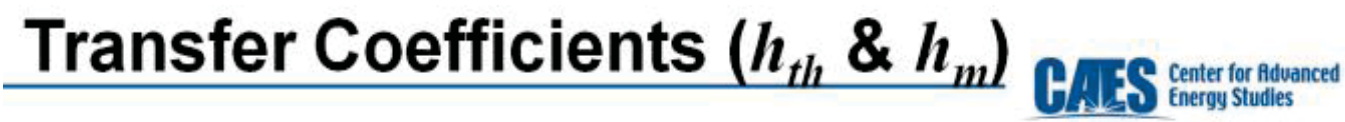

$$
\begin{aligned}
& N u=\frac{h_{t h} L}{k}=\frac{\text { Convection heat transfer }}{\text { Conduction heat transfer }} \quad S h=\frac{h_{m} L}{D_{A B}}=\frac{\text { Overall mass diffusion }}{\text { Species diffilsion }} \\
& N u \text { or } S h=a(G r \operatorname{Pr} S c)^{b} \quad L=\left(\frac{m}{\rho_{s}}\right)^{1 / 3} \quad \eta=N u^{3} \text { and } S h^{3}
\end{aligned}
$$

\begin{tabular}{lcccc}
\hline Effects & Correlation & Average $\eta$ & $\begin{array}{c}\text { Average } h_{t h} \\
\left(\mathrm{~W} / \mathrm{m}^{2} \mathrm{~K}\right)\end{array}$ & $\begin{array}{c}\text { Average } h_{m} \\
(\mathrm{~m} / \mathrm{s})\end{array}$ \\
\hline $\begin{array}{l}\text { Heat } \\
\text { Transfer }\end{array}$ & $\eta=a(\mathrm{Gr} \mathrm{Pr})^{b}$ & $0.0893 \pm 0.0520$ & $26.2 \pm 1.64$ & $1.02 \mathrm{E}-07 \pm 7.03 \mathrm{E}-09$ \\
$\begin{array}{l}\text { Mass } \\
\begin{array}{l}\text { Transfer } \\
\text { Heat \& }\end{array}\end{array}$ & $\eta=a(\mathrm{GrSc})^{b}$ & $0.0889 \pm 0.0520$ & $26.1 \pm 1.57$ & $1.02 \mathrm{E}-07 \pm 6.72 \mathrm{E}-09$ \\
$\begin{array}{l}\text { Mass } \\
\text { Transfers }\end{array}$ & $\eta=a(\mathrm{Gr} \mathrm{PrSc})^{b}$ & $0.0895 \pm 0.0524$ & $26.2 \pm 1.55$ & $1.02 \mathrm{E}-07 \pm 6.58 \mathrm{E}-09$ \\
\hline
\end{tabular}

$h_{t h}$ values for natural convective systems are reported to be within the range of $50-1000 \mathrm{~W} /\left(\mathrm{m}^{2} \mathrm{~K}\right)$ for liquids (Incropera).

\section{Dimensionless Magnitudes}

$$
\begin{array}{ll}
\eta=0.09=\frac{\text { Final Crystal mass }}{\text { Initial LiCl mass }} & P r=3.5=\frac{\text { Viscous diffi } \text { sion }}{\text { Thermal diffusion }} \\
G r=70,000=\frac{\text { Buoyancy force }}{\text { Viscous force }} & S c=390=\frac{\text { Viscous diffusion }}{\text { Species diffusion }}
\end{array}
$$

$$
\begin{aligned}
& \operatorname{Ra}=\operatorname{Gr} P r=2 \times 10^{\wedge} 5=\frac{\text { Buoyancy force }}{\text { Viscous force }} \\
& B i=\frac{h_{t h} L}{k_{s}}=5 x 10^{\wedge}-5=\frac{\text { Surface thermal resistance }}{\text { Internal thermal resistance }}
\end{aligned}
$$

